\title{
Polymer-mode-coupling theory of the slow dynamics of entangled macromolecular fluids
}

\author{
Kenneth S. Schweizer*, Matthias Fuchs ${ }^{\text {a) }}$, G. Szamel ${ }^{\text {b) }}$, Marina Guenza ${ }^{\text {c) }}$, Hai Tang \\ Departments of Materials Science \& Engineering, Chemistry, \\ and Materials Research Laboratory, University of Illinois, \\ 1304 W.Green Street, Urbana, IL 61801, U.S.A.
}

\section{SUMMARY:}

A microscopic statistical dynamical theory of the slow dynamics of entangled macromolecular fluids has been formulated at the level of effective generalized Langevin equations-of-motion of a tagged polymer. A novel macromolecular version of mode-coupling theory is employed to approximately capture the cooperative motions of entangled polymers induced by the long range, self-similiar interchain correlations. Polymer integral equation methods are used to determine the required equilibrium structural input. Entanglements arise due to time and space correlations of the excluded volume forces exerted by the surrounding matrix on a tagged macromolecule. A spatially resolved description of entanglement constraint amplitudes relates the fluctuating forces to fluid structure. Constraint relaxation proceeds via three parallel processes: probe center-of-mass translation and shape fluctuations, and collective matrix relaxation. Asymptotic scaling law predictions for the molecular weight and concentration dependences of transport coefficients and relaxation times of chain polymer solutions and melts are in qualitative agreement with the phenomenological reptation theory. Predictions for finite frequency properties such as anomalous diffusion, and shear stress and dielectric relaxation, are derived. Enhanced, power law dissipation for properties controlled by conformational relaxation is predicted, with the corresponding frequency scaling exponents in good agreement with experiments but differing from reptation behavior. For experimentally accessible chain lengths strong finite size corrections for the transport coefficients arise due to entanglement constraint porosity and constraint release. Successful quantitative applications to many experimental data sets suggest the theory provides a unified microscopic understanding of the non-asymptotic scaling laws observed for the viscosity, dielectric relaxation time, and solution self and tracer diffusion constants. Generalization to fractal macromolecular architectures allows semi-quantitative treatment of ring and spherical microgel melts, and tracer diffusion in gels. A theory for the influence of concentration fluctuations in entangled polymer blends and diblock copolymers has also been developed. Self-diffusion in blends is quantitatively suppressed due to dynamical constraints associated with domain formation. Much stronger suppression of diffusion and chain

a) Present address: Physik Department, Technische Universität München, James-Franck Straße, 85747 Garching, Germany.

b) Present address: Department of Chemistry, Colorado State University, Fort Collins, CO 80523, U.S.A.

c) Permanent address: Istituto di Studi Chimico-Fisici di Macromolecule, Sintetiche e Naturali, IMAG, CNR, Via de Marini 6, Genova, Italy. 
relaxation is predicted near and well below the order-disorder transition of diblock copolymer melts due to microdomain formation. New dynamical scaling laws are predicted, and quantitative agreement of the theory with recent measurements on polyolefin diblocks is demonstrated. Limitations of the theory, open problems, and possible future directions are discussed.

\section{Introduction}

The dynamics of solutions and melts composed of long chain polymers is very unusual due to the phenomenon of "entanglement"1-3). At the microscopic scale, entanglement arises as the dynamical consequences of chain connectivity and backbone uncrossability due to intermolecular repulsive excluded volume forces. A dramatic, nearly universal slowing down of long time conformational relaxation and transport processes occurs, and a rubber-like elastic response emerges over a wide intermediate time scale. New scaling laws for the degree of polymerization and concentration dependences of transport coefficients and relaxation times are found, along with anomalous short time translational diffusion and extended power law frequency dependences of stress, dielectric, and other dynamic response functions. Crossovers between early time/small displacement (short chain) "unentangled" dynamics, and longer time/larger displacement (long chain) entangled dynamics occurs in a manner which depends on nonuniversal features of molecular structure, polymer density, and the specific dynamic property of interest. Distinctive nonlinear rheological phenomena also emerge ${ }^{1-3)}$.

The entanglement phenomenon is not unique to long chain polymers. It also occurs for many different macromolecular architectures ${ }^{3-5)}$, such as cyclic rings ${ }^{6}$, star-branched polymers ${ }^{4,7)}$, rigid rods ${ }^{3)}$, comb and $\mathrm{H}$-like molecules ${ }^{8)}$, and even melts of non-interpenetrating microgel particles" or "rubber balls". The key requirement is simply a sufficiently dense collection of mutually repelling macromolecules, most often with a large number of internal degrees of freedom ("flexibility"). Many qualitative aspects of entangled dynamics, particularly at long times, depend on the global macromolecular architecture. However, short time features (e.g., rubbery plateau modulus), and some particular systems for all times (e.g., ring and chain melts ${ }^{6)}$ ), exhibit common, nearly architecture-independent behavior. Slow dynamics also occurs in more complex fluids such as polyelectrolytes ${ }^{10}$, where excluded volume and Coulombic forces are both present, and polymer blends ${ }^{11)}$, self-assembling block copolymers ${ }^{12}$ ) and associating polymers ${ }^{13)}$ (e.g., ionomers) where long range concentration fluctuations and (micro)domain formation can result in a strong coupling of thermodynamic and entanglement constraints.

The traditional qualitative interpretation of the physical origin of entanglement phenomena is that a transient network emerges due to "topological interactions" amongst the long and interpenetrating random coils ${ }^{1-3)}$. The transient network structure severely retards the conformational relaxation of individual chains. The slowing down phenomena are viewed as of purely dynamical origin, not related to any equilibrium structural constraints. Many phenomenological theories have been constructed for the linear chain case based on strikingly different ansatzes for real space motion $^{1-5,14-19)}$. The elegant reptation/tube theory of deGennes ${ }^{2)}$, and its further 
quantification and extension by Doi and Edwards ${ }^{3)}$, is the most developed and widely applied approach. It focuses on the motion of a single chain in an effectively static field of "topological constraints" due to the surrounding polymers, the "tube". A chain disentangles by a stochastic sliding motion. The hallmark of the tube model is the postulate of a type of dynamic localization into a reduced space (the static random walk tube), with escape via coherent anisotropic diffusion along its own (coarse-grained) contour. Many qualitative predictions of the simplest version of reptation theory are in good, or reasonable, accord with experiment ${ }^{5)}$. The description of several of the nonlinear rheological phenomena, and generalization of the tube ideas to entangled suspensions of rods and branched macromolecules, are particularly noteworthy and useful ${ }^{3-5}$. Although widely accepted, there remain controversies about the literal veracity of the reptation idea and its elaborations ${ }^{5,14)}$. It is not our purpose here to focus on such issues, but several are worth mentioning.

There are significant discrepancies between simple or "pure" reptation model predictions and experiments on chain fluids ${ }^{3,5)}$. For example, the experimental shear viscosity ${ }^{1)}$ and melt dielectric relaxation time ${ }^{20)}$ scale with degree of polymerization, $N$, as $N^{y}$ with $y=3.5 \pm 0.2$, self and tracer diffusion in solutions and gels scale ${ }^{5,21)}$ as $D \sim N^{-x}$ with $x=2.3-3$, the pre-terminal frequency dependence of the dielectric ${ }^{20 \text { ) }}$ and stress ${ }^{1,22)}$ loss moduli do not agree with each other and are both much broader than the pure reptation prediction, and the surprising similiarity of viscoelastic properties of melts of linear chains, (non-reptating) polymer rings, and/or non-fractal microgels (rubber balls) ${ }^{6,9)}$. Many attempts over the past 20 years have been made to construct "extended" reptation/tube models which incorporate alternative nonreptative motions based on guesses about the meaning of entanglements and the confining tube ${ }^{3-5)}$. These include processes known as "contour length fluctuations" 3,23 , "reptons" ${ }^{24)}$, "double reptation" ${ }^{25)}$, "constraint release"4), "tube dilation/plastization" "26), and "nematic or orientational coupling" 27). All such mechanisms are viewed as corrections to the basic reptation picture, and usually serve to weaken the rigid tube constraint and enhance relaxation for chains of non-infinite length. Each phenomenological idea has been formulated in multiple manners by different workers, with empirical parameters of sometimes vague microscopic meaning which enter as adjustable constants for fitting data. The question of whether such elaborations are physically reasonable, and have "explained" the behaviors not properly described by pure reptation, is a subjective matter. The similarity of the viscoelasticity of melts of chains, rings, and microgels appears particularly difficult to reconcile within a reptation framework ${ }^{9)}$.

Pure reptation is a highly nontrivial statement at the level of the $N$-body dynamics of a tagged chain. Despite several attempts ${ }^{28-30}$, it has never been derived from fundamental principles, and hence it is difficult to a priori know its range of validity or limitations. Moreover, due to its highly phenomenological character, descriptions of entanglement at the level of the time-dependent intermolecular forces cannot be addressed. Moreover, prediction of the influence of liquid structure on dynamics is not attainable or requires extra assumptions (e.g., reptation + scaling ansatz for solutions $\mathrm{s}^{2,3)}$ ). Computer simulations can at present only treat lightly entangled fluids which are inadequate for definitively testing the reptation hypothesis at the chain 
trajectory level ${ }^{14,31)}$. Clever experiments based on birefringence, dichroism, or neutron reflectivity have attempted to detect anisotropic motions of labeled chains $^{5,32,33)}$. Unfortunately, since both isotropic and anisotropic motions produce measurable signals, conclusions concerning the importance of reptative motion involve quantitative data analysis and model fitting, and are thereby difficult to unambiguously interpret ${ }^{32-34)}$. Visual microscopy experiments ${ }^{35)}$ in solution are also subject to a number of potentially complicating features.

The recent interesting phenomenological approaches by Herman ${ }^{17)}$, and Douglas and Hubbard ${ }^{18)}$, emphasize the importance of many chain, non-reptative cooperative isotropic motions. They challenge the common assumption that the dominant contribution to stress is of single chain origin. The Douglas and Hubbard theory is built on the concept of "dynamically correlated motion of clusters of chains". Novel predictions are made for the dependence of transport coefficients and response functions on polymer fractal and spatial dimensions ${ }^{18)}$.

Over the past eight years, we have developed, and widely applied, a novel microscopic statistical dynamical theory of entangled macromolecular fluids ${ }^{36-48}$. A long term goal is to provide a unified approach to treat all the systems described above within a common theoretical framework. By "microscopic", we mean constructing a theory formulated at the level of segmental intermolecular forces from which observable dynamical properties can be deduced. This philosophy stands in strong contrast to essentially all other phenomenological attempts to describe entangled dynamics based on ansatzes about chain motion and topological constraints. A guiding (but speculative) idea is that entanglement effects may emerge as a natural consequence of the unique equilibrium structural correlations of fluids composed of macromolecules, such as the self-similiar, architecture-dependent intra- and intermolecular correlations on intermediate length scales. In particular, intermolecular pair correlations must possess a "correlation hole" of spatial range of order macromolecular size due to the combined constraints of polymer connectivity and intermolecular excluded volume forces ${ }^{2,49}$. As is done for other slow dynamics problems $^{50)}$ such as caging ${ }^{51)}$ and glass formation ${ }^{52)}$ in colloidal and atomic fluids, critical phenomena ${ }^{53,54)}$, diffusion in disordered media and fracton dynamics ${ }^{55}$, and some aspects of biopolymer dynamics ${ }^{56}$, the goal is to approximately compute the dynamical consequences of structural constraints as encoded in ensemble-averaged fluctuating force time correlations, or memory functions, which describe the statistically-averaged influence of many body caging on the motion of single macromolecules.

Major theoretical progress in describing the equilibrium structure of macromolecular fluids has occurred over the past decade based on generalizations of nonperturbative integral equation methods ${ }^{57)}$. The most developed and widely applied approach is the "Polymer Reference Interaction Site Model" (PRISM) theory of Curro and Schweizer ${ }^{57)}$, which is based on the small molecule RISM theory of Chandler and Andersen ${ }^{58)}$. A prediction of PRISM theory for the intermolecular carbon-carbon radial distribution function in a polyethylene melt ${ }^{59)}$ is shown in Fig. 1. As in any dense liquid, there is system-specific structure on local scales. However, strong solvation shells are not present due to the inherent thermal conformational 
disorder of polymers and multiple local chemical length scales which frustrate the layering behavior commonly seen in dense simple fluids. The local structure persists out to $3-4 \mathrm{CH}_{2}$ group diameters $(\cong 15 \AA)$ in a nearly molecular weight independent fashion. However, when the chains get "long enough", a power law correlation hole regime emerges, $h(r)=g(r)-1 \sim-r^{-1}$, which extends over a broad intermediate regime which widens as the chain radius of gyration increases, $R_{\mathrm{g}} \sim N^{1 / 2}$. The number of correlated matrix polymer segments on a spherical shell of radius $r$ centered on a tagged monomer scales as $-4 \pi r^{2} h(r)$, which grows linearly with $r$ on intermediate length scales.

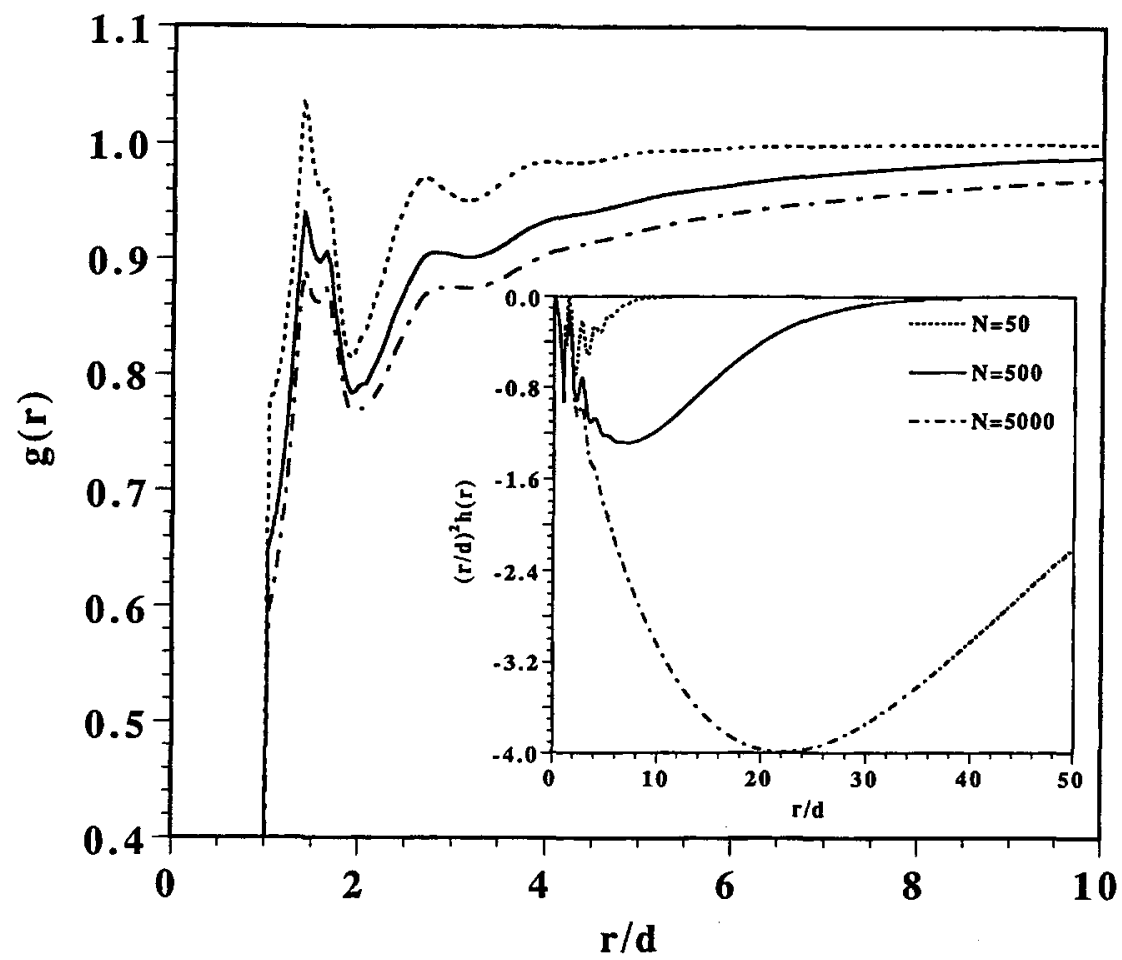

Fig. 1. PRISM theory intermolecular carbon-carbon pair correlation function, $g(r)$, as a function of reduced separation for a hard core (site diameter $d=3.9 \AA$ ) model polyethylene melt at $430 \mathrm{~K}$ and $N=50$ (short-dash), 500 (solid), and 5000 (dash-dot). $R_{\mathrm{g}} / d \cong 2.6$, 9.2 , and 29.6, respectively. The inset shows the curves for $r^{2}(g(r)-1)=r^{2} h(r)$

Geometrically, ideal polymer chains are low density fractal objects in the sense that the single coil density is of order $N / R_{\mathrm{g}}^{3} \sim N^{-1 / 2}$. This feature implies copious interpenetration, or "entanglement", of $\cong N^{1 / 2}$ different chains in a 3-dimensional spherical volume of radius $R_{\mathrm{g}}$. There also exists an of order $N^{1 / 2}$ spatially correlated "contacts" between a pair of entangled chains. Based on the known monomer volume and chain statistics of polyethylene, these geometric features imply roughly 100 mutually entangled chains in a high molecular weight melt of $N=10000$. 
The general behavior of the numerical results in Fig. 1 are qualitatively well described by the analytic predictions of PRISM theory based on a simplified Gaussian thread model of polymer chains ${ }^{57,60)}$ :

$$
g(r)=1+\frac{3}{\pi \rho \sigma^{2} r}\left(\exp \left(-r / \xi_{\rho}\right)-\exp \left(-r / \xi_{\mathrm{c}}\right)\right)
$$

Here, $\xi_{\rho} \sim\left(\rho \sigma^{2}\right)^{-1}$ is the (nonuniversal) density fluctuation screening length, $\rho$ is the segmental number density, $\sigma$ is the statistical segment length, and $\xi_{\mathrm{c}}=R_{\mathrm{g}} / 2^{1 / 2}$ is the universal correlation hole length scale. This behavior of $g(r)$ is analogous to a fluid near a critical point where local packing is unaffected as the correlation length diverges $\left(\xi_{\mathrm{c}} \rightarrow \infty\right.$ as $\left.N \rightarrow \infty\right)$. The obvious difference is that an entangled polymer melt is not near a thermodynamic critical point since the collective density fluctuations are noncritical and very small. However, entanglement is a phenomenon associated with the excluded volume forces between segments on different chains. Thus, $g(r)$ is the relevant structural property required to describe interchain forces. On rather general physical grounds one might then expect that such long range spatial correlations would induce highly cooperative motions which could be the origin of slow entangled dynamics. The unentangled-entangled crossover degree of polymerization, $N_{\mathrm{e}}$, would roughly correspond to when the correlation hole regime in $g(r)$ emerges. Alternatively, for long highly entangled chains the crossover length scale $r_{\mathrm{c}}$ (or a time scale $\tau_{\mathrm{c}}$ ) would roughly correspond to when segments have been displaced a distance where the self-similiar interchain packing correlations emerge. Strong renormalization of the $N$-dependences of the conformational relaxation time and transport coefficients would be associated with the correlation hole length $\xi_{\mathrm{c}} \sim$ $N^{1 / 2} \sigma$, while intermediate time anomalous diffusion and power law stress and dielectric relaxation would emerge due to the self-similiar packing which controls the fluctuating interchain forces in the fluid.

The qualitative physical scenario sketched above is what the polymer mode-coupling (PMC) theory attempts to mathematically capture ${ }^{36)}$. It is based on the presumption of isotropic, correlated many segment motions which are the dynamical consequence of the spatially long range intermolecular correlations between interpenetrating, fractal-like macromolecules in a dense fluid state. Such a viewpoint is in strong contrast with the purely dynamical "topological entanglement" perspective ${ }^{2,3)}$. However, it has much in common with our modern understanding of other slow dynamics problems such as critical phenomena, fracton dynamics, caging in simple fluids, and the glass transition ${ }^{50-55)}$. It is important to realize that mode-coupling theories have led to a microscopic understanding of several fluid dynamical problems (e.g., the latter two) where both structure and dynamics are dominated by hard core repulsive forces.

At a technical level, mathematical tractability of PMC theory requires significant "pre-averaging" approximations. In particular, an isotropic cage-averaged description is employed to describe a single macromolecule embedded in a dense polymer fluid. $N$ coupled, nonlocal, and non-Markovian linear generalized Langevin equations (GLE) are derived to describe the motion of a tagged or probe macromolecule influenced by entanglement caging effects. Linearity of the GLE's ensures analytic 
tractability, and corresponds to the assumption that the Rouse normal modes ${ }^{3)}$ remain "good" even in entangled fluids. Thus, PMC theory may be viewed as a form of harmonic effective medium theory which may, or may not, be a literally true description of individual macromolecule trajectories. Isotropy-enforcing pre-averaging does not a priori preclude the theory from describing the experimentally measurable ensemble-averaged properties of entangled isotropic polymer fluids. However, prediction of some physical properties might be compromised by a cage-averaged theory, and the ability to describe reptation in the anisotropic motion sense is not possible within such a theoretical framework. Recent work by Kawasaki ${ }^{29)}$ has attempted to combine the PMC approach with reptation concepts as formulated by Hess $^{28)}$. However, this work remains at a tentative, preliminary stage. We suspect chain motion in real entangled chain fluids involves significant isotropic and anisotropic components, so pure reptation and PMC theory perhaps represent limiting descriptions.

This feature article has multiple goals. (i) Present an overview of the fundamental physical and mathematical content of PMC theory, including the underlying assumptions and approximations. (ii) Discuss the key qualitative analytic predictions in the limit of high molecular weight. (iii) Describe progress in quantitative applications to experiments, including analytic and numerical treatments of the very important finite size corrections. (iv) Clearly identify the similarities and differences of PMC and reptation/tube theories. (v) Provide suggestions for future experiments and/or simulations to probe untested predictions of the theory.

PMC theory is mathematically demanding, involving techniques unfamiliar to most polymer scientists and many physicists. Moreover, it represents a very unconventional approach to the entanglement problem. Here, we choose to present the key results largely without derivation, but provide new intuitive arguments and interpretations of the physical content of PMC theory. We hope such a presentation style will make PMC theory more accessible to the polymer community, particularly experimentalists. The detailed mathematical analysis can be found in the original papers $^{36-48)}$, along with a description of equilibrium PRISM theory ${ }^{57)}$. The presentation will largely, but not completely, follow the historical development.

The remainder of the paper is structured as follows. The general formulation of PMC theory for linear chain homopolymer solutions and melts, and the qualitative predictions for the coupled GLE's and memory functions, is given in section II. Large $N$, asymptotic scaling predictions for chain melts and solutions are discussed in section III. Section IV describes the incorporation of the very important finite $N$ fluctuation corrections in the theory at a simplified, physically-transparent analytic level. Model calculations, and detailed quantitative applications to experimental data, are also presented. Generalization to treat tracer diffusion in gels and networks is described in section V. A numerical version of PMC theory, which approximately treats finite $N$ corrections and the unentangled-entangled crossover, is described in section VI. Generalization of PMC theory to entangled fluids composed of interpenetrating fractal architectures is the subject of section VII. Section VIII describes recent generalizations to treat the coupling of thermodynamic and entanglement constraints in homopolymer blends and self-assembling diblock copolymer melts. 
The paper concludes in section IX with a discussion of present limitations of the theory, open problems, and possible future directions.

\section{General formulation of a statistical dynamical theory}

In the spirit of the classic Rouse, Zimm, and reptation theories ${ }^{1-3)}$, the PMC theory focuses on deriving effective equations-of-motions for a single tagged polymer dissolved in a macromolecular fluid matrix. Consider first the simplest case of a one-component homopolymer fluid composed of linear chain polymers of identical elementary units or "sites". This case is relevant to melts, and also solutions under the standard simplification of representing the solvent molecules as a vacuum or increased free volume. Theta and good solvent conditions can be treated, but hydrodynamic interactions are assumed fully screened and thus ignored ${ }^{2,3)}$.

The polymers are modeled as a linear sequence of interaction sites, which in the most general case interact intramolecularly and intermolecularly via site-site pair potentials of arbitrary form. Rigid constraints, such as a fixed nearest neighbor bond length, are not considered. The general theory described below could be taken as the starting point to treat local polymer dynamics. However, for the entanglement problem we shall adopt the standard coarse-grained Gaussian bead-spring chain model $^{3)}$. Very local dynamics (inside a segment scale $\sigma$ ) are treated as irreversible and characterized by a friction constant, $\zeta_{0}$, with white noise fluctuating forces in the Rouse model ${ }^{3)}$ spirit.

\section{A. Projection operator analysis and GLE's}

The most general approach employs a phase space projection operator scheme within a Mori-Zwanzig framework ${ }^{50)}$ to derive effective generalized Langevin equations (GLE) for the $N$ tagged polymer segments ${ }^{36)}$. Since this projection is based on the most general single molecule $\mathrm{N}$-body field, intramolecular and intermolecular forces are rigorously separated. Further simplification occurs by making the standard Brownian overdamped assumption that inertial effects are irrelevant and segment velocites relax very rapidly compared to the segment displacements $\left\{\boldsymbol{r}_{\alpha}(t)\right\}, \alpha$ $=1,2, \ldots . N$. The resulting $N$ coupled GLE's represent a non-Markovian set of force balance equations ${ }^{36)}$

$$
\begin{aligned}
& 0=-\frac{\partial W[\{\boldsymbol{r}(t)\}]}{\mathrm{\partial} \boldsymbol{r}_{a}(t)}-\zeta_{0} \frac{\mathrm{d} \boldsymbol{r}_{a}(t)}{\mathrm{d} t}-\sum_{\gamma=1}^{N} \int_{0}^{t} \mathrm{~d} \tau \Gamma_{a \gamma}(t-\tau ;\{\boldsymbol{r}(\tau)\}) \cdot \frac{\mathrm{d} \boldsymbol{r}_{\gamma}(\tau)}{\mathrm{d} \tau} \\
&+\boldsymbol{f}_{a}(t)+\boldsymbol{F}_{a}^{Q}(t) \\
& \Gamma_{a \gamma}^{i j}(t-\tau) \equiv \beta\left\langle F_{a}^{i}(0) F_{\gamma}^{j}(t-\tau) \prod_{\gamma=1}^{N} \delta\left(\boldsymbol{r}_{\lambda}(t)-\boldsymbol{r}_{\lambda}^{Q}(t-\tau)\right)\right\rangle
\end{aligned}
$$

Here, $W[\{r(t)\}]$ is the many body potential of mean force which includes the bare intrachain pair interactions plus the condensed-phase-induced component, $\zeta_{0}$ is the "bare" friction constant describing irreversible frictional processes on the micro- 
scopic scale and $f_{a}(t)$ is the corresponding white noise random force, $F_{a}^{Q}(t)$ is the projected fluctuating "random force" on the segmental scale where, schematically, $Q=1-P$ with $P$ a projection operator onto the most general $N$-body single polymer field, and $\Gamma_{a \gamma}^{i j}$ is a tensorial matrix of segmental scale intermolecular fluctuating forces which evolve via the projected time evolution operator. The latter quantity plays the role of a memory function tensor, and contains all many body information about entanglement forces. The product of constraining delta functions implies the memory function is also a functional of the entire $N$-body polymer trajectory, in both real and projected dynamical space. The effective caging forces at time $t$ require dynamical knowledge at all earlier times. The exact GLE's are both nonMarkovian and extremely nonlinear, and thus are entirely intractable.

We believe that the proper description of an anisotropic reptative type motion requires retaining the tensorial, $N$-body nature of the memory function. At present, tractable approximations to achieve this are unknown. Thus, a "pre-averaging", or mean field cage, approximation was adopted by Schweizer corresponding to factorizing the forces and constraining delta functions in Eq. (2.1). Physically, this means the dependence of the memory function on the instantaneous $\mathrm{N}$-body trajectory of the tagged polymer is ignored, which rigorously results in a set of GLE's of isotropic form $^{36)}$

$$
\begin{aligned}
& 0=-\frac{\partial W[\{\boldsymbol{r}(t)\}]}{\partial \boldsymbol{r}_{a}(t)}-\zeta_{0} \frac{\mathrm{d} \boldsymbol{r}_{a}(t)}{\mathrm{d} t}-\sum_{\gamma=1}^{N} \int_{0}^{t} \mathrm{~d} \tau \Gamma_{a \gamma}(t-\tau) \cdot \frac{\mathrm{d} \boldsymbol{r}_{\gamma}(\tau)}{\mathrm{d} \tau} \\
&+\boldsymbol{f}_{a}(t)+\boldsymbol{F}_{a}^{Q}(t) \\
& \Gamma_{a \gamma}(t) \equiv(\beta / 3)\left\langle\boldsymbol{F}_{\alpha}(0) \cdot \boldsymbol{F}_{\gamma}^{Q}(t)\right\rangle
\end{aligned}
$$

where $\beta=\left(k_{\mathrm{B}} T\right)^{-1}$ is the inverse thermal energy. The memory function is now a diagonal tensor, and is not a functional of the instantaneous polymer trajectory. However, $\Gamma_{a \gamma}(t)$ is an $N \times N$ matrix which describes the ensembled-averaged statistical correlation between the total force exerted at $t=0$ by all the matrix polymers on tagged polymer segment $\alpha$ with the corresponding total force exerted on tagged polymer segment $\gamma$ at a later time $t$. Thus, it still contains the full (intractable) many body physics.

Eq. (2.2) remains nonlinear if the potential of mean force is not harmonic. However, adopting the standard Flory ideality idea yields a quadratic form, $W=(3 / 2)$ $\beta^{-1} \sigma^{-2} \Sigma_{a}\left(\boldsymbol{r}_{a}-\boldsymbol{r}_{a+1}\right)^{2}$. Eq. (2.2) then becomes

$$
\zeta_{0} \frac{\partial \boldsymbol{r}_{a}(t)}{\partial t}+\int_{0}^{N} \mathrm{~d} \gamma \int_{0}^{t} \mathrm{~d} \tau \Gamma_{a \gamma}(t-\tau) \frac{\partial \boldsymbol{r}_{\gamma}(\tau)}{\partial \tau}=K_{\mathrm{S}} \frac{\partial^{2} \boldsymbol{r}_{a}(t)}{\partial a^{2}}+\boldsymbol{f}_{a}(t)+\boldsymbol{F}_{a}^{Q}(t)
$$

where $K_{\mathrm{S}}=3 \beta^{-1} \sigma^{-2}$ is the entropic spring constant connecting statistical segments, and the standard continuum description of the chain has been adopted ${ }^{3)}$. Formally, Eq. (2.4) is of a linear, non-Markovian, generalized Rouse form with the bare fric- 
tion constant replaced by a time-dependent matrix of fluctuating force correlation functions $\Gamma_{a \gamma}(\mathrm{t})$. Nonlinearity could still be implicitly present if the memory function is treated self-consistently, i.e. as a functional of ensemble-averaged correlations involving segmental coordinates evolving via the true dynamical evolution operator. However, we shall not consider such a case in this paper. Thus, the Rouse normal $\operatorname{modes}^{3)}, r_{p}=N^{-1} \int_{0}^{N} \mathrm{~d} a \cos (p \pi a / N) r_{a}$ with $p=0,1,2, \ldots . . N$, are employed, which describe cooperative polymer motions of a characteristic wavelength $(N / p)^{1 / 2} \sigma$. They remain "good" modes in the sense the GLE's are diagonalized by them if chain end effects are ignored.

$$
\begin{aligned}
& \zeta_{0} \frac{\mathrm{d} r_{p}(t)}{\mathrm{d} t}+\int_{0}^{t} \mathrm{~d} \tau \Gamma_{p}(t-\tau) \frac{\mathrm{d} \boldsymbol{r}_{p}(\tau)}{\mathrm{d} \tau}+K_{\mathrm{S}}\left(\frac{p \pi}{N}\right)^{2} r_{p}(t)=f_{p}(t)+F_{p}^{\mathrm{R}}(t) \\
& \Gamma_{p}(t) \equiv \int_{0}^{N} \mathrm{~d} \lambda \cos (p \pi \lambda / N) \Gamma(\lambda \equiv|a-\gamma|, t)
\end{aligned}
$$

The Rouse mode diagonalization yields exact results in the long chain limit (effectively periodic boundary conditions are invoked $)^{36,47)}$. Eqs. $(2.4)-(2.6)$ are also directly derivable based on the tagged polymer positions and velocities as the primary slow variables, although the definition of the projected fluctuating random forces would be formally modified ${ }^{36,61)}$. From a technical point of view, use of a linear GLE approach seems nearly unavoidable for the high polymer problem due to the analytic intractability of handling $N$ coupled, nonlinear, non-Markovian (colored noise) stochastic differential equations.

The cage-averaging type approximation, and attendant isotropic dynamics description, precludes using Eqs. (2.4)-(2.6) to describe a symmetry-broken anisotropic reptative type motion. However, the latter can only be discussed for the motion of a single tagged chain based on a single set of initial conditions. Any amount of averaging must destroy anisotropy. That is, averaging over the initial conditions of a specific tagged polymer, or averaging over all chains in the bulk sample, will restore isotropy. The latter situation is what is generally relevant for experiments.

The generalized Rouse form also implies a stochastic Gaussian process description. This feature may restrict the theory from properly describing nonlinear and/or spatially-resolved time correlation functions if the true dynamics is not wellapproximated by a Gaussian process. However, linear time correlation functions of experimental interest should be much less sensitive to the Gaussian nature of the theory. These are expressable in terms of the normal mode time correlation functions, $C_{p}(t)=\left\langle r_{p}(0) \cdot r_{p}(t)\right\rangle$, which follow from Laplace transformation (variable $z$ ) of Eqs. (2.5) as

$$
C_{p}(z)=C_{p}(0)\left\{z+K_{\mathrm{S}} \frac{(p \pi / N)^{2}}{\zeta_{0}+\Gamma_{p}(z)}\right\}^{-1}, \quad C_{p}(0)=N \sigma^{2} /\left(6 \pi^{2} p^{2}\right)
$$

We note that effective Gaussian type GLE theories are common in many areas of condensed matter chemistry and physics such as isomerization and chemical reac- 
tion dynamics, solvation dynamics, vibrational relaxation, and tunneling problems ${ }^{62)}$. For the entangled polymer problem, a Gaussian type approach is obviously an uncontrolled approximation, but one might hope it is useful since the tagged polymer interacts simultaneously with a very large number of other polymer chains, of order $N^{1 / 2}$ for ideal coils. Thus, central limit theorem arguments could be invoked, as they often are for chemical dynamics problems involving dipolar forces where the solute interacts with a large number of solvent molecules. Alternatively, for the problem of electronic states in disordered materials where the wavefunctions are strongly localized, progress can be made for (experimentally observable) statistically-averaged properties using effective medium type theories based on a delocalized state description and frequency-dependent self-energies ${ }^{62)}$.

An advantage of the linear GLE description of Eq. (2.4) is that it naturally reduces to the well known Rouse theory for sufficiently small $N$ and/or polymer density. For future reference, we recall some key results of the Rouse theory corresponding to $F_{\alpha}^{Q}(t)=\Gamma_{a \gamma}(t)=0$ in Eq. (2.4). The simple Langevin equations of motion are ${ }^{3)}$

$$
\zeta_{0} \frac{\mathrm{d} r_{\alpha}(t)}{\mathrm{d} t}=K_{\mathrm{S}} \frac{\partial^{2} r_{a}(t)}{\partial \alpha^{2}}+f_{a}(t), \quad\left\langle f_{a}(0) \cdot f_{y}(t)\right\rangle=6 \beta^{-1} \zeta_{0} \delta_{a, y} \delta(t)
$$

Zero frequency properties such as the self-diffusion constant, $D$, the shear viscosity (presumed of intramolecular stress origin), $\eta$, the relaxation time of normal mode $p$, $\tau_{p}$, and the longest relaxation or Rouse time, $\tau_{\mathrm{R}}$, are

$$
\begin{aligned}
& D=\left(\beta \zeta_{0} N\right)^{-1}, \quad \eta=\left(\rho \sigma^{2} \zeta_{0} / 36\right) N \\
& \tau_{\mathrm{R}}=\beta \zeta_{0} \sigma^{2} N^{2} /\left(3 \pi^{2}\right) \equiv N^{2} \tau_{0}, \quad \tau_{p}=\tau_{\mathrm{R}} p^{-2}
\end{aligned}
$$

In contrast to atomic, molecular or colloidal fluids, Rouse theory assumes interchain contributions to the stress are negligible relative to the single polymer contributions. On intermediate time scales, $\tau_{0} \ll t \ll \tau_{\mathrm{R}}$, the intrachain stress relaxation modulus $G(t)$, and segmental mean square displacement, follow fractional power laws, while the center-of-mass motion is Fickian

$$
G(t) \sim t^{-1 / 2}, \quad\left\langle\left(\boldsymbol{r}_{a}-\boldsymbol{r}_{a}(t)\right)^{2}\right\rangle \sim \sigma^{2} \sqrt{t / \tau_{0}}, \quad\left\langle\left(\boldsymbol{R}_{\mathrm{cm}}-\boldsymbol{R}_{\mathrm{cm}}(t)\right)^{2}\right\rangle=6 D t
$$

Rouse theory describes rather well experiments and computer simulations in short chain unentangled melts. It also is generally assumed to apply at short times/displacements in entangled polymer fluids ${ }^{1-3)}$, although systematic deviations have been experimentally documented ${ }^{1,22)}$.

As a simple chain model, pair decomposable hard core interactions between segments on different (Gaussian) polymers are adopted. For simplicity, the hard core diameter, $d$, is set equal to the statistical segment length, $\sigma$, which in general depends on temperature and polymer density in solutions. This coarse-grained model is not expected to yield quantitative predictions in an a priori absolute magnitude sense. However, it should be capable of predicting relative chemical trends, polymer concentration dependences, and dependences on polymer architecture, $N$, time, etc. Further progress requires the development of an approximate theory of the memory 
function matrix, $\Gamma_{a \gamma}(t)$, describing segmental scale excluded volume force time correlations. $\Gamma_{a y}(t)$ involves 4-body static and dynamic correlations (2 segments each on the tagged chain and matrix polymers), and predictions for all observable properties follow from it. We describe first an oversimplified model based on dynamic perturbation theory.

\section{B. Renormalized Rouse theory and entanglement onset}

The Renormalized Rouse (RR) approach developed by Schweizer adopts the simplest possible approximation for the dynamics of the memory function, and works directly at the level of the bare excluded volume forces ${ }^{36}$. Based on a superposition factorization of 4-body time correlation functions, and employing a Fourier representation in terms of a wavevector variable $k$, one obtains ${ }^{36,42)}$

$$
\Gamma_{a \gamma}(t)=\frac{8}{3} \beta^{-1} \rho \sigma^{4} g^{2}(\sigma) \int_{0}^{\infty} \mathrm{d} k k^{2} j_{1}^{2}(k \sigma) \omega_{a \gamma}(k) S(k) F_{a \gamma}^{Q}(k, t) F_{\mathrm{coll}}^{Q}(k, t)
$$

Here, $g(\sigma)$ is the intermolecular pair correlation, or radial distribution, function evaluated at the contact distance where the hard core forces act, $S(k)=\omega(k)+\rho h(k)$ is the collective liquid structure factor, $\omega_{\alpha \gamma}(k)=\exp \left(-k^{2} \sigma^{2}|\alpha-\gamma| / 6\right)$ is the partial intrachain structure factor for segments $a$ and $\gamma, \omega(k)=N^{-1} \Sigma_{a \gamma} \omega_{a \gamma}(k)$ is the single chain structure factor (normalized so that $\omega(k)=N$ ), $\rho$ is the number density of polymer segments, and $j_{1}(x)$ is the first spherical Bessel function. Time-dependent information enters via normalized dynamic structure factors, or propagators, evolving via projected dynamics: the intrachain partial dynamic structure factor $F_{a \gamma}^{Q}(k, t)$ and its collective analog $F_{\text {coll }}^{Q}(k, t)$. Eq. (2.11) provides a direct connection between the memory functions and equilibrium structure. The integration over wavevector can be interpreted as the contribution associated with dynamical processes on a length scale $2 \pi / k$, which decays in a parallel fashion via single polymer and collective liquid motions.

A schematic illustration of the force correlations, and the length scales which enter the memory function, is given in Fig. 2 . The strength of the contact excluded volume forces exerted on the two tagged polymer segments $\alpha$ and $\gamma$ are quantified by the factors of $g(\sigma)$, and these forces are spatially correlated by the intrachain and collective correlation functions $\omega_{a y}$ and $S$, respectively. The density fluctuation screening length $\xi_{\rho}$ is short in a dense fluid, thereby requiring all 4 interacting segments to be spatially close at $t=0$. Such a 4-body configuration represents an "entanglement" in the theory, and is spatially realized for tagged polymer sites widely separated along the chain due to the connectivity correlations of random walks and the $\cong N^{1 / 2}$ "long range self-contacts" in 3-dimensions.

Explicit predictions require approximations for the dynamic propagators. The RR theory adopts the simplest approach of employing Rouse dynamics. This is in the spirit of a short time, or perturbative, approach at the level of the memory functions, and is a rough analog of the Enskog theory of simple fluids based on independent binary collisions ${ }^{36,50)}$. For the single chain propagator, a simplification is invoked: $F_{a \gamma}(k, t) \cong F^{\mathrm{R}}(k, t)=$ coherent single chain propagator. This approximation is accu- 


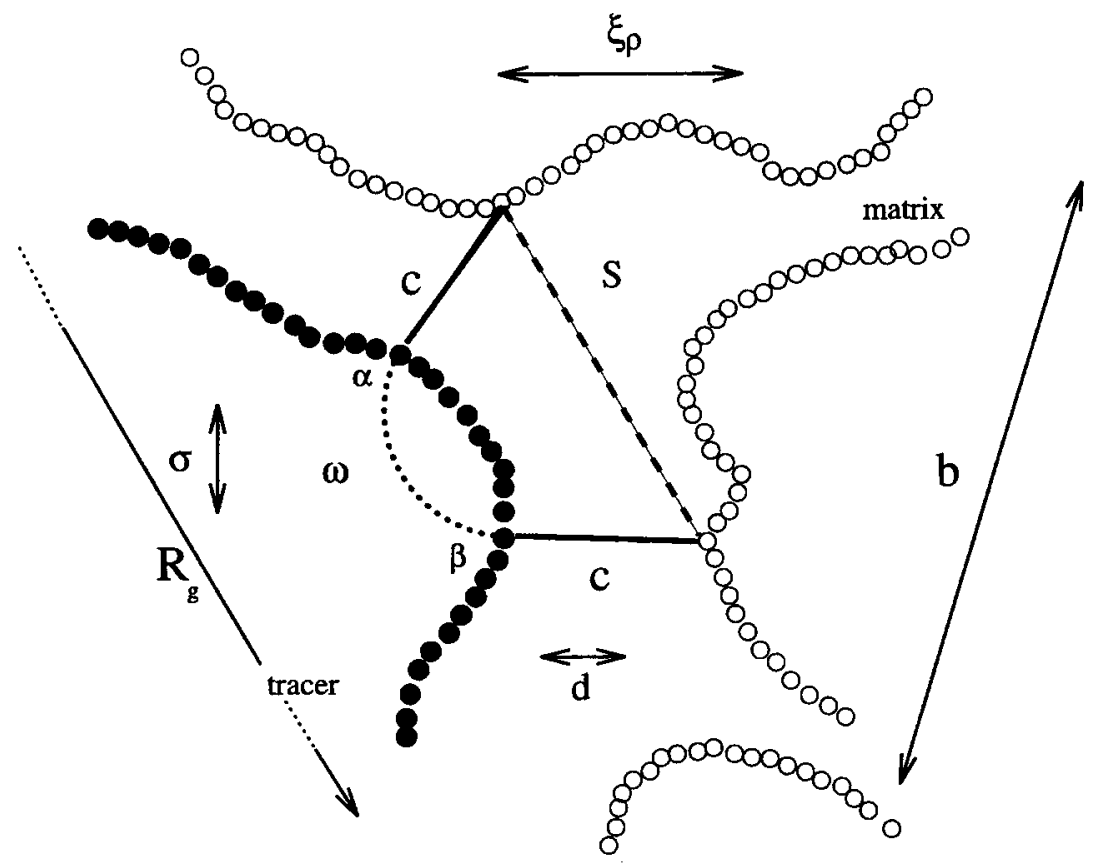

Fig. 2. Schematic illustration of the force correlations exerted by the matrix polymers on a tagged tracer chain, and the characteristic length scales which influence the entanglement friction memory functions. Two tracer segments $\alpha$ and $\beta$, spatially correlated by $\omega$ up to the tracer size, $R_{\mathrm{g}}$, interact via excluded volume pseudopotentials or direct correlations, $C$, with two matrix segments which are spatially correlated via the collective density fluctuation structure factor $S$. The system-specific, $N$-independent length scales are: segment length, $\sigma$, hard core diameter, $d$, the screening or mesh length, $\xi_{\rho}$, and the dynamic entanglement length, $b \equiv r_{\mathrm{c}}$

rate for intermediate and long time/length scales ${ }^{3)}$. In practice, the dynamic Random Phase Approximation expression is employed ${ }^{63)}$

$$
F^{\mathrm{R}}(k, t) \cong \exp \left[-k^{2} t /\left(\beta \zeta_{0} \omega(k)\right)\right]
$$

This form is adequate since it correctly describes the relation between $k$ and $t$ in the intermediate (segmental displacement dominated) and Fickian (center-of-mass displacement dominated) regimes. There are several possible approximations for the collective propagator ${ }^{50-53)}$ : (i) literal short time approximation, (ii) Vineyard-type approximation $F_{\text {coll }} \cong F^{\mathrm{R}}$, or (iii) an effectively "frozen matrix" approximation of $F_{\text {coll }} \cong 1$. Experience in simple liquids suggests the Vineyard approximation reliably describes, in a qualitative sense, time/space collective correlations ("caging") on molecular scales, although it fails in the hydrodynamic regime ${ }^{50)}$. For the entangled polymer problem, $R_{\mathrm{g}}$ and the dynamical crossover length scale $r_{\mathrm{c}}$ are the relevant 
"molecular" lengths associated with macromolecular caging, not the $k R_{\mathrm{g}} \ll 1$ hydrodynamic scales. For the case where the tagged and matrix polymers have identical degrees of polymerization, approximations (ii) and (iii) lead to qualitatively identical behavior when employed in Eq. (2.11). Thus, we adopt the "frozen matrix" approximation for simplicity, corresponding to the assumption that the fluctuating excluded volume force time correlations relax via collective tagged polymer Rouse motions.

Employing Eqs. (2.11) and (2.12) in Eqs. (2.5) and (2.6) results in an approximate theory for the time and $p$-mode dependent friction. As discussed elsewhere, the $p$ dependence of the memory function does not play a major role in determining most of the predictions of RR theory ${ }^{36,42)}$. Thus, for simplicity the $p$-dependence is ignored here, and RR theory reduces in form to a Rouse theory with an additional non-Markovian frictional force

$$
\Gamma_{\alpha \gamma}(t) \cong \delta_{a, \gamma} N^{-1} \sum_{\alpha, \gamma=1}^{N} \Gamma_{\alpha \gamma}(t) \equiv \delta_{a, \gamma} \Delta \zeta(t)
$$

where $\Delta \zeta(t)$ is given by Eq. (2.11) with the replacement $\omega_{a \gamma}(k) \rightarrow \omega(k)$. Interestingly, this time dependent memory function displays a regime of fractional power law decay: $\Delta \zeta(t) \sim \zeta_{0} \psi\left(\tau_{0} / t\right)^{-3 / 4}$ for intermediate time scales $\tau_{\mathrm{c}} \ll t \ll \tau_{\mathrm{R}}$, and hence modifies the Rouse dynamics on intermediate length scales $\sigma \ll r \ll R_{\mathrm{g}} . \tau_{\mathrm{c}}$ is a crossover time from bare Rouse to RR dynamics which is discussed below. The strength of the additional friction enters via the dimensionless structural parameter $\psi=$ $\rho \sigma^{3} g^{2}(d) S(0)$, which is proportional to the segmental scale mean square excluded volume force exerted by the matrix polymers on a tagged polymer segment. The fractional power law, or time fractal, behavior of the memory function arises from several physical features of intermediate scale correlations and dynamics: liquid homogeniety $S(k) \cong S(0)$, self-similiar correlations $\omega(k) \sim(k \sigma)^{-2}$, and Brownian decay of fluctuating forces as $\ln \left\{F^{\mathrm{R}}(k, t)\right\} \sim-k^{2} t^{1 / 2}$, or $\ln \left\{F^{\mathrm{R}}(k, t)\right\} \sim-k^{4} t$ based on the Akcasu form of Eq. (2.12).

There are many consequences of the time-dependent excluded volume force friction $^{36)}$. In the long time Markovian regime, the zero frequency part of the RR friction constant is proportional to $\psi\left(N^{2} / R_{\mathrm{g}}^{3}\right) \sim \psi N^{1 / 2}$. The $N$-dependent enhancement factor is of geometric origin, being proportional to the number of contacts between a pair of random coil chains in 3-dimensions, which are dynamically correlated until the chains have diffused a distance of order the radius-of-gyration or correlation hole length scale. Mathematically, this factor arises from $k \cong R_{\mathrm{g}}^{-1}$ scale contributions to the time integral of $\Delta \zeta(t)$ associated with the $|\alpha-\gamma| \gg 1$ contributions of segments widely separated in a chemical interval sense. The corresponding RR predictions for the longest chain relaxation time, viscosity, and diffusion constant are all modified by this factor

$$
\frac{\tau_{\mathrm{RR}}}{\tau_{\mathrm{R}}}=\frac{\eta_{\mathrm{RR}}}{\eta_{\mathrm{R}}}=\frac{D_{\mathrm{R}}}{D_{\mathrm{RR}}}=1+a \psi \sqrt{N}, \quad \psi \equiv \rho \sigma^{3} g^{2}(\sigma) S_{0}
$$


where " $a$ " is a property-specific numerical constant of order unity and $S_{0} \equiv S(k=0)$. Relaxation and diffusion on intermediate time scales, $\tau_{\mathrm{c}} \ll t \ll \tau_{\mathrm{R}}$, are also slowed down. For example, the chain-averaged segmental and center-of-mass displacements, $\mathrm{g}(t)=\left\langle(\boldsymbol{r}-\boldsymbol{r}(t))^{2}\right\rangle$ and $g_{\mathrm{CM}}(t)=\left\langle\left(\boldsymbol{R}_{\mathrm{CM}}-\boldsymbol{R}_{\mathrm{CM}}(t)\right)^{2}\right\rangle$, respectively, can be determined from the Laplace space relations

$$
\begin{aligned}
& \begin{aligned}
g(z) & =\int \mathrm{d} p\left\{z^{-1} C_{\mathrm{p}}(0)-C_{\mathrm{p}}(z)\right\} \\
& =\frac{\sigma^{2}}{6 N} \int \mathrm{d} p \frac{1}{z\left\{z K_{\mathrm{S}}^{-1}\left(\zeta_{0}+\Gamma_{p}(z)\right)+(p \pi / N)^{2}\right\}}
\end{aligned} \\
& g_{\mathrm{CM}}(z)=N^{-1} \frac{\sigma^{2}}{z^{2} \beta\left[\zeta_{0}+\Gamma_{0}(z)\right]}
\end{aligned}
$$

from which one obtains: $\mathrm{g}(t) \sim t^{3 / 8}$ and $g_{\mathrm{CM}} \sim \mathrm{t}^{3 / 4}$. For the $t>\tau_{\mathrm{R}}$ Markov regime, Rouse-like behavior is recovered but with an effective segment friction constant enhanced by a factor proportional to $\psi N^{1 / 2}$. Thus, $g(t) \sim(t / \sqrt{N})^{1 / 2}$ for $\tau_{\mathrm{R}} \ll t \ll \tau_{\mathrm{RR}}$, and $g(t) \sim D_{\mathrm{RR}} t$ for $t \gg \tau_{\mathrm{RR}}$. The time dependence of other dynamic properties are also modified in the intermediate regime, and the $C_{\mathrm{p}}(t)$ functions decay in a nonexponential manner ${ }^{36)}$. Qualitatively, the slowing down of $g(t)$ and $g_{\mathrm{CM}}(t)$ at intermediate times can be thought of as arising from a time-dependent friction coefficient, $\zeta(t)$ $=\zeta_{0}+\int_{0}^{t} \mathrm{~d} t^{\prime} \Delta \zeta\left(t^{\prime}\right)$, although such a "pseudo-Markov" perspective is not rigorous.

For large enough $N$, RR theory predicts strong deviations from Rouse dynamics. Many of these predictions are in reasonable accord with some Monte Carlo simulations of lightly entangled chains ${ }^{14,36}$. The RR model has also been successfully employed by Fatkullin and Kimmich to interpret short and intermediate time dynamics of entangled melts as probed by field cycling NMR experiments ${ }^{64)}$. However, RR theory does not agree with most experiments on strongly entangled systems. This is not unexpected due to the dynamically perturbative nature of this theory. Nevertheless, the RR model has found use in two different contexts. First, as a technical approximation for the projected dynamics entering a mode-coupling theory of the memory functions ${ }^{36,37)}$ as discussed in the section IID. Secondly, as a tool to determine conditions for the failure of Rouse theory, which is then employed as a criterion for the emergence of entanglement dynamics ${ }^{39,42)}$. Such a criterion can be implemented in the time domain to estimate the unentangled-entangled crossover time scale (length scale), $\tau_{\mathrm{c}}\left(r_{\mathrm{c}}\right)$, or implemented at the Markovian effective friction level to estimate the critical entanglement degree of polymerization $N_{\mathrm{e}}$. Equating the corresponding Rouse and RR contributions to the friction yields the semiquantitative

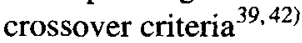

$$
\int_{0}^{\tau_{\mathrm{c}}} \mathrm{d} t \Delta \zeta(t) \cong \zeta_{0} \quad \text { or } \quad \psi \sqrt{N_{\mathrm{e}}} \cong 1
$$


Using the RR results discussed above yields

$$
\begin{gathered}
\tau_{\mathrm{c}} \sim \sigma^{2} \beta \zeta_{0} \psi^{-4} \Rightarrow r_{\mathrm{c}}^{2} \sim \sqrt{\frac{\tau_{\mathrm{c}}}{\beta \zeta_{0}}} \sim \sigma^{2} \psi^{-2} \sim \sigma^{2} N_{\mathrm{e}} \\
G_{\mathrm{N}} \equiv \frac{\rho k_{\mathrm{B}} T}{N_{\mathrm{c}}} \sim \rho k_{\mathrm{B}} T \psi^{2}
\end{gathered}
$$

where the crossover length scale ("tube diameter" in reptation theory) and plateau shear modulus, $G_{\mathrm{N}}$, are estimated by evaluating Rouse theory at the predicted crossover time. The key physical quantity is the dimensionless force strength parameter, $\psi=\rho \sigma^{3} g^{2}(d) S(0)$, which is determined by local interchain packing and collective density fluctuations $S(0)=S_{0}=\rho \beta^{-1} \kappa$, where $\kappa$ is the isothermal compressibility.

The required structural quantities can be theoretically evaluated at various levels of chemical realism using PRISM theory ${ }^{42,57)}$. For example, based on the simplest Gaussian thread or string model of polymer chains, analytic PRISM theory predicts $\xi_{\rho} \sim\left(\rho \sigma^{2}\right)^{-1}, S_{0} \sim\left(\xi_{\rho} / \sigma\right)^{2}, g(\sigma) \sim \rho \sigma^{3} \sim \psi$ for semidilute, and perhaps concentrated, polymer solutions ${ }^{42)}$. What is remarkable is the power law density dependence predicted by the microscopic PRISM theory, which agrees with blob scaling results $^{2)}$ for the density screening length and $S_{0}$. Using these results in Eqs. (2.17) yields $^{42)}$

$$
\tau_{c} \sim \tau_{0}\left(\rho \sigma^{3}\right)^{-4}, \quad r_{\mathrm{c}} \sim \xi_{p}, \quad N_{\mathrm{e}} \sim\left(\rho \sigma^{3}\right)^{-2}, \quad G_{\mathrm{N}} \sim\left(\rho \sigma^{2}\right)^{3}
$$

which for good solvents (where $\sigma \sim \rho^{-1 / 8}$ ) implies

$$
G_{\mathrm{N}} \sim \rho^{9 / 4}, \quad N_{\mathrm{e}} \sim \rho^{-5 / 4}, \quad r_{\mathrm{c}} \sim \rho^{-3 / 4}
$$

in agreement with dynamical scaling approaches ${ }^{2-4)}$. Curiously, the prediction that the plateau shear modulus is proportional to the cube of the chemical-structuredependent inverse "packing length", $\rho \sigma^{2} \sim R_{\mathrm{g}}^{2} / V$ where $V$ is the spacing filling volume of a single polymer, is in complete agreement with the recent comprehensive study of entangled melts ${ }^{65}$. Predictions for theta solvents, possible complications under concentrated solution conditions, and additional comparisons with experiments and other theoretical approaches have also been discussed ${ }^{42}$.

\section{Macromolecular mode-mode-coupling theory}

The description of the slow entanglement dynamics in real systems requires going beyond the perturbative RR approach. The complex many body physics is contained in the memory function matrix, $\Gamma_{a \gamma}(t)$, and must somehow be extracted. Mode-coupling theory ${ }^{50-53)}$ is an approximate attempt to do this based on identifying the relevant "slow variables". For hydrodynamic problems, conserved collective variables (mass, momentum, energy) are the obvious choices. However, for other problems such as local caging in atomic and colloidal liquids, dynamic percolation, and the glass transition, the relevant variables are not so obvious. For the polymer problem, 
the key physical point is that entanglement caging is a process which is expected to induce highly cooperative motions of all $N$ segments on a tagged polymer, in strong contrast to simple Rouse theory. Thus, to describe conformational and (single chain) stress relaxation, and all internal chain modes, it was argued that it is essential to keep track of every tagged polymer segment and how it is influenced ("caged") by excluded volume interactions with the surrounding polymer matrix ${ }^{36)}$. This motivates retaining all $N$ tagged polymer positions as slow variables. In a Fourier field variable representation this choice corresponds to

$$
\left\{\rho_{k}^{\alpha}(t)\right\}, \quad \rho_{k}(t)=\int_{0}^{N} \mathrm{~d} \alpha \rho_{k}^{\alpha}(t)=\int_{0}^{N} \mathrm{~d} a \exp \left(\mathrm{i} k \cdot r_{a}(t)\right)
$$

The collective single polymer density, $\rho_{k}$, is also defined above. If one is interested in describing only center-of-mass motion, then retaining the scalar collective single chain density is adequate, and leads to predictions for the translational diffusion constant which are virtually identical to those obtained by a full analysis retaining all internal modes ${ }^{45,47)}$.

For the matrix polymers, there are (at least) two possibilities for the slow collective variable: (i) total density fluctuations ${ }^{36}$, or (ii) collective microscopic stress variable $^{48)}$. In the Fourier field representation these are

$$
\begin{aligned}
& c_{k}(t)=\sum_{j=1}^{n_{\mathrm{M}}} \int_{0}^{N} \mathrm{~d} a \exp \left(\mathrm{i} \boldsymbol{k} \cdot \boldsymbol{r}_{a}(t)\right) \\
& \sigma_{k}(t)=-\sum_{j=1}^{n_{\mathrm{M}}} \int_{0}^{N} \mathrm{~d} a k^{-1}\left(\beta^{-1} \boldsymbol{k}-\mathrm{i} \boldsymbol{F}_{a}^{j}(t)\right) \exp \left(\mathrm{i} \boldsymbol{k} \cdot \boldsymbol{r}_{a}^{j}(t)\right)
\end{aligned}
$$

where $n_{\mathrm{M}}$ is the number of matrix polymers, and all forces (intra- and intermolecular) enter the vectorial stress variable the autocorrelation of which is the collective wavevector-dependent stress tensor. In the original formulation of Schweizer ${ }^{36}$, and most of the subsequent PMC work, choice (i) was employed. However, more recent analysis by Fuchs and Schweizer ${ }^{48)}$ has employed choice (ii). There is virtually no qualitative difference between the two choices, especially for purposes of deriving qualitative predictions in the large $N$ asymptotic limit. However, for analysis of the essential finite $N$ corrections ${ }^{48}$, the collective stress variable is preferable since it leads most naturally to memory function expressions with parameters directly related to experiment.

To extract the slow, cooperative tagged polymer dynamics induced by interchain excluded volume forces, $N$ bilinear variables are constructed which are products of the slow tagged chain segment and collective fields.

$$
\left\{\rho_{k}^{\alpha} c_{-k}\right\} \quad \text { or } \quad\left\{\rho_{k}^{a} \sigma_{-k}^{\mathrm{a}}\right\} \quad \alpha=1,2 \ldots N
$$

where the superscript "a" on the stress variable refers to its longitudinal component (parallel to wavevector $k$ ). If only the center-of-mass motion of the tagged chain is of interest, then an adequate, and much simpler, alternative is the single bilinear variable 


$$
\left\{\rho_{k} c_{-k}\right\} \quad \text { or } \quad\left\{\rho_{k} \sigma_{-k}^{\mathrm{a}}\right\}
$$

Interchain forces are expressable in terms of these bilinear variables. The mode-coupling methodology projects the excluded volume forces in the memory function matrix $\Gamma_{a \gamma}(t)$ onto these slow product variable(s) in order to approximately extract the collective dynamical contributions. Four-point correlations are factorized into products of 2-point correlations. For the general problem, the presence of $N$ slow variables introduces novel technical complications which are analytically treatable based on matrix diagonalization and inversion techniques for Gaussian fractal type macromolecular models ${ }^{37,47)}$. For example, the inverse of $\omega_{a \gamma}(k)$ is a tridiagonal matrix for Gaussian linear chains ${ }^{61)}$. Mathematical tractability and physical arguments motivate the following approximation at the matrix level ${ }^{36}$ )

$$
\omega^{-1}(k) \omega^{Q}(k, t) \omega^{-1}(k) \cong \omega^{-1}(k) F^{Q}(k, t)
$$

where $F^{Q}(k, t)$ is the coherent single chain propagator. This simplification corresponds to the physical assumption that only coherent single chain motions are effective in relaxing the constraining entanglement forces, and the same dynamical processes relax the forces which impede center-of-mass diffusion and conformational relaxation.

Ignoring chain end effects yields the probe chain continuum GLE's ${ }^{37)}$

$$
\begin{aligned}
\zeta_{0} \frac{\partial \boldsymbol{r}_{\alpha}(t)}{\partial t} & +\int_{0}^{t} \mathrm{~d} \tau \Sigma(t-\tau) \frac{\partial \boldsymbol{r}_{\alpha}(\tau)}{\partial \tau}+\int_{0}^{t} \mathrm{~d} \tau M(t-\tau) \frac{\partial}{\partial \tau} \frac{\partial^{2} \boldsymbol{r}_{\alpha}(\tau)}{\partial \alpha^{2}} \\
& =K_{\mathrm{S}} \frac{\partial^{2} \boldsymbol{r}_{\alpha}(t)}{\partial \alpha^{2}}+f_{a}(t)+\boldsymbol{F}_{\alpha}^{Q}(t)
\end{aligned}
$$

Two new caging forces are predicted to modify the Rouse equations-of-motion in an elastic or viscous manner depending on time scale. The first is quantified by a "diffusional" memory function $\Sigma(t)$, and describes a time-delayed, uniform drag on each segment. Such a delayed caging force would also arise for simple atomic fluids or colloidal suspensions of spherical particles. This contribution is of the same general form as in RR theory, and will be the primary origin of anomalous segmental and center-of-mass diffusion. The center-of-mass translational diffusion constant is entirely determined by the zero frequency part of $\Sigma(t)$. The truly novel caging force is quantified by the "viscoelastic" memory function $M(t)$. It describes a timedelayed force sensitive to the rate of change of the elastic spring forces with time. This force strongly modifies conformational and stress relaxation, and ascribes a history-dependent penalty for changing polymer shape or orientation. Thus, it produces conformational caging or temporary localization. Curiously, in a Markovian limit it is of the empirical Cerf "internal friction" form ${ }^{2,66)}$, and also bears a striking resemblence to various empirical approaches which have successfully been used to fit entangled polymer melt data ${ }^{66)}$.

Although the general form of the GLE's do not depend on the different possible choices of slow bilinear variables, the memory function expressions are weakly 
dependent. The original PMC theory employed the collective matrix density and $N$ tagged polymer segment densities as slow variables with the result ${ }^{36,37)}$

$$
\begin{aligned}
& \Sigma(t) \cong\left(72 \pi^{2} \beta \sigma^{-2}\right)^{-1} \int_{0}^{\infty} \mathrm{d} k k^{6} \omega^{2}(k) C^{2}(k) \rho S(k) F^{Q}(k, t) F_{\text {Coll }}^{Q}(k, t) \\
& M(t) \cong\left(2 \pi^{2} \beta \sigma^{2}\right)^{-1} \int_{0}^{\infty} \mathrm{d} k k^{2} \omega^{2}(k) C^{2}(k) \rho S(k) F^{Q}(k, t) F_{\text {Coll }}^{Q}(k, t)
\end{aligned}
$$

All the terms in the wavevector integrals were defined when the RR theory was discussed except for $C(k)$, which is the repulsive force site-site intermolecular direct correlation function defined by the PRISM integral equation ${ }^{57)}$ as: $h(k)=$ $\omega(k) C(k) S(k)$. The $t=0$ values of the integrands quantify the amplitude or strength of dynamical constraints on a length scale $2 \pi / k$. They provide precise connections to the equilibrium structure, which quantify the influence of spatial correlations of the matrix forces on the polymer motion, and are characterized by both the macromolecular length scale, $R_{\mathrm{g}}$, and local nonuniversal length scales. The much stronger weighting (see Fig. 3) of macromolecular scale correlations in $M(t)$ compared to $\Sigma(t)$ leads to very different consequences of entanglements on translational diffusion versus conformational and stress relaxation.

Temporal decay of the memory functions corresponds to the entanglement relaxation process, and proceeds via three parallel, length-scale-dependent mechanisms: (i) tagged polymer center-of-mass motion, (ii) tagged polymer internal mode dynamics or shape fluctuations, and (iii) collective matrix relaxation. A naive "frozen matrix" approximation ${ }^{36)}$ corresponds to $F_{\text {coll }}^{Q}(k, t) \cong 1$. These three processes might be crudely interpreted as the PMC analogs of probe reptation (a coherent pro-

Fig. 3. Reduced wavevector dependence of the $t=0$ entanglement constraint amplitudes in the large $N$ asymptotic limit for the diffusional $\Sigma$ (solid line, scaled by $1 / 10$ ) and viscoelastic $M$ (long dashes) memory functions of Eqs. (2.28) and (2.29), respectively. The corresponding asymptotic friction amplitudes associated with the zero-frequency limit of the memory functions of the diffusional (chain curve) and viscoelastic (shortdashed) memories are also shown as the $\Gamma$ curves

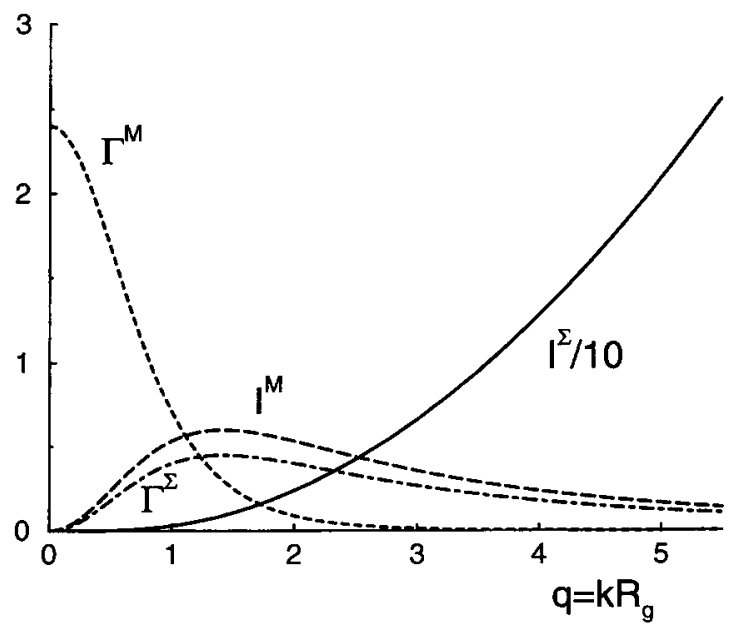


cess), contour-like fluctuations, and constraint release within the tube model approach. Note that the most important decay channel generally depends on $N$ and length scale $2 \pi / k$. Moreover, transport properties cannot generally be expressed as a sum of independent values associated with different decay mechanisms of the entanglement forces. In contrast with phenomenological approaches ${ }^{2-4)}$, multiple transport mechanisms at the chain trajectory level are not postulated. Rather, the focus is on the statistical correlations of the entanglement forces which are influenced by multiple structural length scales and time-dependent decay channels.

Recent analysis of Fuchs and Schweizer based on the collective stress as the matrix slow variable, plus an explicit treatment of the center-of-mass motion, yields $^{48)}$

$$
\begin{aligned}
& \Sigma(t) \cong\left(6 \pi^{2} \beta\right)^{-1} \int_{0}^{\infty} \mathrm{d} k k^{4} \omega(k) C^{2}(k) \rho S(k)\left[\frac{\rho S(k)}{\beta G(k)}\right] F^{Q}(k, t) F_{G}^{Q}(k, t) \\
& M(t) \cong\left(2 \pi^{2} \beta \sigma^{2}\right)^{-1} \int_{0}^{\infty} \mathrm{d} k k^{2} \omega^{2}(k) C^{2}(k) \rho S(k)\left[\frac{\rho S(k)}{\beta G(k)}\right] F^{Q}(k, t) F_{\mathrm{Coll}}^{Q}(k, t)
\end{aligned}
$$

where the normalized propagator for collective stress relaxation is indicated by the subscript " $G$ ". Qualitatively for all $N$, and rigorously in the large $N$ Markovian limit, these memory functions are very similiar to Eqs. (2.26) and (2.27). In the intermediate wavevector regime, the factor $k^{2} \omega(k) / 12 \rightarrow 1$ in Eq. (2.26) and $[\rho S(k) /(\beta G(k))] \cong$ $S_{0}$, thereby implying the wavevector dependence of the two sets of integrands of $\Sigma(t)$ and $M(t)$ in Eqs. (2.26)-(2.29) are identical. As discussed in section IID below, the time dependences are also virtually identical, in either the frozen matrix limit or in the presence of constraint release. Differences are primarily at the nonuniversal prefactor level, and at extremely long times where the long time tail of $\Sigma(t)$ differs in Eqs. (2.26) and (2.28). However, this last aspect has no real consequences for observable properties ${ }^{47,48}$.

As emphasized in Fig. 3, for large $N$ the viscoelastic memory function $M(t)$ is dominated by contributions on the macromolecular scale, $k R_{\mathrm{g}} \cong 1$. Its amplitude on this scale is proportional to the number of pair contacts between two interpenetrating polymers, a geometric factor proportional to $N^{2} / R_{\mathrm{g}}^{3} \sim N^{1 / 2}$. Such $k R_{\mathrm{g}} \cong 1$ contributions decay very slowly in time. Thus, in the GLE's of Eq. (2.25), for $N \gg 1, M(t)$ is very large and nearly constant. Based on the crossover analysis of section IIB, $M(t=0) \sim \rho S(0) C^{2}(0) N^{1 / 2} \sim\left(\rho S_{0}\right)^{-1} N^{1 / 2} \sim\left(N / N_{\mathrm{e}}\right)^{1 / 2}$. The corresponding term in the GLE acts an an elastic restoring, or conformational caging, force. Integrating by parts yields

$$
M(t=0)\left[\frac{\partial^{2} r_{\alpha}(t)}{\partial a^{2}}-\frac{\partial^{2} r_{\alpha}(0)}{\partial a^{2}}\right] \underset{M(t=0) \sim \sqrt{N / N_{e}} \rightarrow \infty}{\longrightarrow} \frac{\partial^{2} r_{\alpha}(t)}{\partial a^{2}}=c_{\alpha}
$$

where $c_{\alpha}$ is the initial value of the elastic restoring force exerted on segment $\alpha$. The idea is that in the large $N$ limit the "spring constant" for maintaining a constant chain curvature diverges, and hence the corresponding term in the GLE's can be treated as a constraint. Hence, this caging force leads to the prediction of conformational and 
stress relaxation arrest, the hallmark of entanglement. In the asymptotic large $N$ limit, Eq. (2.30) can be qualitatively interpreted as providing a constraint on the tagged chain motions which induces highly cooperative $N$ body motions. Isotropic, random displacements of two segments can occur, but the remaining $N-2$ segments must move in a cooperative, deterministic manner in order to satisfy the vectorial constraints of constant elastic force or backbone curvature: $\boldsymbol{r}_{a+1}+\boldsymbol{r}_{a-1}-2 \boldsymbol{r}_{a}=\boldsymbol{c}_{a}=$ constant for all $\alpha$.

Fig. 4 shows a highly schematic example of the cooperative motions induced by the viscoelastic caging force in the large $N$ limit ${ }^{48)}$. Two segments are displaced by an arbitrary amount and direction, and the remaining $N-2$ segments move cooperatively to maintain constant local curvature. Visualization at the chain trajectory level is more difficult than for the reptation mechanism, and Fig. 4 represents only one of many possible collective motions for a small time step. Despite the near conformational (chain curvature) localization, segments can still diffuse, but in an anomalous, highly constrained fashion determined by the time fractal form of the $\Sigma$ memory function. This scenario is not unlike reptation where the end segments can randomly reorient, but the other segments coherently reptate along their contour and diffusion relative to the laboratory fixed axes still occurs but in a much slowed down fashion. For a given set of initial conditions, PMC theory does predict anisotropic elementary motions in order to satisfy the vectorial constraints in Eq. (2.30). However, displacements of two segments are allowed to randomly occur, thereby restoring isotropy in an average sense. Also note that the local curvature conserving motions do not correspond to a rigid body motion or rotation which would require the stronger constraints that $\left|\boldsymbol{r}_{\alpha}(t)-\boldsymbol{r}_{\gamma}(t)\right|=$ constant for all $a y$ pairs and all times. PMC theory predicts (not assumes) that bonds can stretch and bend, and the coil shape can fluctuate, if (nearly) constant local stress and chain backbone curvature is maintained.

Fig. 4. Schematic example of consequences on a discrete bead-spring polymer trajectory of the viscoelastic constraint of constant chain curvature (Eq. (2.30) with $c_{a}=$ 0 ) for pre-terminal relaxation times. All beads move in a coherent, anisotropic manner once the displacements, $\boldsymbol{u}$ and $v$, of two randomly selected beads are arbitrarily chosen. The size of displacements and segments are not drawn to absolute scale; only the relative magnitude and direction of segmental displacements are relevant

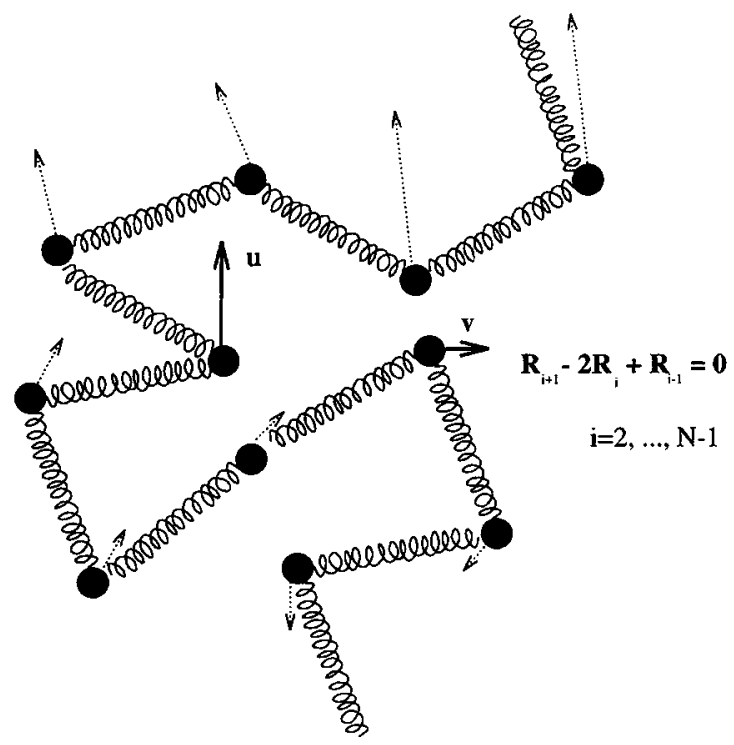


Many observable properties are expressable in terms of the normal mode time correlation functions, $C_{p}(t)$, which follow from diagonalization of Eqs. (2.25) by the Rouse modes and Laplace transformation as ${ }^{37)}$

$$
\frac{C_{p}(z)}{C_{p}(t=0)}=\left\{z+K_{\mathrm{S}} \frac{(p \pi / N)^{2}}{\zeta_{0}+\Sigma(z)+(p \pi / N)^{2} M(z)}\right\}^{-1} \underset{p \gg 1}{\longrightarrow}\left(z+\frac{K_{\mathrm{S}}}{M(z)}\right)^{-1}
$$

The corresponding $p$-mode relaxation times are given by

$$
\tau_{p} \equiv \int_{0}^{\infty} \mathrm{d} t \frac{C_{p}(t)}{C_{p}(0)}=M(z=0)+\left(\frac{N}{p \pi}\right)^{2}\left(\zeta_{0}+\Sigma(z=0)\right)
$$

Note that for most internal chain modes, the normalized time correlation function is independent of normal mode index and hence characteristic length scale. This interesting property is consistent with the interpretation in terms of a localized conformational state, and the physical requirement that a finite fraction of the chain internal modes must be simultaneously arrested by entanglement constraints in order that an $N$-independent plateau shear modulus emerges.

An important caveat is that any mode-coupling theory for any physical problem is not valid at arbitrary short times and distances since it is only designed to describe collective, slow processes ${ }^{50-53)}$. As a simple time domain crossover model, we assume that Rouse dynamics holds up to the time $\tau_{c}$ computed using RR theory in section B. Beyond this time, Rouse theory is predicted to fail and PMC theory is invoked. A more quantitative numerical approach to this crossover problem is discussed in section VI.

\section{Entanglement force memory functions}

For precise predictions the projected dynamic propagators describing entanglement force relaxation are required. This is a very difficult problem in nonequilibrium statistical mechanics which is attacked based on various, usually uncontrolled, approximations ${ }^{50-53)}$. The classic approaches correspond to dynamic perturbation theory which for our problem would imply Rouse dynamics, or a self-consistent approach where the projected dynamics is replaced by the full physical dynamics. The former approach underestimates friction effects, while outside the hydrodynamic regime the latter overestimates the consequences of constraining forces. For example, self-consistent mode coupling theory of Götze et al. predicts an “ideal glass transition" upon increasing particle density and stronger local caging ${ }^{52)}$. A (power law) divergence of the structural relaxation time associated with the spatially-resolved collective density fluctuation correlator is predicted. Whether such a strong feedback mechanism exists for the entanglement problem at the single chain level is certainly not obvious, particularly for the linear Rouse mode time correlation functions. Moreover, for the glass problem one is interested in increases of the viscosity by $13-14$ orders of magnitude. In contrast, for the entangled polymer pro- 
blem the corresponding enhancement (suppression) of the shear viscosity (diffusion constant) relative to unentangled Rouse behavior is typically only 4 or 5 (2) orders of magnitude for experimentally accessible degrees of polymerization. Within PMC theory, the self-consistent approach has been implemented by Schweizer at the technically crudest level ${ }^{39}$ ). A dynamic localization transition on the macromolecular scale is predicted at very high $N$, the physical significance of which remains unclear.

Lacking in the general case an a priori argument, the only real justification for any specific approximation is the veracity of the results it produces. The approach adopted in the original PMC analysis ${ }^{36,37)}$, and subsequently shown to produce many predictions in accord with experiments ${ }^{36-48)}$, is an intermediate type approximation. The projected single chain dynamics is approximated by the RR theory which accounts for entanglement effects (at the memory function level) in a short time manner; the projected collective propagator is assumed to follow the real dynamics in a self-consistent spirit ${ }^{36,37)}$. It is perhaps worth emphasizing that PMC theory employs one set of approximations for the fundamental segmental forces which is used to predict the dynamical behavior of all observables over a very wide range of length and frequency scales.

It is interesting to contrast the above approach with very recent theoretical work by Verberg, deSchepper and Cohen ${ }^{67)}$ concerning the viscosity of (hard core) colloidal suspensions. Based on kinetic theory and mode-coupling arguments, the relaxation time of the excluded volume fluctuating forces for this problem was approximated in a short time, Enskog type manner (independent binary collisions) corrected only by the strong local static correlations associated with $S(k)$ at high densities. When this approximation for the dynamics was employed in a mode-coupling expression for the shear viscosity, excellent agreement with experiment was found over a wide range of packing fractions from dilute suspensions up to dense liquids. Over this range of density, local collective caging processes result in a factor of nearly 100 increase of the suspension viscosity. It would seem that the RR approach for the polymer problem is an approximation in the same spirit as this non-self-consistent statistical dynamical approach to hard sphere fluids ${ }^{67)}$.

Since RR dynamics is simpler than real entangled dynamics, one can immediately draw two conclusions based on its use as an approximation for the projected propagators. (i) In the $N \rightarrow \infty$ long chain limit, memory function relaxation is controlled entirely by RR probe motions and matrix "constraint release" effects are negligible. This feature is in qualitative accord with the reptation/tube ideas. (ii) The fluctuating force memory functions obey "simpler" dynamics (the basis of traditional Brownian motion theory ${ }^{50)}$ ), and relax faster than the true single chain entangled dynamics. However, at non-infinite $N$ there may be strong corrections, or even a breakdown (non-Markovian situation), to this "separation of time scales" behavior. Technically, points (i) and (ii) follow for $N \gg 1$ since $\tau_{\mathrm{RR}} \sim N^{5 / 2}$ but (as discussed in section IIIA) the collective density fluctuation and shear stress relaxation times scale as $\tau \sim N^{3}$.

The above ideas are mathematically expressed for the coherent single chain propagator by using the simple form ${ }^{42,63)}$ 


$$
F^{\mathrm{RR}}(k, z) \cong\left\{z+\frac{k^{2}}{\beta \omega(k)\left[\zeta_{0}+\Delta \zeta(z)\right]}\right\}^{-1}
$$

where $\Delta \zeta(t)$ is given by the RR expression in Eqs. (2.11)-(2.13). The PMC memory functions then display three distinct time regimes: $\tau_{\mathrm{c}} \ll t \ll \tau_{\mathrm{R}}, \tau_{\mathrm{R}} \ll t \ll \tau_{\mathrm{RR}}$, and $t$ $\gg \tau_{\mathrm{RR}}$, associated with the RR dynamics.

The collective density fluctuations are modeled as ${ }^{48)}$

$$
S(k) \cong \frac{S_{0}}{1+k^{2} \xi_{\rho}^{2}}, \quad F_{\mathrm{coll}}(k, t) \equiv \frac{S(k, t)}{S(k)} \underset{t \gg \tau_{\mathrm{c}}}{\longrightarrow} f_{k}^{S} \Phi(t)
$$

where $\xi_{\rho}$ is the polymer density screening length or mesh size, and the dynamic propagator decays rapidly (via cooperative diffusion) to a temporary wavevector dependent plateau which is significantly less than unity (but $N$-independent). The latter relaxes only at very long times corresponding to complete chain disentanglement. The original PMC analysis effectively assumed $f_{k}^{S}=1$, a naive frozen matrix approximation $^{36)}$. The collective shear stress relaxation function is modeled as ${ }^{48}$ )

$$
G(k) \cong \rho \beta^{-1} \frac{S(k)}{S_{0}}, \quad F_{G}(k, t) \underset{i \gg \tau_{\mathrm{c}}}{\longrightarrow} f_{k}^{G} \Phi(t)
$$

Following the analysis of many workers ${ }^{15,68,69)}$, the amplitude of the transient plateau, and the terminal decay function, are taken as

$f_{k}^{S} \cong \frac{S_{0}}{N_{\mathrm{e}}} \exp \left(-k^{2} r_{\mathrm{c}}^{2} / 36\right), \quad f_{k}^{G} \cong N_{\mathrm{e}}^{-1} \exp \left(-k^{2} r_{\mathrm{c}}^{2} / 36\right), \quad \Phi(t)=\exp \left(-t / \tau_{\mathrm{D}}\right)$

where $S_{0} / N_{\mathrm{e}}$ is the nonuniversal ratio of the shear to bulk modulus and $\tau_{\mathrm{D}}$ is the terminal relaxation time. This ratio is independent of polymer concentration in semidilute good solvents ${ }^{70)}$, and decreases strongly in concentrated and melt states due to the suppression of polymer density fluctuations. The wavevector dependence of the amplitude factors is of Gaussian form in qualitative accord with prior analyses ${ }^{69)}$. The dynamic crossover length $r_{c}$ is a nonuniversal, density-dependent intrinsic length scale beyond which elastic transient network like behavior occurs in entangled fluids. A simple, $\mathrm{k}$-independent form ${ }^{3,37,68,69)}$ for the terminal relaxation function is adopted which is characterized by a single shear stress relaxation time $\tau_{D}$.

Consider first the behavior of the PMC memory functions in the asymptotic large $N$ regime, where two major simplifications occur. (1) Collective constraint release processes are not important, and RR single chain dynamics controls the time-dependence of the memory functions. (2) Local length scales drop out in the sense that $r_{\mathrm{c}} / R_{\mathrm{g}}, \xi_{\rho} / R_{\mathrm{g}}$, and $\sigma / R_{\mathrm{g}} \rightarrow 0$, and are thus irrelevant in the determination of the amplitude of constraints in the memory functions. Straightforward analysis ${ }^{37,41)}$ then yields for the time dependence of the diffusional memory function of Eq. (2.28): 


$$
\begin{aligned}
\Sigma(t) & \sim t^{-9 / 16} & & \text { for } \quad \tau_{\mathrm{c}} \ll t \ll \tau_{\mathrm{R}} \\
& \sim(\sqrt{N} / t)^{3 / 4} & & \text { for } \quad \tau_{\mathrm{R}} \ll t \ll \tau_{\mathrm{RR}} \\
& \sim N\left(N^{3 / 2} / t\right)^{5 / 2} & & \text { for } \quad t \gg \tau_{\mathrm{RR}}
\end{aligned}
$$

and for the viscoelastic memory function of Eq. (2.29)

$$
\begin{aligned}
M(t) & \sim \sqrt{N}-a t^{3 / 16} & & \text { for } \quad \tau_{\mathrm{c}} \ll t \ll \tau_{\mathrm{R}} \\
& \sim \sqrt{N}-b(t / \sqrt{N})^{1 / 4} & & \text { for } \quad \tau_{\mathrm{R}} \ll t \ll \tau_{\mathrm{RR}} \\
& \sim N^{2}\left(N^{3 / 2} / t\right)^{3 / 2} & & \text { for } t \gg \tau_{\mathrm{RR}}
\end{aligned}
$$

where $a$ and $b$ are $N$-independent, system-specific prefactors.

The diffusional memory function displays fractional power law decay over intermediate time intervals due to the self-similiar nature of the intra- and intermolecular correlations, the homogeneity of the liquid on scales larger than $r_{c}$, and the diffusive RR segmental dynamics (shape fluctuations) which determines force relaxation. In essence, no well-defined elementary diffusive "jump time" exists. Anomalous segmental and center-of-mass diffusion is thus predicted.

The time dependence of the viscoelastic memory function is particularly unusual. Out to the longest fluctuating force relaxation time, $\tau_{\mathrm{RR}}$, the amplitude is nearly frozen at a value which grows indefinitely with $N$ and is proportional to the number of correlated contacts between a pair of interpenetrating random coils in 3-dimensions. It is this leading contribution which generates conformational localization and a shear stress plateau. Dynamic polymer shape fluctuations generate a weak, finite size decay of $M(t)$, which remarkably is of the so-called von Schweidler form familiar from glassy dynamics ${ }^{52}$. Physically, such a weak decay describes the early and intermediate stages of entanglement force relaxation via sub- $R_{\mathrm{g}}$ scale motions (shape fluctuations) of the tagged polymer. The final long time tail arises from the presumed diffusive decay of the force correlations for $k R_{\mathrm{g}}<1$ due to probe center-ofmass translation.

Finally, we point out that although the very long time tails of the memory functions are "integrable" and produce negligible contributions in the Markovian limit of zero frequency, they can have non-negligible consequences for the low frequency domain response functions. However, they are "cuttoff" by constraint release processes $(\Phi(t)$ in Eq. (2.36)) which on the relevant length scales are wavevector-independent. That is, the memory functions have the form $t^{-x} \exp \left(-t / \tau_{D}\right)$ for long times $t$ $\gg \tau_{\mathrm{RR}}$.

\section{Asymptotic predictions for chain solutions and melts}

The asymptotic predictions of PMC theory follow directly from the memory functions, GLE's, and the entanglement onset model described in section II. The most basic results are summarized in this section. 


\section{A. Transport coefficients and terminal relaxation times}

Transport coefficients and relaxation times are determined by the zero frequency (Markovian) limit of the memory functions. In analogy with dynamic critical phenomena ${ }^{53,54)}$, their asymptotic large $N$-dependences are deducable based on simple power counting arguments, i.e. nondimensionalization of the wavevector integrals in the memory functions by the macromolecular size $R_{\mathrm{g}}$. The results are: $\Sigma(z=0)$ $\sim N$ and $M(z=0) \sim N^{3}$. Although $R_{\mathrm{g}}$ scale contributions control the qualitative form of the Markovian limit of both memory functions, recall that the viscoelastic caging forces are much less sensitive to sub- $R_{\mathrm{g}}$ correlations than the diffusional memory $\Sigma(t)$. The relative importance to the Markovian friction of contributions on the scale $k$ are also shown in Fig. 3.

The predictions for self-diffusion, terminal relaxation time, shear viscosity, and compliance, $J_{\mathrm{e}}$, based on the original collective density slow variable choice and the naive frozen matrix approximation are ${ }^{37,39-43)}$ :

$$
\begin{aligned}
& D \sim\left(\beta \zeta_{0} g^{2}(\sigma) N^{2}\right)^{-1}, \quad \tau_{D} \sim \tau_{0} g^{2}(\sigma) N^{3}, \\
& \eta \cong G_{N} \tau_{D} \sim \zeta_{0} \rho \psi^{2} g^{2}(\sigma) N^{3}, \quad J_{\mathrm{e}} \sim N^{0} G_{N}^{-1}
\end{aligned}
$$

The $N$-scaling laws are identical to reptation theory ${ }^{2)}$. Note that PMC predictions obey the Fick's law type relation, $D \tau_{D} \sim R_{\mathrm{g}}^{2}$, as expected since a common relaxation process controls the diffusional and viscoelastic memory functions ${ }^{47)}$. A hand-waving derivation of these results follows from the fact that there are of order $N^{1 / 2}$ correlated contacts between a pair of entangled random coils in 3-dimensions, and the strength of the hard core force is proportional to $g(\sigma)$. Since the entanglement friction is proportional to a force-force time correlation, the net frictional enhancement might be argued to scale as $\left\{g(\sigma) N^{1 / 2}\right\}^{2}$. This argument immediately yields the above expression for $D$, and the relaxation time and shear viscosity follow from the Fick's law relation. Generalization to arbitrary spatial dimension, $d$, is easily achieved with the result ${ }^{39,47)}$

$$
\beta \zeta_{0} D=N^{-1}\left[1+b\left\{g(d) N^{\theta}\right\}^{2}\right]^{-1}, \quad \theta=2-(d / 2)
$$

Thus, entanglement effects are predicted to become irrelevant for $d>4$. This result clearly shows the different physical content of PMC theory compared to the cooperative cluster dynamics theory of Douglas and Hubbard ${ }^{18)}$ where Rouse behavior is recovered only in the limit $d \rightarrow \infty$.

Since the prefactors are predicted by PMC theory, property-dependent crossover degrees of polymerization are also determined and given by: $N_{D} \sim N_{t} \sim[g(\sigma)]^{-2}, N_{\eta}$ $\sim[g(\sigma) \psi]^{-1}$. Based on the PRISM theory results for Gaussian thread chains discussed in section IIB, one obtains the prediction that all crossover degrees of polymerization scale as $\left(\rho \sigma^{3}\right)^{-2}$. In good solvents, this $\rho^{-5 / 4}$ law is in good agreement with reptation plus scaling theory ${ }^{2,4)}$, while in theta solvents there are differences ${ }^{43}$. Indeed, the concentration scaling of transport coefficients in theta solvents remains rather poorly understood, and there are conflicting predictions within both reptation/ tube approaches and alternative theories ${ }^{4,43}$. 
The corresponding results based on the recent PMC analysis of Fuchs and Schweizer which relax the naive frozen matrix assumption are ${ }^{48)}$

$$
\begin{aligned}
& \frac{D}{D_{\mathrm{R}}}=\left(1+\lambda_{D} \frac{N}{N_{\mathrm{e}}}\right)^{-1}, \quad \frac{\eta}{\eta_{\mathrm{R}}}=1+\lambda_{\eta}\left(\frac{N}{N_{\mathrm{e}}}\right)^{2}, \\
& \frac{\tau_{D}}{\tau_{0}}=\frac{N^{3}}{\alpha N_{\mathrm{e}}}, \quad \frac{D \eta}{G_{N} R_{\mathrm{g}}^{2}}=\frac{3}{16} \\
& \lambda_{D}=\frac{32}{3 \alpha}, \quad \lambda_{\eta}=\frac{12}{\alpha}, \quad a^{-1}=\frac{g^{2}(\sigma) S_{0}}{2} \sim\left\langle F^{2}\right\rangle \rho^{-1}
\end{aligned}
$$

where the subscript " $\mathrm{R}$ " refers to the Rouse results. The $N$-dependence of these predictions are identical to the original PMC results ${ }^{37)}$ and reptation theory. The predicted "Stokes-Einstein ratio" of 3/16 is remarkably close to the corresponding tube model value of $5 / 24$. However, the material and density-dependent prefactors differ in some respects from those in Eq. (3.1). The experimentally defined $N_{\mathrm{e}}$ enters directly, and an entanglement strength parameter $\alpha$ enters as a fundamental material and concentration-dependent quantity, in contrast to reptation theory where it takes on the universal value of $a=32 / 9$. As discussed in section IV, this feature plays an important role when finite $N$ corrections are considered.

Based on the PRISM theory results for thread chains discussed in section IIB (accurate for semidilute solutions), one obtains the prediction that $\alpha$ is independent of polymer density, and hence all crossover degrees of polymerization are proportional to $N_{\mathrm{e}} \sim\left(\rho \sigma^{3}\right)^{-2}$ in complete agreement with the original PMC analysis of Eqs. (3.1). The corresponding polymer concentration dependences in theta $\left(\sigma \sim \rho^{0}\right)$ and good $\left(\sigma \sim \rho^{-1 / 8}\right)$ solutions were derived by Schweizer and Szamel ${ }^{43)}$

$$
\begin{aligned}
& \frac{\eta}{\zeta_{0}} \sim N^{3} \rho^{5} \sim\left(\rho / \rho^{*}\right)^{5} \sqrt{N}, \quad D \sim \rho^{-2} N^{-2}, \frac{\tau_{D}}{\tau_{0}} \sim N^{3} \rho^{2} \quad \text { Theta } \\
& \frac{\eta}{\zeta_{0}} \sim N^{3} \rho^{7 / 2} \sim\left(\rho / \rho^{*}\right)^{7 / 2} N^{1 / 5}, \quad D \sim \rho^{-5 / 4} N^{-2}, \frac{\tau_{D}}{\tau_{0}} \sim N^{3} \rho^{5 / 4} \text { Good }
\end{aligned}
$$

where $\rho^{*} \sim N / R_{\mathrm{g}}^{3}$ is the semidilute crossover concentration. These results agree with reptation/scaling behavior in good solvents, but there are significant differences in theta solvents ${ }^{43)}$. Whether Eq. (3.5) applies to concentrated and/or theta solutions is subtle due to the possible inadequacy of the Gaussian thread or string models employed in PRISM theory to obtain the equilibrium properties.

\section{B. Anomalous diffusion}

In the long chain limit, based on the RR projected dynamics, PMC theory predicts there are 5 distinct time regimes: early times $t \ll \tau_{\mathrm{C}}$ (Rouse dynamics), three intermediate regimes: $\tau_{\mathrm{C}} \ll t \ll \tau_{\mathrm{R}}, \tau_{\mathrm{R}} \ll t \ll \tau_{\mathrm{RR}}, \tau_{\mathrm{RR}} \ll t \ll \tau_{\mathrm{D}}$, and the terminal Markovian regime of free diffusion $t \gg \tau_{\mathrm{D}}$. Whether these distinct regimes are observable for finite $N$ is a subtle issue. 
Based on either slow variable choice, PMC theory predictions for the time dependence of the center-of-mass and segmental (chain-averaged) mean square displacements in the large $N$ limit are ${ }^{37,39)}$ :

$$
\begin{array}{rlrl}
\beta \zeta_{0}\left\langle\left(\boldsymbol{R}_{\mathrm{CM}}(t)-\boldsymbol{R}_{\mathrm{CM}}(0)\right)^{2}\right\rangle & \sim t / N & & \text { for } t \ll \tau_{\mathrm{C}} \\
& \sim t^{9 / 16} / N & & \text { for } \tau_{\mathrm{C}} \ll t \ll \tau_{\mathrm{R}} \\
& \sim t^{3 / 4} / N^{11 / 8} & & \text { for } \tau_{\mathrm{R}} \ll t \ll \tau_{\mathrm{RR}} \\
& \sim t / N^{2} & & \text { for } t \gg \tau_{\mathrm{RR}} \\
\beta \zeta_{0}\left\langle(\boldsymbol{r}(t)-\boldsymbol{r}(0))^{2}\right\rangle & \sim t^{1 / 2} & & \\
& \sim t^{9 / 32} / N^{1 / 4} & \text { for } t \ll \tau_{\mathrm{C}} \\
& \sim t^{3 / 8} / N^{7 / 16} & \text { for } \quad \tau_{\mathrm{C}} \ll t \ll \tau_{\mathrm{R}} \\
& \sim D t \sim t / N^{2} & \text { for } \quad \tau_{\mathrm{R}} \ll t \ll \tau_{\mathrm{RR}} \\
& & \text { for } t \gg \tau_{\mathrm{D}} \sim N^{3}
\end{array}
$$

The time scaling exponents for anomalous segmental diffusion in the first (widest) intermediate regime are in close, but not exact, agreement with the reptation/tube values of $1 / 2$ and $1 / 4$, respectively. However, in the final intermediate regime, $\tau_{\mathrm{R}} \ll t$ $\ll \tau_{\text {D }}$, reptation predicts $\mathrm{t}^{1}$ and $\mathrm{t}^{1 / 2}$ laws for $\mathrm{CM}$ and segmental diffusion, while PMC theory predicts this regime is bifurated with different exponents. Analytic results for segmental diffusion in the final intermediate regime, $\tau_{\mathrm{RR}} \ll t \ll \tau_{\mathrm{D}}$, are not easily derived due to the possible importance of both time-dependent memory functions $M(t)$ and $\Sigma(t)$. Existing computer simulations on lightly entangled chains cannot distinguish between the PMC and reptation predictions, and the later intermediate regimes are very difficult to observe ${ }^{14,31}$.

\section{Finite frequency response functions}

Spatially-unresolved $(k=0)$ response functions are expressable in terms of the linear normal mode time correlation functions. The (single chain) stress relaxation function is given by ${ }^{37}$ )

$$
\begin{aligned}
G(t)= & \frac{\beta \rho}{N} K_{\mathrm{S}}^{2} \int_{0}^{N} \mathrm{~d} a \int_{0}^{N} \mathrm{~d} \gamma\left\langle x_{a} x_{\gamma}(t)\right\rangle\left\langle\frac{\partial^{2} y_{\alpha}}{\partial \alpha^{2}} \frac{\partial^{2} y_{\gamma}(t)}{\partial \gamma^{2}}\right\rangle \\
& \sim N^{-3} \int_{1}^{\infty} \mathrm{d} p p^{4} C_{p}^{2}(t)
\end{aligned}
$$

which places heavy emphasis on the broad spectrum of internal chain normal mode contributions $1 \ll p \ll N$. The intermediate time domain predictions of PMC theory are given by ${ }^{40,41,47,48)}$

$$
\begin{aligned}
\frac{G(t)}{G_{N}} & =1+N^{-3 / 4}\left(\frac{\tau_{1}}{t}\right)^{9 / 32}-N^{-1}\left(\frac{t}{\tau_{2}}\right)^{3 / 16} & & \text { for } \quad \tau_{\mathrm{C}} \ll t \ll \tau_{\mathrm{R}} \\
& =1-N^{-9 / 8}\left(\frac{t}{\tau_{3}}\right)^{1 / 4} & & \text { for } \quad \tau_{\mathrm{R}} \ll t \ll \tau_{\mathrm{RR}}
\end{aligned}
$$


where $\tau_{j}$ are $N$-independent system-specific times. The first line describes the entanglement formation process via a fractional inverse power law approach to a transient plateau with an exponent and $\mathrm{N}$-dependent amplitude determined by anomalous segmental diffusion. As discussed in section VI, this contribution is not expected to be observable due to overlap with short time Rouse contributions. The plateau decays weakly in the characteristic $1-a t^{x}$ von Schweidler manner ${ }^{52)}$, with an exponent and $N$-dependent amplitude determined by the viscoelastic memory function. Physically, this weak decay corresponds to the disentanglement associated with the relaxation of constraining forces via polymer shape, or internal mode, fluctuations. The mathematical form of Eq. (3.9) bears a striking similiarity to mode-coupling theory predictions for the glass transition problem ${ }^{52}$. However, the PMC result is not of a precisely "dynamical scaling law form" as found from the self-consistent mode-coupling theory of the glass transition ${ }^{52)}$. Moreover, the underlying physics of the entanglement and glass formation problems are entirely different. For example, one consequence is that the time exponents are nonuniversal for the glass formation problem, but are predicted to be universal for entangled polymer dynamics. Essentially identical PMC predictions are obtained for the bond vector orientation function, $B(t)$ $=\langle\boldsymbol{b} \cdot \boldsymbol{b}(t)\rangle$, where $\boldsymbol{b}$ connects nearest neighbor bonded segments ${ }^{40,41,47,48}$.

It is important to appreciate that PMC theory has predicted, not assumed, a fundamental hallmark of entanglement dynamics: the near arrest of conformational and stress relaxation with the attendant rubbery network response, but continued mass transport in a slowed down, anomalous diffusion fashion. In this respect, PMC and reptation/tube theory seem similiar, although PMC theory invokes highly cooperative, but non-reptative, motions at the polymer trajectory level (see Fig. 4).

Eq. (3.9) has immediate consequences for pre-terminal $\left(t \ll \tau_{\mathrm{D}}\right)$ frequency domain measurements of storage and loss shear stress moduli. Based on an experimentallymotivated modification of Eq. (3.9a) which describes the early time decay onto the plateau as following a $t^{-1 / 2}$ Rouse law, one can derive ${ }^{41,47,48)}$

$$
\begin{aligned}
\omega_{\min } & \sim N^{-16 / 11}, G^{\prime \prime}\left(\omega_{\min }\right) \sim \tan \left(\delta_{\min }\right) \sim N^{-8 / 11} \\
G^{\prime \prime}(\omega) \sim \omega^{-3 / 16} & \text { for } \tau_{\mathrm{R}}^{-1}<\omega<\omega_{\min } \\
\sim \omega^{-1 / 4} & \text { for } \quad \tau_{\mathrm{RR}}^{-1}<\omega<\tau_{\mathrm{R}}^{-1}
\end{aligned}
$$

Here, $\omega_{\min }$ is the frequency at the loss modulus minimum separating Rouse-like from entangled dynamics, and $\tan \delta$ is the ratio of loss to storage moduli. The predicted $N$ and frequency scaling laws for the shear stress loss modulus are in excellent accord with experiments. For example, many years ago Ferry ${ }^{1)}$ found that the minimum value of the loss tangent scaled as an inverse power law with $N$ with an exponent of $\cong 0.7-0.8$. Winter and coworkers ${ }^{22)}$ have recently emphasized that even for ultra high molecular weight melts the pre-terminal loss modulus is a power law, $\omega^{-4}$, over $3-4$ orders of magnitude of frequency with $\Delta \cong 0.18-0.23$. This scaling behavior is in excellent accord with PMC theory, but very far from the simple reptation/tube theory ${ }^{2,3)}$ law of $\omega^{-0.5}$. Winter has constructed a successful phenomenological model based on a self-similiar distribution of relaxation times ${ }^{22)}$. For the pre- 
terminal regime, this model corresponds in the time domain to a $1-a t^{n}$ type decay of $G(t)$, where $n=0.22 \pm 0.02$ based on data fits for different polymers. PMC theory provides a fundamental theoretical basis for this self-similiar von Schweidler type behavior, and predicts exponents in very good accord with experiment. The physical origin within PMC theory is clear: continuous disentanglement (enhanced dissipation) associated with the relaxation of the viscoelastic memory function via polymer shape fluctuations. This process is not connected to the anomalous non-reptation scaling of the terminal time and viscosity, e.g., $N^{3.4}$, since our present analysis is based on asymptotic PMC theory which predicts the classic $N^{3}$ law. This feature is also in strong contrast to phenomenological extended tube models $3,4,23,24,71,72$ ) which invoke a direct connection, and do not provide a fundamental basis for a power law response of the form $\omega^{-0.2}$.

An illuminating relationship between PMC theory and the reptation/tube model for viscoelastic properties can be made by invoking an additional approximation. If one assumes $M(t)$ decays only via probe center-of-mass translation, i.e. if one ignores all internal mode shape fluctuation contributions to the single chain dynamic propagator so that $F^{\mathrm{R}}(k, t)=\exp \left(-k^{2} D_{\mathrm{RR}} t\right)$, then Eq. (2.28) for $M(t)$ on intermediate time scales is dramatically modified. For $k R_{\mathrm{g}}>1$ and $t<\tau_{\mathrm{RR}}$ one obtains

$$
M(t) \sim \sqrt{N}-a \sqrt{D_{\mathrm{RR}}} t^{1 / 2} \Rightarrow G^{\prime \prime}(\omega) \sim\left(N^{3} \omega\right)^{-1 / 2}
$$

This prediction for the pre-terminal loss modulus is identical to the pure reptation/ tube theory result ${ }^{2,3)}$. In a sense this seems consistent, since assuming within PMC theory that only coherent probe center-of-mass motion can relax the entanglement forces is roughly akin to the tube model assumption that disentanglement only occurs via the coherent single chain reptative motion. However, this assumption misses the dominant mechanism for entanglement relaxation associated with probe shape fluctuations which are the origin of the greatly enhanced dissipation and much shallower slope of the loss modulus. This assumption would also radically change the power law time dependence of the $\Sigma(t)$ memory function, and hence the PMC predictions for center-of-mass and segmental anomalous diffusion and dielectric relaxation (see below). However, ignoring the coil shape fluctuations does not change the asymptotic $N$-scaling law for the viscosity determined by the Markovian limit of the memory functions.

Predictions for the end-to-end vector time correlation function, $\langle\boldsymbol{R}(0) \cdot \boldsymbol{R}(t)\rangle=$ $16 \Sigma_{p=\text { odd }} C_{p}(t)$, which is generally identified with the measured dielectric response, are given in the intermediate time domain by ${ }^{47,48)}$

$$
\begin{aligned}
\frac{P(t)}{P(0)} \equiv\langle\boldsymbol{R} \cdot \boldsymbol{R}(t)\rangle /\left\langle R^{2}\right\rangle & =1-N^{-5 / 4}\left(t / \tau_{4}\right)^{9 / 32} \quad \text { for } \quad \tau_{\mathrm{C}} \ll t \ll \tau_{\mathrm{R}} \\
& =1-N^{-23 / 16}\left(t / \tau_{5}\right)^{3 / 8} \quad \text { for } \quad \tau_{\mathrm{R}} \ll t \ll \tau_{\mathrm{RR}}
\end{aligned}
$$

where $\tau_{j}$ are $N$-independent, system-specific times. The von Schweidler fractional time dependence ${ }^{52)}$ is again predicted, but with exponents and $N$-dependent amplitudes significantly different than for stress relaxation. The reason for this is dielectric 
response is dominated by the large scale motions (small $p$ modes), in strong contrast with $G(t)$. It is the fractional power law dependence of the diffusional memory function, $\Sigma(t)$, which plays a central role, not $M(t)$ as was true for the stress relaxation modulus. The terminal dielectric relaxation time is related to the observable stress relaxation time as $\tau_{\mathrm{di}} \cong b \tau_{\mathrm{D}}$, where ${ }^{47,48)} b \cong 2-2.6$.

The pre-terminal relaxation frequency domain predictions for the dielectric loss $\operatorname{are}^{47,48)}$

$$
\begin{aligned}
\varepsilon^{\prime \prime}(\omega) & \sim N^{-5 / 4} \omega^{-9 / 32} & \text { for } & \tau_{\mathrm{R}}^{-1}<\omega<\tau_{\mathrm{C}}^{-1} \\
& \sim N^{-23 / 16} \omega^{-3 / 8} & \text { for } & \tau_{\mathrm{RR}}^{-1}<\omega<\tau_{\mathbf{R}}^{-1}
\end{aligned}
$$

The predicted exponents of the $\omega^{-\Delta}$ frequency scaling law are again much smaller than the reptation value of $\Delta=0.5$ (which is identical to the $G^{\prime \prime}(\omega)$ case). Moreover, the magnitude of the PMC theory exponents, $\Delta=0.28-0.38$, is in agreement with dielectric measurements of Adachi, Kotada and coworkers ${ }^{20)}$. The fact that the dielectric loss exponents (frequency and $N$ scalings) are significantly larger than their shear stress counterparts, implies the dielectric loss is considerably narrower than the stress loss modulus. This result is also in agreement with experiment ${ }^{20)}$.

We again emphasize that the PMC prediction of non-reptation frequency power laws is not connected with the anomalous non-reptation scaling with $N$ of the stress or dielectric relaxation time. Recent dielectric probe experiments ${ }^{20)}$ in heavily entangled nonpolar matrices appear to have dramatically confirmed the decoupling of the non-reptative $N$ and frequency scalings. A classic $N^{3}$ law was observed for the probe dielectric relaxation time, but the loss modulus was still very broad and qualitatively identical to the one-component melt behavior. The authors also concluded that collective matrix relaxation, or constraint release (ignored in our present asymptotic analysis), is the physical origin of the anomalous $N$-scaling of the terminal relaxation time ${ }^{20)}$. This idea is supported by recent PMC work described in section IV.

Predictions for other dynamic response functions, such as nonexponential decay of $C_{p}(t)$, and field cycling NMR measurements of the spin-lattice relaxation time $T_{1}(\omega)$, can also be addressed.

\section{Spatially-resolved correlation functions}

Spatially-resolved experimental probes of entangled dynamics have recently been developed. Neutron Spin Echo (NSE) is a time domain spectroscopy which measures the coherent, single chain dynamic structure factor at relatively short times as a function of wavevector ${ }^{73)}$. Pulsed-field-gradient nuclear magnetic resonance (PFG-NMR) probes the incoherent single segment dynamic structure factor on (late) intermediate and long time/length scales ${ }^{74)}$. The relevant time correlation functions are 


$$
\begin{aligned}
& \omega_{\mathrm{inc}}(k, t)=N^{-1} \int_{0}^{N} \mathrm{~d} \alpha\left\langle\mathrm{e}^{\mathrm{i} \boldsymbol{k} \cdot\left(\boldsymbol{r}_{a}-\boldsymbol{r}_{a}(t)\right)}\right\rangle \\
& \omega_{\mathrm{coh}}(k, t)=N^{-2} \int_{0}^{N} \mathrm{~d} \alpha \int_{0}^{N} \mathrm{~d} \gamma\left\langle\mathrm{e}^{\mathrm{i} \boldsymbol{k} \cdot\left(r_{a}-r_{7}(t)\right)}\right\rangle
\end{aligned}
$$

For a stochastic Gaussian process (as invoked for tractability reasons by PMC theory), the simple second cumulant expansion forms apply ${ }^{47}$ )

$$
\omega_{\mathrm{coh}}(k, t) \cong \omega_{\mathrm{inc}}(k, t)=\exp \left(-k^{2}\left\langle(\boldsymbol{r}-\boldsymbol{r}(t))^{2}\right\rangle / 6\right)
$$

Thus, PMC theory predictions follow from the segmental diffusion results described in section IIIB. Recent incoherent experiments seem to support the Gaussian wavevector dependence ${ }^{74)}$. Time dependences of an apparent diffusion coefficient have then been defined

$$
D_{\text {app }}(t) \equiv\left(t k^{2}\right)^{-1} \ln \left\{\omega_{\text {inc }}(k, t)\right\}
$$

In contrast, reptation theory predicts ${ }^{2,3)}$ strongly non-Gaussian forms (power law $k$ and $t$ dependences), in apparent disagreement with PFG-NMR experiments ${ }^{74)}$. However, as emphasized by Fatkullin and Kimmich, more careful analysis, and data at larger $k$ and smaller times, are required to draw definitive conclusions, although these authors also conclude reptation theory is in poor agreement with their experiments ${ }^{75)}$.

Coherent NSE experiments by Richter and coworkers have observed distinctive $k$-dependent crossovers from Rouse to entangled response, including near plateau behavior ${ }^{73)}$. Unfortunately, the data is restricted to short times and is often rather noisy. Thus, disparate theoretical approaches (tube-based and non-tube theories) have been shown to provide good fits to the data ${ }^{15,28,69,73,76)}$. We believe this fact renders existing NSE data (and also computer simulations) inadequate for purposes of definitively testing the fundamental validity of reptation/tube and alternative theories. It is particularly significant to note that the stochastic, non-tube theory of Chatterjee and Loring ${ }^{76)}$ is in very good agreement with NSE data, despite the fact that the calculation is based on a Gaussian approximation to the coherent structure factor. The latter aspect implies no rigorous plateau is present (since segments never literally localize in space), and terminal relaxation is highly $k$-dependent ${ }^{76)}$. These features are in contradiction with reptation theory ${ }^{2,3)}$. Unfortunately, experimental limitations preclude NSE spectroscopy from probing the long time regime.

PMC theory predictions for the coherent dynamic structure factor are qualitatively similiar to the stochastic dynamics approach ${ }^{47,77)}$. Prior applications of the RR theory to study entanglement onset, and the $p$-dependence of the Rouse mode relaxation times, have been favorably compared ${ }^{42)}$ at a qualitative level with NSE measurements ${ }^{73)}$. We caution that the linear nature of the GLE's may be inadequate for describing spatially resolved dynamic structure factors, and alternative approaches within the Mori-Zwanzig framework are presently under study. 


\section{Finite $N$ corrections in solutions and melts}

As sketched in the Introduction, there are many discrepancies between the simplest version of reptation/tube theory and experiments in solutions, melts, and gels. As discussed in section III, many of the pre-terminal finite frequency anomalies appear to be qualitatively resolved by PMC theory. However, the $N$-dependence of many of the transport coefficients and relaxation times remains a mystery. The anomalies appear to be very different in solution (or gel) and melt states, and also for translational. diffusion versus shear viscosity and chain relaxation times. We believe a fundamental, non-phenomenological approach is required to convincingly understand all of these anomalies within a common theoretical framework based on a minimum number of new parameters. Here we describe a recent attempt by Fuchs and Schweizer to analytically formulate and apply such a theory within the PMC framework $^{48)}$. The finite size dynamical corrections are connected with physicallymeaningful equilibrium structural and dynamic properties.

\section{A. Constraint porosity and self-consistent constraint release}

The extended tube-based approaches invoke additional non-reptative relaxation or transport mechanisms at the chain trajectory level in a generally statistically independent fashion. The various non-asymptotic anomalies in diffusion and viscosity are generally treated on a case-by-case basis, employing distinct ad hoc assumptions. In strong contrast, within microscopic PMC theory the fluctuating force memory functions are the fundamental objects for all dynamic properties. The finite $N$ weakening of these entanglement force time correlations via either amplitude or "porosity" of constraints, or accelerated relaxation due to collective "self-consistent constraint release", was discussed as points (1) and (2) in section IID. A schematic of the relevant length scales which are important for finite size corrections is shown in Fig. 2.

The most general form of the zero frequency memory functions, which determine the entanglement corrections to the transport coefficients and relaxation times, follows from Eqs. (2.29) and (2.34)-(2.36). The self-consistent equation for the terminal chain relaxation time for $N>N_{\mathrm{e}}$ is ${ }^{48)}$

$$
\begin{aligned}
& 2 \tau_{\mathrm{D}}= \frac{\beta \sigma^{2}}{6} \int_{0}^{\infty} \mathrm{d} t M(t)=\left(12 \pi^{2} \rho\right)^{-1} \frac{N^{2}}{N_{\mathrm{e}}} \int_{0}^{\infty} \mathrm{d} k k^{2} \cdot \\
& {\left[1+\left(k \xi_{\mathrm{c}}\right)^{2}\right]^{-2} \frac{\exp \left(-k^{2} r_{\mathrm{c}}^{2} / 36\right)}{1+\left(k \xi_{\rho}\right)^{2}} \cdot \frac{1}{\tau_{\mathrm{D}}^{-1}+\frac{k^{2}\left(1+k^{2} \xi_{\mathrm{c}}^{2}\right)}{\beta\left(\zeta_{0}+\Delta \zeta\right) N}} } \\
& \frac{\tau_{\mathrm{D}}}{\tau_{0}} \equiv a^{-1} \frac{N^{3}}{N_{\mathrm{e}}} K_{\tau}\left(N / N_{\mathrm{e}}, a, \delta \equiv \xi_{\rho} / r_{\mathrm{c}}\right)
\end{aligned}
$$

In the second line, $\Delta \zeta$ is the RR theory friction constant given by Eqs. (2.11)(2.13), and the (large $N$ ) equilibrium $k=0$ PRISM relation ${ }^{57)}-\rho C_{0} S_{0}=1$ has been utilized. The final expression in Eq. (4.1) has been written in a form designed to display the large $N$ asymptotic result arising from power law counting based on the cor- 
relation hole length scale $\xi_{\mathrm{c}}=R_{\mathrm{g}} / 2^{1 / 2}$, which includes the entanglement strength parameter $a$ of Eq. (3.4). The large $N$ result is multiplied by a function $K_{\tau}$ which depends on reduced degree of polymerization and nonuniversal intrinsic length scales: the physical and entanglement mesh lengths $\xi_{\rho}$ and $r_{\mathrm{c}}$, respectively. This function describes the finite size "constraint porosity" corrections to the amplitudes on a length scale $2 \pi / k$ via the length scale ratio $\delta=\xi_{\rho} / r_{\mathrm{c}}$ which is effectively set equal to zero in the reptation/tube theory. This correction function also depends on the terminal relaxation time associated with the self-consistently determined "constraint release" effect. The latter correction is controlled by the time scale ratio $\tau_{\mathrm{RR}} l$ $\tau_{D} \sim\left(N_{\mathrm{e}} / N\right)^{1 / 2}$ for very long chains ${ }^{48)}$. However, as shown below, this ratio is apparently not generally small for experimental degrees of polymerization. The implicitly defined function $K_{\tau}$ approaches unity in the $N / N_{\mathrm{e}} \rightarrow \infty$ limit.

The shear viscosity is given in terms of its Rouse value by ${ }^{48)}$

$$
\eta=\eta_{\mathrm{R}}\left[1+\frac{12}{a}\left(\frac{N}{N_{\mathrm{e}}}\right)^{2} K_{\tau}\left(N / N_{\mathrm{e}}, a, \delta\right)\right]
$$

Because the viscosity and conformational disentanglement process are dominated by macromolecular scale dynamical correlations $\left(k R_{\mathrm{g}}\right.$ of order unity in Eq. (4.1); see Fig. 3), the porosity corrections are predicted to play very little role for viscoelastic properties. Thus, deviations from the $N^{3}$ law will be dominated by the self-consistent constraint release process which is sensitive only to reduced degree of polymerization $N / N_{\mathrm{e}}$, and not polymer concentration explicitly. One thereby expects deviations from the $N^{3}$ law will emerge in a nearly identical fashion under all solution and melt conditions, a trend consistent with experimental observations ${ }^{1)}$.

The entanglement friction constant enhancement, $-1+D_{\mathrm{R}} / D$, is given by ${ }^{48)}$

$$
\begin{aligned}
& \int_{0}^{\infty} \mathrm{d} t \frac{\Sigma(t)}{\zeta_{0}}= \\
& \left(6 \pi^{2} \beta \zeta_{0} \rho\right)^{-1} \frac{N}{N_{\mathrm{e}}} \int_{0}^{\infty} \mathrm{d} k k^{4}\left[1+\left(k \xi_{\mathrm{c}}\right)^{2}\right]^{-1} \frac{\exp \left(-k^{2} r_{c}^{2} / 36\right)}{1+\left(k \xi_{\mathrm{p}}\right)^{2}} \cdot \frac{1}{\tau_{\mathrm{D}}^{-1}+\frac{k^{2}\left(1+k^{2} \xi_{\mathrm{c}}^{2}\right)}{\beta\left(\zeta_{0}+\Delta \zeta\right) N}} \\
& \equiv \frac{32}{3 \alpha} \frac{N}{N_{\mathrm{e}}} K_{\Sigma}\left(N / N_{\mathrm{e}}, \alpha, \delta\right)
\end{aligned}
$$

Again, the asymptotic result is explicitly displayed, and the correction function $K_{\Sigma}$ is implicitly defined. As emphasized in Fig. 3, self-diffusion is much more sensitive to entanglement force correlations (via the amplitude factors) on smaller length scales (higher $k$ ) than the viscoelastic memory function. Thus, PMC theory predicts the non-diffusive collective constraint release process will have a much smaller effect on self and tracer diffusion than for the viscosity and chain relaxation time. However, even in the frozen matrix limit, a condition realizable in tracer experiments, the asymptotic behavior is not necessarily recovered under experimentally realizable conditions due to the constraint porosity effects associated with finite screening and entanglement mesh length scales. Since the latter are dependent on 
chemical structure, concentration and solvent quality, in contrast with the finite size viscosity corrections a range of possible apparent scaling laws are expected to emerge.

Both constraint porosity and constraint release corrections reduce friction, and hence speed up motion, in a manner which is enhanced as the chains become shorter. Thus, if apparent $N$-scaling laws emerge one generally expects the effective exponents will be larger than the asymptotic values. This effect is not described by postulating alternative processes at the chain trajectory level. Rather, it arises from additional relaxation channels and/or weakening of the spatially-resolved entanglement constraints by dynamic and static meshes, as described at the fundamental level of the entanglement force time correlations. As a matter of principle, constraint porosity and constraint release effects are neither additive nor separable. Thus, the net diffusion constant cannot be expressed as a contribution from different transport mechansims as done in phenomenological tube model approaches.

Explicit expressions for the dielectric relaxation time, and the effective friction constant for the tracer diffusion problem (chemically identical tracer degree of polymerization $N$ not equal to matrix chain length $P$ ), have also been derived ${ }^{48)}$.

\section{B. Predicted trends and model calculations}

Predictions expressed as a function of $N / N_{\mathrm{e}}$ require specification of the two dimensionless material parameters of clear physical meaning: $\alpha$ and $\delta=\xi_{\rho} / r_{\mathrm{c}}$. The latter ratio parameter can be obtained by direct experimental measurements. Estimates for melts and solutions can be made based on experimental properties such as those given in the Tab. 1 . One finds for the nearly incompressible melt state, $\delta \cong$ $0.03 \pm 0.02$. However, in good solvents much larger values are obtained $\delta \cong 0.3 \pm$

Tab. 1. Materials parameters of the different polymer melt systems studied and the three polystyrene solutions of 13,18 , and $40 \mathrm{wt} .-\%$. Listed are experimental values for the entanglement degree of polymerization (based on monomer mass) and crossover length $^{65)}$ (at $T=413 \mathrm{~K}$ ), $N_{\mathrm{f}}$ and $b=r_{\mathrm{c}}$, and the "packing length" $p=\left(\rho \sigma^{2}\right)^{-1} \cong \xi_{p}=$ melt density screening length ${ }^{65}$. The inverse interaction strength parameter, $\alpha$, and length scale ratio parameter, $\delta$, determined by fits of the theory to experiment are also listed

\begin{tabular}{l|ccccccccc} 
& PBD & PBDh & PDMS & PEO & PI & PS & PS(13) & PS (18) & PS(40) \\
\hline$N_{\mathrm{e}_{\mathrm{o}}}$ & 34 & 44 & 162 & 50 & 74 & 173 & 1250 & 923 & 407 \\
$b / \AA$ & 44 & 34 & 79 & 38 & 62 & 77 & & & \\
$\xi_{\rho} / \AA$ & 2.3 & 1.8 & 4.1 & 1.9 & 3.2 & 4.0 & & & \\
$\alpha$ & $4.9^{\mathrm{a})}$ & $4.3^{\mathrm{c})}$ & $2.8^{\mathrm{b})}$ & $2.6^{\mathrm{b})}$ & $2.2^{\mathrm{b})}$ & $2.2^{\mathrm{b})}$ & $4.0^{\mathrm{a})}$ & $2.8^{\mathrm{b})}$ & $2.5^{\mathrm{a})}$ \\
& $3.3^{\mathrm{b})}$ & & & & $3.5^{\mathrm{d})}$ & & $2.8^{\mathrm{b})}$ & & $2.8^{\mathrm{b})}$ \\
$\delta$ & 0.01 & 0.03 & 0.0 & 0.05 & 0.05 & 0.05 & 0.4 & 0.3 & 0.4
\end{tabular}

a) From viscosity.

b) From diffusion.

c) From viscosity and diffusion.

d) From dielectric relaxation time. 
0.1 , consistent with simulation studies of semidilute (lightly) entangled solutions ${ }^{78)}$. Based on experiments and scaling theory, $\delta$ is expected to be nonuniversal but concentration independent in semidilute solutions ${ }^{70)}$. Even larger values of $\delta$ are expected in theta solvents, which increase with dilution, since the density screening length increases more rapidily with $\rho$ than the corresponding entanglement mesh size under theta conditions ${ }^{70}$ ).

The force strength parameter $\alpha$ requires estimation from theory or simulation. Reptation theory predicts $\alpha=32 / 9 \cong 3.5$ corresponding to $\lambda_{D}=3$ in Eq. (3.4). Estimates based on analytic and numerical PRISM theory suggest melt values in the range $\alpha \cong 2-4$, and somewhat smaller values (larger $\left\langle F^{2}\right\rangle / \rho$ ) in semidilute and concentrated solutions ${ }^{48}$.

The above a priori parameter estimates are used as guides to explore the nature of the finite size corrections predicted by PMC theory. We begin with the effect of selfconsistent constraint release on the viscosity. Representative results are shown in Fig. 5. Only (seemingly) unrealistically large values of $\delta$ significantly modify the pure constraint release $(\delta=0)$ behavior. Although Eq. (4.1) does not predict a rigorous power law except in the asymptotic $\eta \sim N^{3}$ limit, an excellent effective power law over two orders of magnitude in $N$ does emerge with typical exponents in the range of $3.4 \pm 0.1$. The PMC theory conclusion that the physical mechanism of the "3.4 law" is constraint release is apparently in strong disagreement with essentially all the diverse reptation/tube approaches based on "contour fluctuations" "reptons"24), "tube dilation/plastization"26), "non-Fickian motion of chain ends""71) or other phenomenological concepts. However, matrix constraint release as the key physical mechanism is consistent with: (a) the recent tracer dielectric measurements

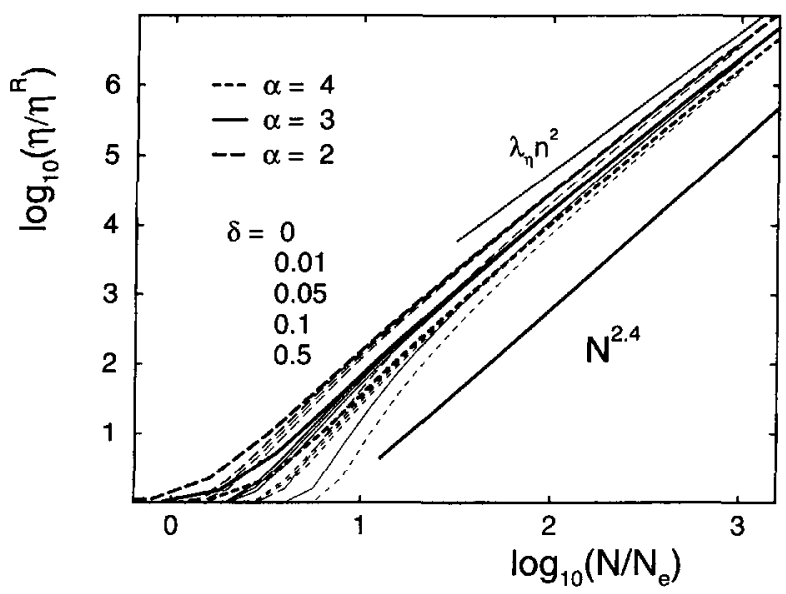

Fig. 5. Ratios of shear viscosity to Rouse viscosity for different physically-relevant values of the entanglement strength parameter $a$ and length scale ratio $\delta=\xi_{p} / r_{\mathrm{c}}$. Bold lines denote results neglecting constraint porosity for different $\alpha$, and thin lines are for fixed $\alpha$ with $\delta$ increasing from left to right. Lines of the same style are associated with a unique value of $\alpha$. A $N^{2.4}$ power law is shown for comparison. The asymptotic limiting behavior, $\lambda_{\eta} \cdot n^{2}$ where $n=N / N_{\mathrm{e}}$, is drawn as a thin dotted line for $\alpha=2$ or $\lambda_{\eta}=6$ 
of Adachi et al. ${ }^{20)}$, (b) the experimental insensitivity of the viscosity exponent to polymer concentration ${ }^{1,5)}$, and (c) a recent computer simulation study of Termonia $^{79)}$.

The extremely slow approach of the viscosity to its asymptotic $N^{3}$ law occurs because PMC theory predicts the leading correction is of the form: $\eta \sim N^{3}\left[1-c\left(N_{\mathrm{e}} /\right.\right.$ $N)^{1 / 4}$, where $c=(4 / 3) \alpha^{1 / 2}$. Even for values of $N / N_{\mathrm{e}}=1000$, the reduced viscosity is only roughly $70 \%$ of what is expected based on asymptotic analysis ${ }^{48)}$. This behavior is quite different than contour fluctuation and related tube-based approaches which predict a result of the form ${ }^{23)} \eta \sim N^{3}\left[1-a\left(N_{\mathrm{e}} / N\right)^{1 / 2}\right]^{3}$, where $\left(N_{\mathrm{e}} / N\right)^{1 / 2}$ is the control variable for finite size corrections. This difference is directly related to the idea that the (projected) fluctuating forces decay on the RR time scale.

The effect of constraint porosity can be most clearly seen for the problem of tracer diffusion of chains in a matrix of much longer chains $P \gg N$, i.e. the effectively frozen matrix limit. Model calculations for the tracer diffusion constant as a function of $N$ are shown in Fig. 6 for variable $\delta$ and two extreme choices of $a$ ( 3 for melts, and 0.5 which is relevant, perhaps, for solutions). In agreement with experiments ${ }^{80}$, for the melt-like case a very rapid, smooth crossover from the Rouse law to the asymptotic PMC (and reptation) scaling is found for small values of $\delta$ appropriate to the melt. However, for the second solution-like case, a non-asymptotic scaling regime emerges which persists out to $N / N_{\mathrm{e}} \cong 50-100$. The tracer diffusion constant lies appreciably above its asymptote. Apparent tracer diffusion exponents, $D_{\mathrm{tr}} \sim N^{-\nu}$, in the range $v=2.3-2.8$ seem possible. Crossover to the true $N^{-2}$ asymptote occurs at much larger $N / N_{\mathrm{e}}$ than in the melt case.

The above predictions are in general accord with tracer measurements in both semidilute and concentrated good solvent solutions ${ }^{81)}$, the understanding of which has remained a mystery ${ }^{21)}$. The underlying physical mechanism which generates non-melt-like scaling in solutions has no analog in any extended reptation/tube approaches we are aware of. Moreover, the PMC mechanism of constraint porosity

Fig. 6. Dimensionless tracer diffusion coefficients in dynamically frozen matrices. Two sets of curves are shown corresponding to different values of the asymptotic prefactor, $\lambda_{D}$, and corresponding to the upper or lower horizontal scale. Increasing steepness in the intermediate $N$ region occurs with increasing $\delta=$ $\xi_{p} / r_{\mathrm{c}}=0.01,0.05,0.1$, $0.2,0.3$, and 0.5 , respectively. Three power laws with arbitrary prefactors are shown for comparison

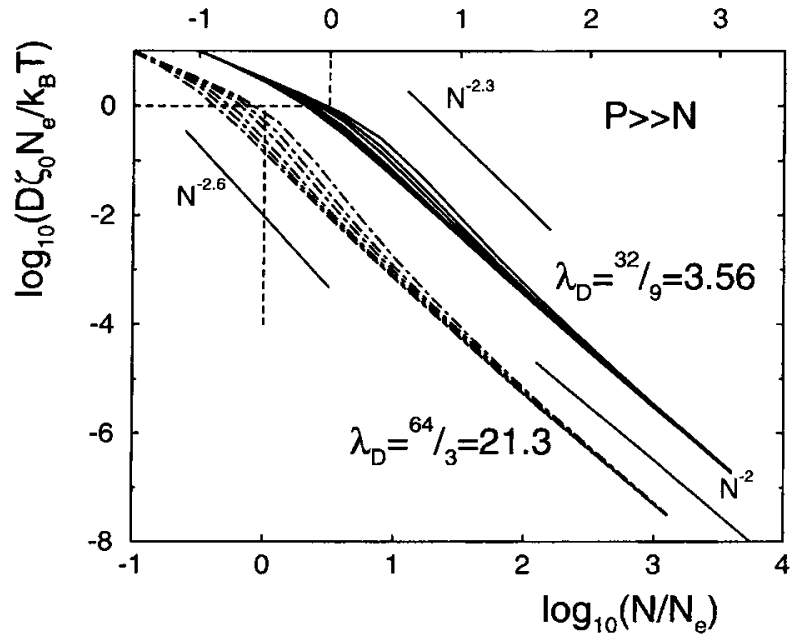


does not seem closely related to the qualitative "entropic barrier" ideas of Muthukumar and Baumgartner ${ }^{82,83)}$. The latter focus on the crossover case where the mesh size is comparable to the tracer radius-of-gyration. This condition is definitely not true for most solution conditions where $R_{\mathrm{g}} / \xi_{\rho} \gg 1$. The non-reptation diffusion constant exponents also do not have the same physical origin as the shear viscosity 3.4 exponent within PMC theory. This conclusion is in strong contrast with the presumed connection in solutions (but not melts) suggested by Rubinstein and Obukhov $^{84)}$. It appears that a microscopic approach relating liquid structure and friction is required to properly identify the large finite size corrections arising from spatial correlations of the matrix entanglement constraints. Chemical structure, solution density, and solvent quality dependences are expected for these corrections ${ }^{48)}$.

An example of model calculations relevant to the tracer diffusion and constraint release problem is shown in Fig. 7 for solution-like parameters. The predicted behavior is in qualitative agreement with experiments, and also with the various distinct, but tube-based, constraint release ideas of Graessley ${ }^{4)}, \mathrm{Klein}^{85}$, and others. However, PMC theory does not predict a simple additive form for the tracer diffusion constant as do phenomenological tube theories. For example, the Graessley result is $D_{\text {tr }} \sim N_{\mathrm{e}} N^{-2}\left\{1+\alpha_{\mathrm{cr}} N_{\mathrm{e}}^{2} N P^{-3}\right\}$ where $\alpha_{\mathrm{cr}}$ is an empirical parameter ${ }^{4)}$. For the solution case shown in Fig. 7, constraint porosity effects are also not negligible, although they become so under melt-like conditions ${ }^{48)}$. Further complications and diversity of possible experimental behaviors are expected when the tracer and matrix are not chemically identical.

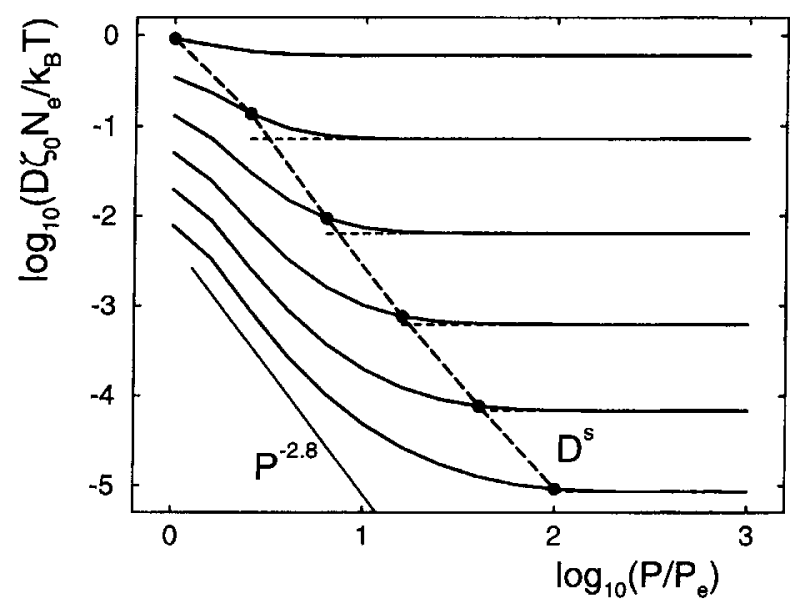

Fig. 7. Tracer diffusion coefficients in a polymer solution as a function of reduced matrix degree of polymerization for different degrees of tracer entanglement $N / N_{\mathrm{e}}$. $\log _{10}\left(N / N_{\mathrm{e}}\right)=0,0.4,0.8,1.2,1.6$, and 2 from top to bottom, and the solution-like parameters $\alpha=3, \delta=0.3, \lambda_{D}=18$ are employed. Self diffusion constants $D^{\mathrm{s}}$ (circles and long dashed), the asymptotic value $D^{\mathrm{tr}}$ (short horizontal dashed) for $P \rightarrow \infty$, and a $P^{-2.8}$ power law, are also shown 
Model calculations for the dependence of self and tracer dielectric times on matrix molecular weight have been performed, and qualitative agreement of the predicted trends with experiments is found ${ }^{48)}$.

In summary, it appears PMC theory with self-consistent constraint release and (multiple) porosity corrections at the level of the entanglement force dynamic correlations is able to account for all the puzzling non-asymptotic behaviors of transport coefficient and relaxation time scalings in melts and solutions. Description of diffusion and viscoelastic "anomalies" has been achieved in a unified manner based on structural parameters of clear microscopic meaning. The physical origin of the strong finite size corrections are predicted to be very different for translational diffusion versus shear viscosity and conformational relaxation. The key aspect of the theory is its spatial and temporal resolution of the amplitude and relaxation of entanglement forces. Phenomenological guesses for alternative real space motions are not invoked, although this also represents a limitation in the sense that an intuitive picture at the chain trajectory level is not readily deduced. We now turn to a discussion of quantitative applications to specific experimental studies.

\section{Experimental comparisons}

Quantitative applications of PMC theory to viscosity, self and tracer diffusion, and dielectric relaxation in entangled solutions and melts have been recently carried out by Fuchs and Schweizer ${ }^{48)}$. Here we summarize some of this work. Relevant material properties are collected in Tab. 1.

Study of the reduced melt viscosity versus reduced degree of polymerization highlights the effects of self-consistent constraint release. Only one fit parameter enters, the inverse entanglement force strength $\alpha$, since constraint porosity has little consequences on viscosity as discussed above. Fig. 8 shows data for polybutadiene (PBD) melts ${ }^{86)}$ out to ultra high molecular weights. PMC theory describes the data quite well based on the reasonable value of $\alpha=7.7$. A second fit is also shown which incorporates corrections of the data for the monomeric friction constant as discussed by Roovers $^{87)}$ and $\mathrm{O}^{\prime}$ Connor and Ball ${ }^{71)}$. Quantitative agreement is obtained, with $\alpha$ even closer to the reptation theory value of 3 . As more clearly seen in the inset, note that the asymptotic $N^{3}$ scaling is not yet exhibited by the data or PMC theory even though $N / N_{\mathrm{e}}>1000$.

Of special interest is the PMC theory prediction of a close connection between center-of-mass and conformational/stress relaxation dynamics. The combined diffusion and viscosity measurements of Pearson and coworkers ${ }^{88)}$ on the same hydrogenated PBD (PBDh) sample provide an excellent test of the theory. Fig. 9 shows the data and the PMC results. The viscosity fit fixes the nonuniversal parameter $a$, and therefore the constraint release contribution to $D$ and $\eta$. A value of $\alpha=5.2$ yields the best fit, which is reassuringly close to that extracted from the PBD analysis in Fig. 8. The experimental apparent power law $\eta \sim N^{3.47}$ is again well predicted by PMC theory, and explained as a finite size crossover effect due to the self-consistent constraint release mechanism.

A slightly smaller value of $a=4.3$ provides the best simultaneous fit of PMC theory to the self-diffusion and viscosity data of PBDh. The self-diffusion coefficient 


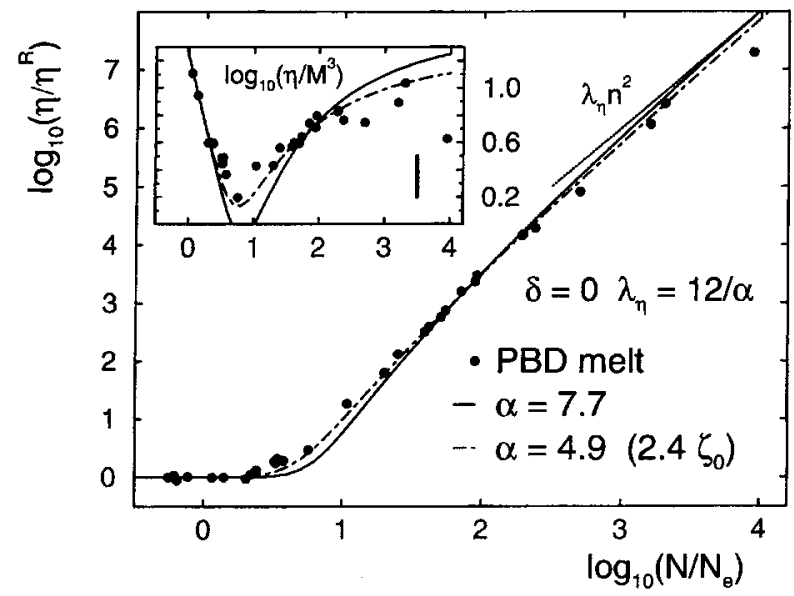

Fig. 8. Viscosity of PBD melt ${ }^{86)}$ reduced by its Rouse value; the solid circles are the experimental data. The solid line (chain curve) is a one-parameter PMC theory fit of the raw $^{86)}$ (local friction corrected ${ }^{71,87)}$ ) data. The thin dotted line is the asymptote for the second fit. The inset shows a logarithmic plot of the viscosity divided by the asymptotic scaling law factor (vertically shifted by a factor of 10 ) versus reduced degree of polymerization. The lines correspond to the same fits shown in the main figure. A vertical bar denotes the error bar reported in ref. ${ }^{86)}$

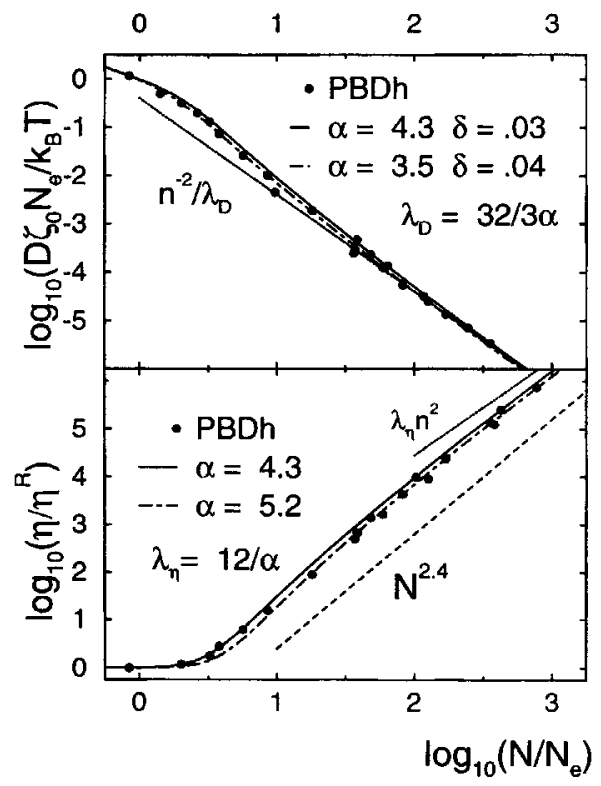

Fig. 9. Reduced self-diffusion constant and shear viscosity versus reduced molecular weight for a hydrogenated polybutadiene melt $(\mathrm{PBDh})^{88)}$. The solid lines are PMC theory simultaneous fits of $D$ and $\eta$ with a common value of $\alpha=$ 4.3 , and a $\delta=0.03$ from the diffusion fit. The dotted lines are the predicted asymptotic behaviors for the $\alpha=4.3$ fit. The chain curves show the best independent fits of the two data sets leading to slightly different values of fit parameters. The $N^{2.4}$ power law is shown for comparison 
"overshoots" the large $N$ asymptote, due primarily to constraint release since constraint porosity corrections are small in dense melts. Thus, based on the data comparisons and PMC theory we conclude that in dense melts constraint release is the dominant finite size effect, in agreement with our general arguments given in section B.

The dielectric relaxation time, $\tau_{\varepsilon}$, has also been studied ${ }^{48)}$. Model calculations show for melt-like parameters a power law scaling of $\tau_{\varepsilon} \sim N^{3.4 \pm 0.1}$ is predicted in an intermediate molecular weight window of $3<N / N_{\mathrm{e}}<100$. Fig. 10 shows a quantitative comparison with polyisoprene (PI) melt data ${ }^{20)}$ where $\delta=0.05$ was a priori fixed at a typical melt value. PMC predictions for two choices of $\alpha$ are shown, with $\alpha=$ 3.5 corresponding to a best fit value, while $\alpha=2.2$ was independently determined (although with large uncertainties due to experimental error bars) from fits to PI melt self-diffusion data (see Tab. 1 and Fig. 11). Good agreement between theory and experiment is found with $\alpha$ falling in the a priori expected range. We conclude that the non-reptation scaling with $N$ of the dielectric relaxation time is also due to self-consistent constraint release effects.

Fig. 10. Dielectric relaxation times for polyisoprene (PI) melts ${ }^{20)}$ versus reduced molecular weight. A melt-like value of $\delta=$ 0.05 is a priori selected, and theoretical results for two values of $a$ are shown

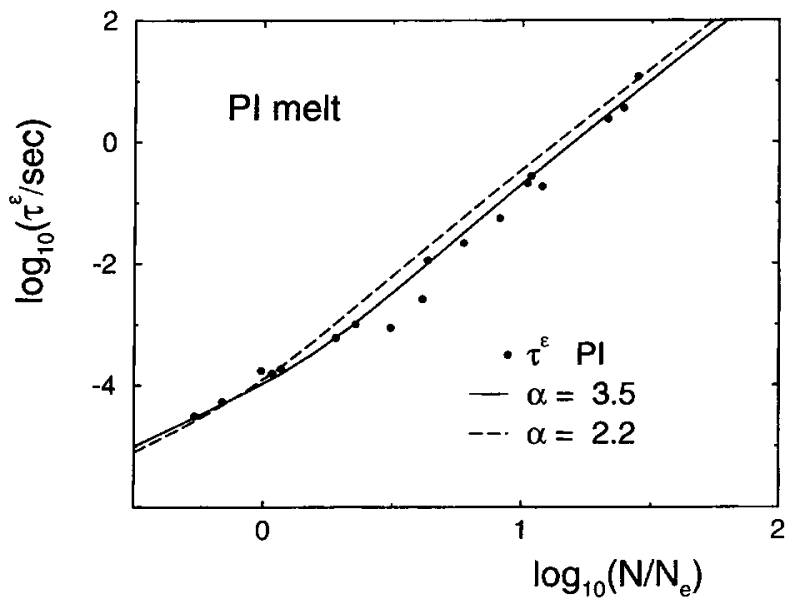

Applications to tracer and self diffusion in other systems (see Tab. 1) have also been carried out. A simultaneous fit to melt self-diffusion data on $\mathrm{PBD}^{89)}, \mathrm{PBDh}^{88)}$ $\mathrm{PI}^{89)}$, polydimethylsiloxane (PDMS ${ }^{90)}$, polyethyleneoxide $(\mathrm{PEO})^{90)}$, and polystyrene (PS) ${ }^{91)}$ is shown in Fig. 11. As theoretically expected, the data do not fall on a single master curve since finite size corrections depend (perhaps weakly in melts) on the nonuniversal structural parameters $\alpha$ and $\delta$. Nevertheless, a good overall fit is obtained with parameters in precisely the range estimated a priori for melts. Small overshooting, or enhancement, of self-diffusion relative to the asymptotic behavior is again found due almost entirely to constraint release corrections. Examples of fits to individual self-diffusion data sets are shown in Fig. 12 and discussed at length elsewhere $^{48)}$.

Application to the tracer diffusion data of Green and $\mathrm{Kramer}^{80)}$ in entangled PS melts is shown in Fig. 13 based on a 2-parameter fitting procedure ${ }^{48)}$. Semi-quantita- 

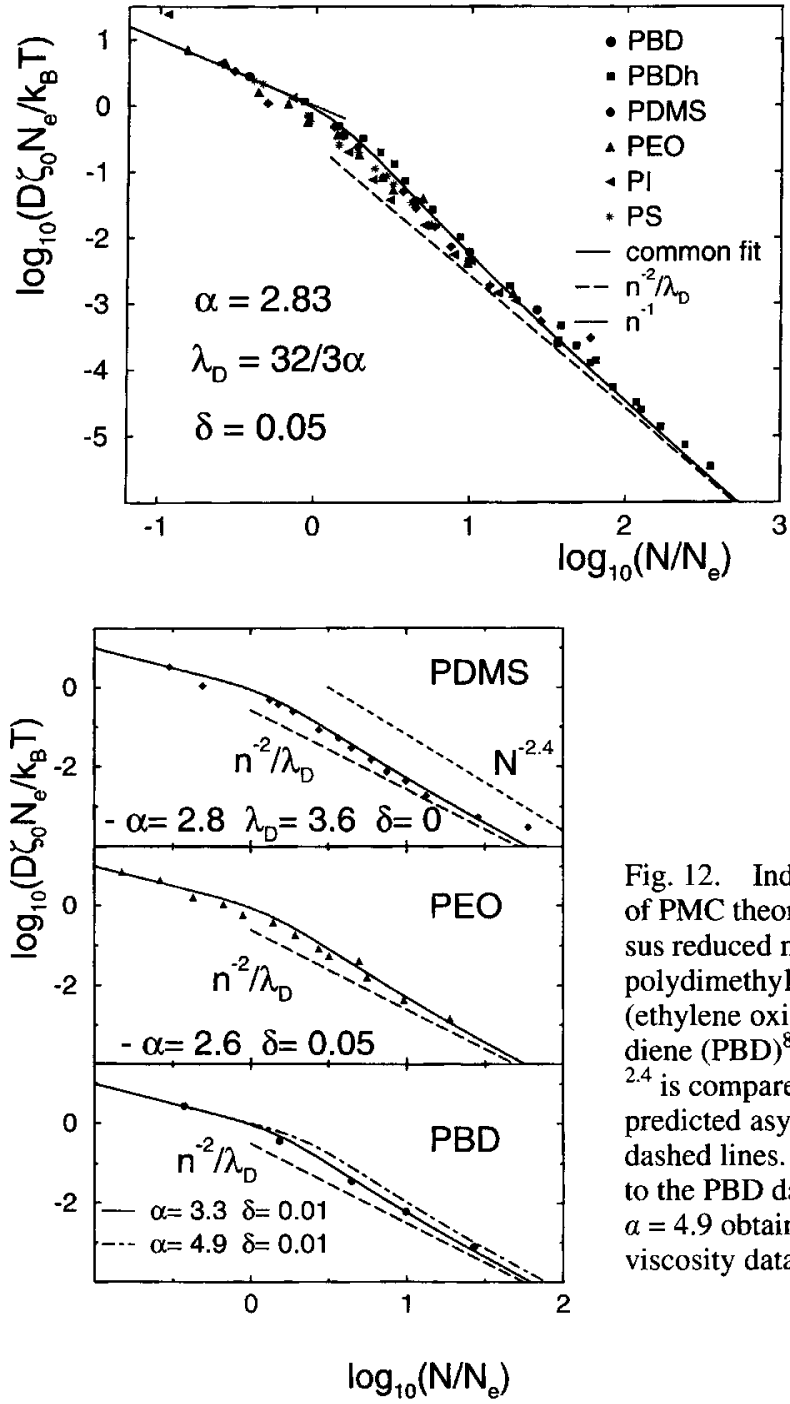
viscosity data
Fig. 11. Self-diffusion constant data for several polymer melts versus molecular weight. A common best fit of all the data is shown, which is not sensitive to the porosity parameter in the range $0<\delta<$ 0.07 . The asymptotes, Rouse and reptationlike, are shown with prefactors corresponding to the fit

Fig. 12. Independent 2-parameter fits of PMC theory to the self-diffusion versus reduced molecular weight data for polydimethylsiloxane (PDMS) ${ }^{90)}$, poly(ethylene oxide) (PEO) ${ }^{90)}$, polybutadiene (PBD) ${ }^{89)}$ melts. A power law of $N^{-}$ 2.4 is compared to the PDMS data. The predicted asymptotes are shown as long dashed lines. The dot-dash curve is a fit to the PBD data using the same value of $a=4.9$ obtained from the best fit to the

tive agreement is obtained, although of somewhat poorer quality than fits based on tube-based models of constraint release for tracer diffusion ${ }^{4,85)}$.

Fig. 14 shows comparisons of theory with self and tracer diffusion constants in PS good solvent solutions. The three concentrations studied by Nemoto et al. ${ }^{81)}$ span the range from semidilute to concentrated. Although the measured shear viscosities obeyed the same $N^{3.5 \pm 0.1}$ law observed in the melt, neither the self nor tracer diffusion constants exhibit $N^{-2}$ behavior even out to $N / N_{\mathrm{e}} \cong 70$. Rather, $D_{\text {self }} \sim N^{-2.7}=0.1$ and $D_{\mathrm{tr}} \sim N^{-2.5 \pm 0.1}$, which is particularly puzzling given the nearly perfect $N^{-2}$ law seen for tracer diffusion in melts. 
Fig. 13. Tracer diffusion coeffients for polystyrene melts as a function of reduced matrix molecular weight and for the indicated different tracer degrees of polymerization. The data ${ }^{80)}$ is shown as solid circles, and the best fit PMC results by solid curves; the relevant parameters are $\alpha=3$ (a priori fixed), $\delta=0.07$, and $\lambda_{D}=1.58$. The corresponding selfdiffusion constants are indicated by the dashed chain curve
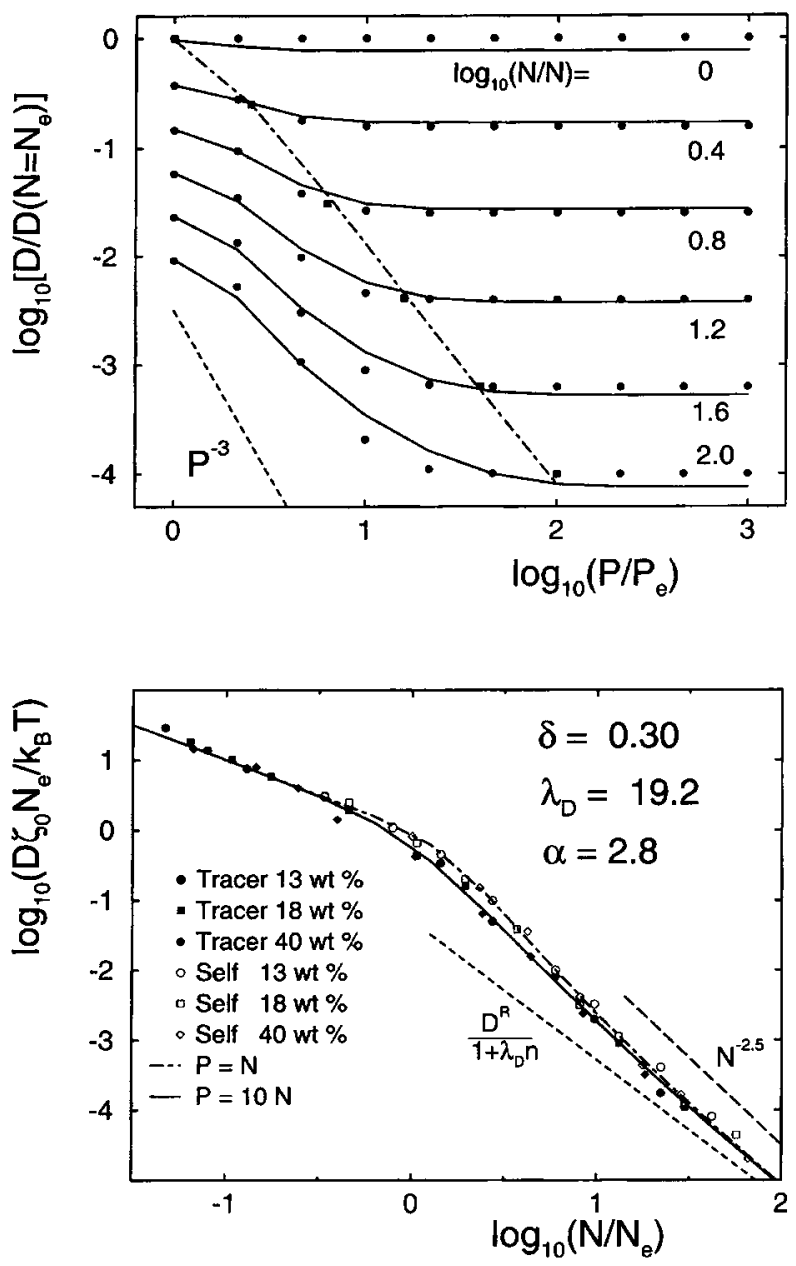

Fig. 14. Self (open symbols; $P=N$ ) and tracer (filled symbols) polystyrene diffusion constants for three concentrations of polystyrene solutions in the good solvent dibutyl phthalate ${ }^{81)}$. The experimentally reported power law $N^{-2.5}$ is indicated by the long dashed line. A common fit ( 2 independent parameters are varied; $a=2.8$ and $P=10 \mathrm{~N}$ are chosen a priori) to the six self (chain curve) and tracer (solid line) data sets for the three concentrations is shown. The predicted asymptotic behavior is shown as the short dashed curve

In the PMC fits of Fig. 14, an independently-determined value of $a=2.8$ was employed based on an analysis of the corresponding viscosity data ${ }^{48)}$. The two parameters $\delta$ and $\lambda_{D}$ were freely varied to simultaneously fit the tracer and self diffusion data at all three solution concentrations. Excellent agreement is found. The rather large (compared to the melt) value of the prefactor $\lambda_{D}$ in Eq. (3.4) required for a good fit is not entirely understood at present, but may reflect limitations in modeling real two component polymer-solvent mixtures with our effective one-component 
description. Independent fits for each concentration have also been carried out, and provide a marginally better description ${ }^{48}$. The individual fit parameters are listed in Tab. 1. For tracer diffusion in the $P \gg N$ regime, constraint release is irrelevant and the anomalous apparent exponent is a consequence solely of constraint porosity corrections. The extracted value of $\delta=0.30$ is in good agreement with estimates based on direct measurements of the density screening length (mesh) and entanglement crossover length in solution, and simulations ${ }^{78)}$ of lightly entangled semidilute solutions. For self-diffusion, constraint release further enhances translational motion, resulting in larger apparent scaling exponents.

In summary, it appears PMC theory with strong finite $N$ corrections provides a unified and consistent description of experimental data for self and tracer diffusion, shear viscosity, and dielectric relaxation time in entangled polymer melts and solutions. To the best of our knowledge, this represents the first theory to achieve this level of understanding.

Predictions for new experiments to more fully test the PMC theory of nonasymptotic dynamical effects have been advanced ${ }^{48)}$. The key idea is to manipulate the three fundamental length scales: polymer density (concentration) screening length $\xi_{\rho}$, entanglement mesh length $r_{\mathrm{c}}$, and tracer radius-of-gyration $R_{\mathrm{g}}$. These nonuniversal structural parameters have a clear physical meaning, are directly measurable, and depend on chemical structure, polymer concentration, temperature, solvent quality, and external pressure. Several specific fruitful areas for more experiments to test novel aspects of the PMC theory can be identified. These include: (i) self and tracer diffusion in good solvents in the unexplored $40-100 \%$ polymer concentration regime, (ii) diffusion measurements in theta solvents where the important length scale ratio $\delta=\xi_{\rho} / r_{\mathrm{c}}$ is larger and more concentration dependent than in good solvents; enhanced porosity corrections and larger effective $N$-scaling exponents are thus predicted, (iii) more transport coefficient studies on different polymer-solvent systems since $\delta$ is chemical structure specific, and (iv) combined self-diffusion and shear viscosity measurements on the same samples in both melts and solutions; such data places severe constraints on our unified theoretical treatment of constraint release and porosity.

\section{Finite frequency effects and non-Markovian relaxation}

Self-consistent constraint release affects the memory functions only for times of order and exceeding their longest relaxation time. Thus, all $t<\tau_{\mathrm{RR}}$ predictions for the time and frequency dependence of observables based on the frozen matrix idealization $(\Phi(t)=1$ in Eqs. (2.34) and (2.35)) should not be affected. However, constraint release is expected to have two important implications for nonzero, but very low, frequencies.

(1) Based on the viscosity predictions described in this section, it is clear self-consistent constraint release always reduces by a non-negligible amount the terminal relaxation time relative to its value under (hypothetical) frozen matrix conditions. A zero frequency consequence is that the asymptotic $\eta \sim N^{3}$ law is experimentally inaccessible in one-component polymer solutions and melts. This prediction implies 
that for experimentally accessible degrees of polymerization there is no real time scale separation between the fluctuating force memory function decay and the physical terminal relaxation time of stress and single chain conformation. Thus, for the relevant values of $\mathrm{N}$ a "non-Markovian" situation applies, where chain motions and fluctuating force time correlations relax on comparable time scales. This lack of time scale separation implies that most of stress relaxation is due to decay of the viscoelastic memory function $M(t)$ via single chain shape fluctuations (internal modes), and not center-of-mass diffusion as would be true in the idealized, but inaccessible, $N \rightarrow \infty$ limit. In addition, one expects the "anomalous" non-reptation $t<$ $\tau_{\mathrm{RR}}$ laws discussed in section IIIB and IIIC, and their frequency domain analogs (which are in good agreement with several experiments), will persist up to the experimentally-observable terminal relaxation time $\tau_{D}$, and thereby control laboratory measurements of stress and dielectric loss moduli, segmental displacements, and incoherent dynamic structure factors. The asymptotic analytic predictions in section III for the last (third) intermediate time regime, $\tau_{\mathrm{RR}} \ll t \ll \tau_{D}$, are thus not expected to be observable for chains of experimentally relevant length.

(2) Under all practical situations, the long time tails of the memory functions, which emerge only for $t>\tau_{\mathrm{RR}}$ under frozen matrix conditions due to the presumed diffusive decay of the fluctuating forces on $k R_{\mathrm{g}}<1$ scales, will be "cut off" by the constraint release process. This feature can have significant consequences for frequency domain measurements in the terminal regime of $\omega \tau_{D}<1$. For example, in the absence of self-consistent constraint release the Laplace transform of the viscoelastic memory function in the low frequency regime is of the form

$$
M(z) \cong M(z=0)\left[1-a\left(\frac{N_{\mathrm{e}}}{N}\right)^{1 / 4} \sqrt{z \tau_{D}}\right], \quad z \tau_{D} \ll 1
$$

The zero frequency Markovian limit is well-behaved (the tail is integrable), but a nonanalytic correction in the frequency domain does appear. Although of negligible consequence in the formal $N \rightarrow \infty$ limit, the very weak $N^{-1 / 4}$ damping of its amplitude implies significant consequences for experimentally relevant values of $N / N_{\mathrm{e}}$ are likely. This conclusion is verified in numerical studies discussed in section VI. It is found that $\omega \tau_{D}<1$ stress moduli are of excessive breadth, in disagreement with experiment. However, constraint release effectively removes the long time tail, and hence its incorrect low frequency consequences.

\section{Tracer diffusion in gels and networks}

The diffusion of tracer polymers through amorphous media is of great interest in physical and biological science and technology ${ }^{83)}$. The original reptation/tube theory was formulated for motion of a chain in a crosslinked network ${ }^{2,3)}$. Gel electrophoresis is a powerful, but still not well understood, method to characterize biopolymers $^{92)}$. The unique aspect of PMC theory is that it provides connections between tracer dynamics and the diversity of possible gel structures, gel-solvent-tracer interactions, and tracer conformation. The constraint release mechanism is inoperative, so the prime physical issue is the role of constraint porosity. 


\section{A. General formulation and asymptotic predictions}

Gels generally possess a fractal structure characterized by a mass scaling exponent $D_{\mathrm{F}}$, and a pore or mesh size $\xi_{\mathrm{g}}$. Gel formation by crosslinking of polymer solutions is expected to yield fractal exponents in the range $D_{\mathrm{F}}=5 / 3-2$. Simple models for the gel density correlations are

$$
S(k)=\frac{S_{0}}{\left(1+k^{2} \xi_{\mathrm{g}}^{2}\right)^{D_{\mathrm{F}} / 2}}, \quad S(k)=\frac{S_{0}}{1+k^{2} \xi_{\mathrm{g}}^{2}}+S_{\mathrm{xc}} \mathrm{e}^{-\left(k \xi_{\mathrm{xc}}\right)^{0.7}}
$$

The second form has been shown to fit data quite well ${ }^{93)}$, and accounts for both annealed gel density fluctuations and quenched inhomogenities associated with crosslinking. The characteristic length scale of the quenched disorder, $\xi_{\mathrm{xc}}$, is generally much larger than $\xi_{\mathrm{g}}$. In the effective medium PMC spirit, quenched and annealed disorder are treated in the memory functions solely via $S(k)$. In contrast to "hard" silica-like gels"94), "soft" gels formed by lightly crosslinking polymer solutions will display a quenched in elastic mesh length, $r_{c}$, in analogy with their entangled solution precursors. Typical values of the constraint porosity parameter, $\delta=\xi_{\mathrm{g}} / r_{\mathrm{c}}$, are expected to be comparable to solution values, unless a "strangulation regime" applies due to a very high degree of crosslinking ${ }^{95)}$.

The nature of tracer conformation in a fractal gel medium is a complex, nonuniversal question which remains poorly understood theoretically and experimentally ${ }^{83,93)}$. The polymer mass scaling exponent defined by $R_{\mathrm{g}} \sim N^{v}=N^{1 / d_{\mathrm{F}}}$, may depend on degree of polymerization range, gel density, degree of crosslinking, etcetera. As a simple model, the following form is adopted for the tracer structure factor

$$
\omega(k)=N\left(1+c_{\nu} k^{2} R_{\mathrm{g}}^{2}\right)^{-d_{\mathrm{F}} / 2}
$$

where $d_{\mathrm{F}}$ is the tracer fractal dimension, and $c_{\nu}$ is chosen to reproduce the exact large $k$ self-similiar power law behavior ${ }^{48)}$.

Based on the above simple structure factor models, the generalization of Eq. (4.3) to the tracer-gel problem in the frozen matrix limit yields

$$
D^{\mathrm{tr}}=\frac{k_{\mathrm{B}} T}{\zeta_{\mathrm{0}} N}\left\{1+\lambda_{\mathrm{g}} N^{4-\left(2 d / d_{\mathrm{F}}\right)} K_{\mathrm{g}}\left(R_{\mathrm{g}} / \xi_{\mathrm{g}}, \xi_{\mathrm{g}} / r_{\mathrm{c}}\right)\right\}^{-1}
$$

where $d$ is the space dimension and the correction function $K_{\mathrm{g}}$ is precisely defined elsewhere $^{48)}$. Simple Rouse dynamics (no hydrodynamic interactions) has been adopted for the "bare" unentangled diffusion. The parameter $\lambda_{\mathrm{g}}$ is proportional to the mean square force exerted by the gel (mediated by the solvent) on a tracer segment in analogy with Eq. (3.4). This strength parameter is not easily predicted. We expect it to be highly variable since the typical system is a ternary gel-solvent-tracer mixture, and specific attractive or repulsive effective interactions between the tracer and gel strands are possible.

In the asymptotic large tracer limit $\left(R_{\mathrm{g}} \gg r_{\mathrm{c}}, \xi_{\mathrm{g}}\right)$, the correction function $K_{\mathrm{g}} \rightarrow 1$, and $D^{\mathrm{tr}} \sim N^{-\kappa}$ with $\kappa=5-\left(2 / d_{\mathrm{F}}\right) d$. This asymptotic result is independent of gel frac- 
tal dimension since for such a large tracer the gel appears statistically homogeneous. Examples of possible asymptotic behaviors are: $D \sim N^{-2}$ in 3-dimensions for ideal coil tracers, and, curiously, $D \sim N^{-2}$ in 2-dimensions for swollen tracer chains where $d_{\mathrm{F}}=4 / 3$. The latter prediction is in excellent agreement with 2 -d computer simulations by Slater and Wu of self-avoiding walk (SAW) chains in a dense obstacle net ${ }^{96)}$. Interestingly, the $N^{-2}$ "reptation law" occurs by accident for the $d=2$ case, and does not agree with the proper reptation prediction for swollen coils in $d=2$ : $D_{\text {rep }} \sim R_{\mathrm{g}}^{2} / N^{3} \sim N^{-3 / 2}$.

\section{B. Model calculations and experimental comparisons}

In the most common experimental situation, the correction function $K_{\mathrm{g}}$ in Eq. (5.3) is not unity. It quantifies the constraint porosity effects associated with finite gel pore size and dynamic entanglement length. Apparent exponents which are larger than the asymptotic values are expected, in analogy with the entangled solution problem. In contrast to phenomenological "entropic barrier" ${ }^{82,83)}$ and "enthalpic trap" ${ }^{97)}$ approaches, Eq. (5.3) describes not only an intermediate $N$ anomalous transport regime, but also the low and high $N$ limiting cases and crossovers.

Fig. 15 shows model calculations of $D^{\mathrm{tr}}$ based on Eq. (5.3) for specific choices of tracer and gel fractal dimension, interaction strength parameter $\lambda_{\mathrm{g}}$, and a fixed $\delta$. Gel mesh length is denoted by reporting the degree of polymerization of a tracer with a radius-of-gyration such that $\xi_{\mathrm{g}}=R_{\mathrm{g}}(N)$. There are several noteworthy trends. (i) Deviations from the bare Rouse behavior emerge for $R_{\mathrm{g}} / \xi_{\mathrm{g}}<1$, but asymptotic behavior $\left(K_{\mathrm{g}} \rightarrow 1\right)$ does not appear up to very high tracer degrees of polymerization. (ii) Apparent intermediate power laws appear with varying exponents, $D^{\text {tr }} \sim N^{-y}$ with $y=2.3-2.8$, depending on system-specific parameters. This is a particularly dramatic effect since the true asymptotic molecular weight dependence for the tracer fractal dimension employed in Fig. 15 is $D \sim N^{-1.4}$, which is even weaker than reptation-like scaling. (iii) The steepest molecular weight dependences fall in a range where the tracer $R_{\mathrm{g}}$ significantly exceeds the gel pore size $\xi_{\mathrm{g}}$. This finding appears to disagree with entropic barrier ideas ${ }^{82,83)}$, where maximal non-asymptotic behavior is centered around $R_{\mathrm{g}} \cong \xi_{\mathrm{g}}$. (iv) An upper bound to the effective exponents can be deduced from the small tracer size limit of Eq. (5.3). For $R_{\mathrm{g}} / \xi_{\mathrm{g}} \ll 1$, it follows that for large asymptotic prefactors $\lambda_{\mathrm{g}}$, and/or large pore sizes, an upper bound is obtained as $D^{\text {tr }} \sim N^{-\theta}, \theta=5-\left(2 / d_{\mathrm{F}}\right)\left(d-D_{\mathrm{F}}\right)$. For the case of Fig. 15 this estimate is $D^{\text {tr }} \sim N^{-3.4}$. (v) Anomalous, non-asymptotic exponents are not directly associated with the dominance of alternative (postulated) real space motional mechanisms, but rather with modifications of the spatially resolved entanglement constraints due to tracer-gel interactions and structure.

Applications to several data sets ${ }^{95,98)}$ have been recently carried out ${ }^{48)}$. Here, we only discuss measurements by Hoagland and coworkers ${ }^{98)}$ of the low field gel electrophoresis mobility, $\mu=N D^{\mathrm{tr}}$. Polystyrenesulfonate (PSS) diffusing in crosslinked polyacrylamide (PAA) entangled solutions was studied at three gel concentrations $c$ (gel weight percent). The data is shown in Fig. 16, along with fits of PMC theory. The calculations employed the swollen coil exponents for tracer and gel, $d_{\mathrm{F}}=D_{\mathrm{F}}=$ 

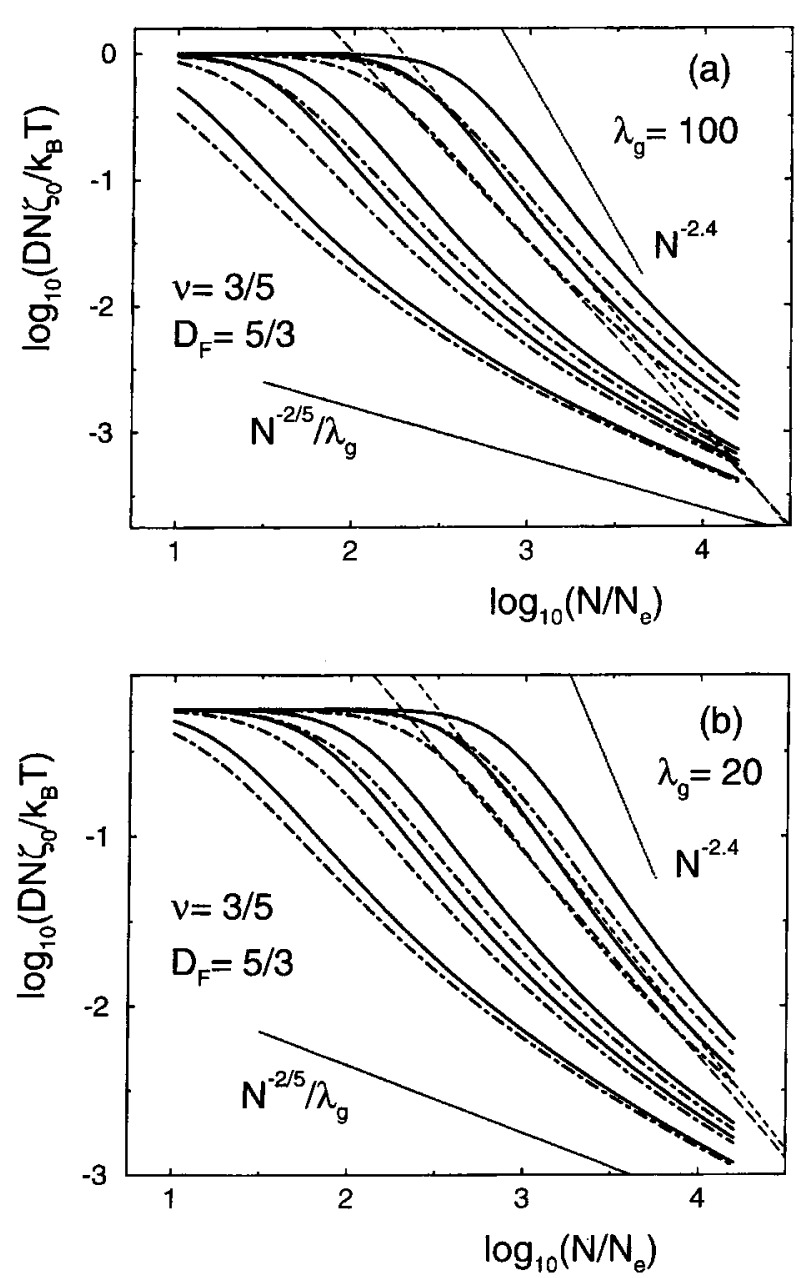

Fig. 15. Tracer diffusion constants (normalized by the Rouse value) versus tracer degree of polymerization in gels with various pore sizes $\xi_{\mathrm{g}}$ chosen to equal the tracer $R_{\mathrm{g}}$ for $N / N_{\mathrm{e}}=10,50,100,500$, 1000 ; the curves are monotonically lower with increasing $N / N_{\mathrm{e}}$. Good solvent conditions are chosen, and the tracer-gel interaction strength parameter is $\lambda_{\mathrm{g}}$ $=100$ and 20 in frames (a) and (b), respectively. The thick solid lines are for the "soft polymer gel" case discussed in the text with a fixed, dense solution-like value of $\delta=\xi_{\rho} / r_{\mathrm{c}}=0.3$. The chain curves correspond to a "hard gel"model as discussed elsewhere $^{48)}$. The thin line labeled $N^{-2 / 5} / \lambda_{\mathrm{g}}$ is the asymptotic behavior which applies only beyond $N / N_{\mathrm{e}} \cong 10^{8}$. The maximal intermediate slope is shown as the thin dotted line

$5 / 3$, and $\delta$ was a priori fixed at a solution-like value of 0.3 . Least square fits of the PMC theory Eq. (5.3) to each gel concentration data set were carried out based on variable gel-tracer interaction strength parameter $\lambda_{\mathrm{g}}$ and gel pore size $\xi_{\mathrm{g}}$. The near constancy of the extracted gel-tracer interaction parameter seems physically sensible. Gel pore sizes are reported in Fig. 16 by stating the PSS degree of polymerization $N$ giving $R_{\mathrm{g}, \mathrm{PSS}}(N)=\xi_{\mathrm{g}}$. The physically expected decrease of gel pore size with increasing gel density is observed, and is in reasonable agreement ${ }^{48)}$ with the dependence expected for semidilute good polymer solutions ${ }^{2)}$.

Given the uncertainty about structural parameters in the complex polymer-solvent-gel environment, the agreement of theory with the data in Fig. 16 is quite satisfying. We again note that the steepest region of the mobility versus molecular weight curve lies in a region where $R_{\mathrm{g}} / \xi_{\mathrm{g}} \gg 1$, contrary to expectations based on the entropic barrier model ${ }^{82,83)}$. The effective $N$ scaling exponent of the mobility is much lar- 


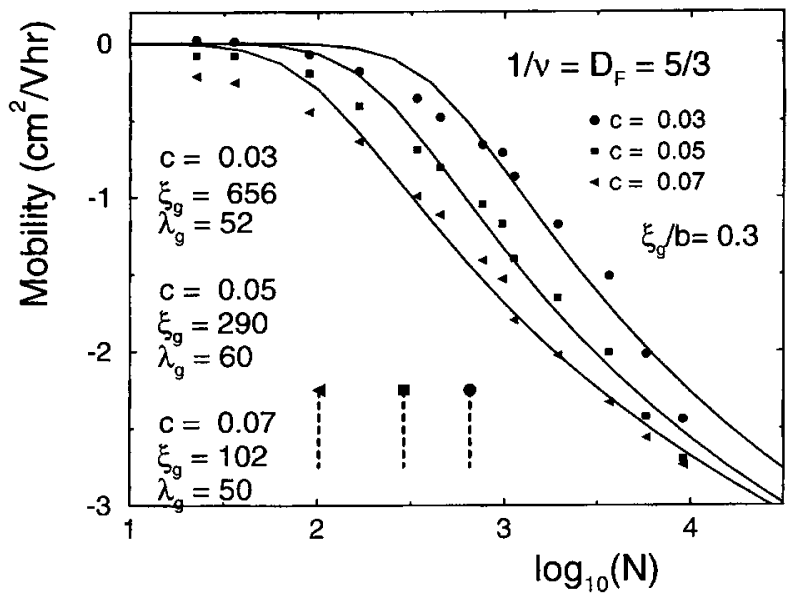

Fig. 16. Low field mobilities of polystyrenesulfonate (PSS) tracers in three polyacrylamide (PAA) gels ${ }^{98)}$ of different concentrations $c$ (gel weight percent) as a function of degree of polymerization. The full lines are the PMC fits based on the soft gel model under good solvent conditions with the parameters indicated. Gel pore sizes, $\xi_{\mathrm{g}}$, are expressed by stating the degree of polymerization, $N$, of a PSS tracer with identical radius-of-gyration. The vertical lines denote the tracer degrees of polymerization where $R_{\mathrm{g}}(N)=\xi_{\mathrm{g}}(c)$. The parameter $\delta=\xi_{\rho} / r_{\mathrm{c}}=\xi_{\rho} / b=0.3$ was fixed a priori based on prior solution studies

ger than the reptation value of -1 . Future theory-experiment comparisons for tracer diffusion would be greatly facilitated by the availability of more experimental information concerning the gel structure factor, tracer conformations, and polymer-gel interactions. Controlled manipulation of physiochemical properties to rationally vary the key structural parameters $\delta, a$, and $\lambda_{D}$ could also allow more penetrating tests of the PMC theory of strong finite size corrections and structure-dynamics connections in gels.

\section{Numerical polymer mode coupling theory}

Mode-coupling theories are not valid at very short times or distances since they are constructed to treat collective dynamical processess which require a finite time to emerge from many microscopic collisions ${ }^{50-54)}$. In prior sections a simple analytic time domain approach to treat the bare/mode-coupled crossover was adopted: Rouse dynamics for $t<\tau_{\mathrm{c}}$ and mode-coupled dynamics for $t>\tau_{\mathrm{c}}$. The crossover time was estimated using the RR theory. This simple model is not adequate for truly quantitative numerical studies. The purpose of the latter is to more precisely quantify prefactors and finite $N$ corrections, and predict (not assume) the nature of the crossover from Rouse to entangled dynamics. Practical questions relevant to experimental (and simulation) systems include the breadth of the crossover connecting different 
(asymptotically distinct) time and frequency regimes, and apparent time, frequency, and $N$ scaling exponents.

Numerical work performed to date ${ }^{40,41,77)}$ has been based on the original version of PMC theory ${ }^{36)}$ : a literal (naive) frozen matrix approximation with collective density fluctuations as a slow variable, i.e. $S(k, t) \cong S(k)=S(0) /\left[1+\left(k \xi_{\rho}\right)^{2}\right]$. Thus, selfconsistent constraint release and dynamic mesh porosity corrections ${ }^{48)}$ are ignored. Numerical results based on this level of PMC theory should be useful for studying the Rouse/mode-coupling crossover, the influence of probe shape fluctuations in the pre-terminal relaxation regime, finite size modification of anomalous diffusion time scaling exponents, and smearing of well-defined intermediate time regimes due to broad crossover effects. However, finite size deviations from the $N^{3}$ law, or nonasymptotic self and tracer diffusion scaling exponents, are not expected to be properly captured. These expectations are borne out in the numerical results discussed below and elsewhere ${ }^{77)}$.

\section{A. Numerical crossover model}

The general problem of describing the crossover between unentangled Rouse and entangled dynamics is a very difficult one. Indeed, its analog for caging dynamics in simple liquids remains largely unsolved ${ }^{50-52)}$. Oversimplified crossover schemes must be invoked based on physical sensibility and mathematical convenience. Szamel and Schweizer have considered two numerical crossover models ${ }^{40,41,77)}$. The first scheme simply turns off the PMC memory functions at short times $t<\tau_{\mathrm{c}}$ via a "switching function", thereby enforcing Rouse dynamics over the appropriate short time and distance scales. This procedure is sensible in the time domain, but yields negative values of loss moduli in the frequency domain. We do not believe this implies any fundamental difficulty, rather only that frequency domain response functions are very sensitive to the crossover model.

The second method investigated was to introduce a crossover in normal mode space. The memory function is required to be Rouse-like for short wavelength normal modes (high $p$ index), thereby indirectly, and more approximately, introducing a crossover in the time/real space domain. A similiar procedure has been employed by des Cloizeaux ${ }^{72)}$ within the reptation/tube framework. The precise implementation introduces a switching function, $\chi(p)$, at the fundamental level of the PMC memory functions. This function varies from unity to zero as the normal mode index $p$ increases, i.e., $\Gamma_{p}(t) \rightarrow \chi(p) \Gamma_{p}(t)$ in Eq. (2.31), and applies for the calculation of all dynamical observables.

Two specific, minimum parameter switching functions have been studied corresponding to exponential and step function crossovers ${ }^{40,41)}$

$$
\chi_{1}(p)=\lambda_{1} \exp \left(-p N_{\mathrm{c}}^{(1)} / N\right), \quad \chi_{2}(p)=\lambda_{2} H_{-}\left[p-N / N_{\mathrm{c}}^{(2)}\right]
$$

where function $H_{-}(x)=1(0)$ for $x<0(x>0)$. The empirical parameter $\lambda_{j}$ is a pure number which adjusts the absolute strength of the mode-coupling memory functions. The parameter $N_{\mathrm{c}}$ determines (roughly) the spatial scale, $r_{\mathrm{c}} \cong \sigma N_{\mathrm{c}}{ }^{1 / 2}$, beyond which 
mode-coupled dynamics applies. The absolute magnitude of $N_{\mathrm{c}}$ is not a priori calculable. However, based on the RR crossover theory of section IIB, and the PRISM predictions for Gaussian string polymer fluids, we estimate its solution density $(\rho)$ dependence a priori using the results discussed above Eq. (2.18):

$$
N_{\mathrm{c}}(\rho)=N_{\mathrm{c}, \text { melt }}\left(\rho_{\mathrm{m}} \sigma_{\mathrm{m}}^{3} /\left(\rho \sigma^{3}\right)\right)^{2}
$$

Here, $\rho_{\mathrm{m}}\left(\sigma_{\mathrm{m}}\right)$ is the melt density (segment length), and $N_{\mathrm{c} \text {,melt }}$ is a system-specific degree of polymerization in the melt state the chemical structure dependence of which can also be estimated based on the RR crossover theory ${ }^{42)}$ of section IIB. The parameters $\lambda$ and $N_{\mathrm{c} \text {,melt }}$ are determined by requiring the PMC theory predictions for the crossover degrees of polymerization for the melt self-diffusion coefficient $\left(N_{D}\right)$ and shear viscosity $\left(N_{\eta}\right)$ take on specified values, as suggested by experiment or simulation. The self-diffusion crossover degree of polymerization is defined by the condition $D / D_{\mathrm{R}}=1 / 2$. The crossover $N_{\eta}$ is defined from the intersection of the extrapolated small (Rouse) and high (entangled) $N$ theoretical predictions for the shear viscosity. The density dependence of all properties are predictable based on Eq. (6.2) and the use of PRISM theory (or other sources) for the equilibrium information.

For the specific numerical results discussed below, Szamel and Schweizer have employed string PRISM theory ${ }^{42,60)}$, a reduced melt density of $\rho_{\mathrm{m}} \sigma^{3}=0.5$ (corresponding to a melt screening length $\xi_{\rho} \cong 2 \sigma$ ), a Lorentzian single polymer structure factor $\omega(k)$, the Akcasu form of Eq. (2.33), and an upper wavevector cuttoff of $\sigma^{-1}$ in the PMC memory functions. Theta solutions of $20 \%$ and $60 \%$ polymer volume fraction are modeled using values of $\rho_{\mathrm{m}} \sigma^{3}=0.1$ and 0.3 , respectively. For simplicity, the diffusion constant and viscosity crossover degrees of polymerization are set equal: $N_{D}=N_{\eta}=70$, which is a reasonable segmental value based on melt experiments ${ }^{1,65)}$ or simulations ${ }^{31)}$. Results are shown using crossover model 1 , although preliminary studies using model 2 yield predictions which are not very sensitive to the switching function. The numerical details of performing the required normal mode sums, integrals, and Fourier and Laplace transforms are described elsewhere $^{77)}$.

\section{B. Model calculations and experimental comparisons}

The fundamental quantities are the memory functions. Representative results in the time domain are shown in Figs. 17a and 17b. Although the various analytically predicted asymptotic regimes are somewhat visible for larger $N$, they are considerably smeared by rather broad crossovers. Moreover, the approximate power law regimes are characterized by $N$-dependent effective exponents. Note that significant decay of the viscoelastic memory function occurs at "intermediate" times $t \ll \tau_{\mathrm{RR}}$ in the von Schweidler manner of Eq. (2.38). The finite size mechanism is dynamical polymer shape fluctuations which are effective for $k R_{\mathrm{g}}>1$ scale contributions to $M(t)$. This initial, self-similiar decay occurs well before the long time $t^{-3 / 2}$ tail emerges corresponding to the final decay of viscoelastic entanglement forces via 
probe center-of-mass diffusion. For example, for the $N / N_{\mathrm{c}} \cong 140$ case, $M(t)$ decays by nearly a factor of 100 before the long time tail regime becomes dominant. Although such intermediate time decay via internal mode fluctuations is not effective in relaxing $M(t)$ in the $N \rightarrow \infty$ limit, for experimentally accessible $N$ it is very important since $M(t)$ can be reduced to nearly insignificant magnitude via the shape fluctuation mechanism.
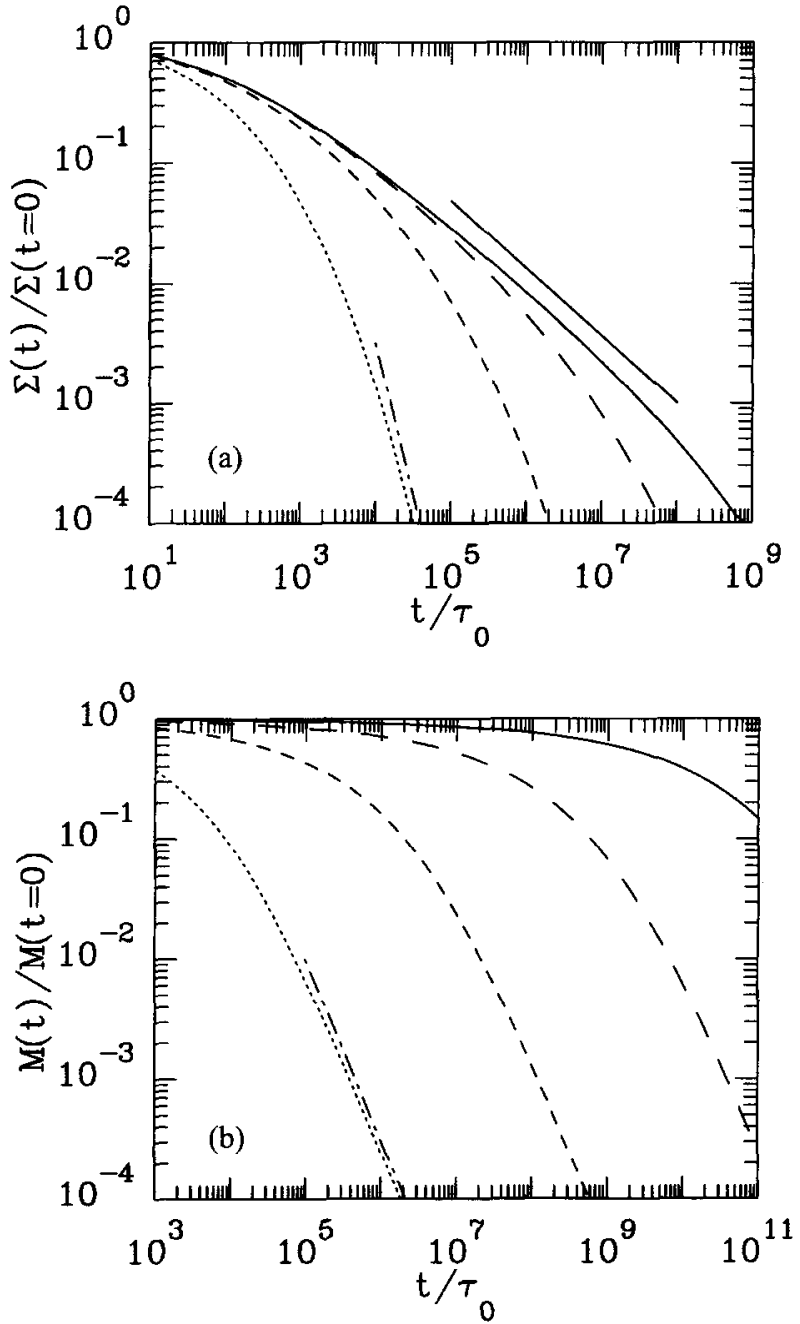

Fig. 17. Logarithmic plots of the normalized (a) diffusional and (b) viscoelastic memory functions as a function of reduced time, where here $\tau_{0}=\beta \sigma^{2} \zeta_{0}$ is the elementary Rouse model time scale. Numerical results are for melt-like conditions and are shown for $N=$ $10^{2}$ (short dashed), $10^{3}$ (intermediate dashed), $10^{4}$ (long dashed), and $10^{5}$ (solid curve). A dash-dot line of slope $-3 / 2$ is shown in (b), and a dash-dot (solid) line of slope $-7 / 2$ $(-9 / 16)$ is indicated in (a)

Results for the Rouse-normalized self-diffusion coefficient and shear viscosity are shown in Figs. 18 and 19, respectively. For self-diffusion, the asymptotic $N^{-2}$ scaling is recovered at very large $N$, but there is a significant "overshooting" regime where effective power laws of $N^{-\theta}, \theta \cong 2.2 \pm 0.1$, emerge at intermediate molecular 
Fig. 18. Self-diffusion constant normalized by its Rouse value as a function of degree of polymerization for $N_{D}=$ 70 and the melt-like density (solid curve), $60 \%$ solution (long dashed), and $20 \%$ solution (short dashed) cases. A dash-dot line of slope -1 is also shown

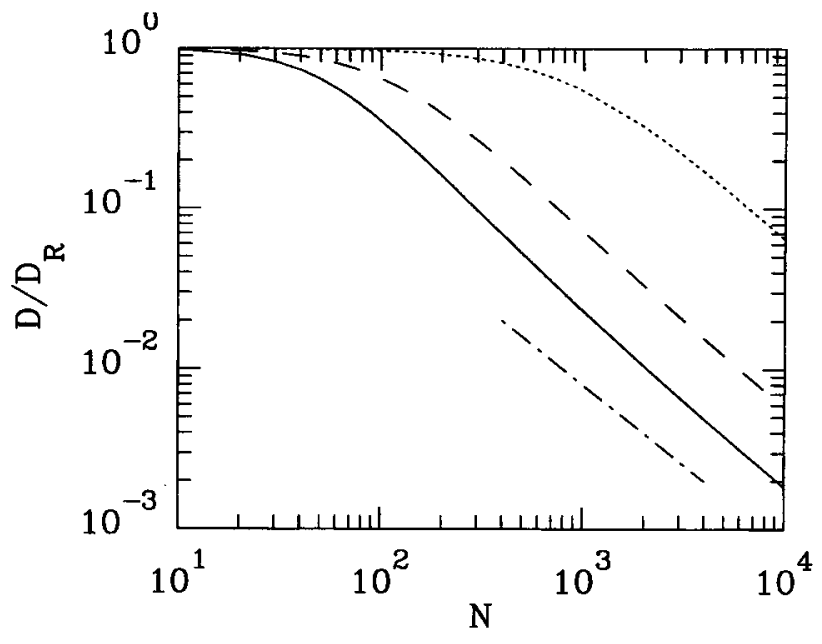

Fig. 19. Shear viscosity divided by the reduced density in arbitrary units as a function of degree of polymerization for $N_{\eta}=70$ and melt-like (solid curve), $60 \%$ solution (long dashed), and $20 \%$ solution (dash-dot) cases. The short dash curve represents the extrapolated Rouse behavior. A solid line of slope 3.5 is also shown

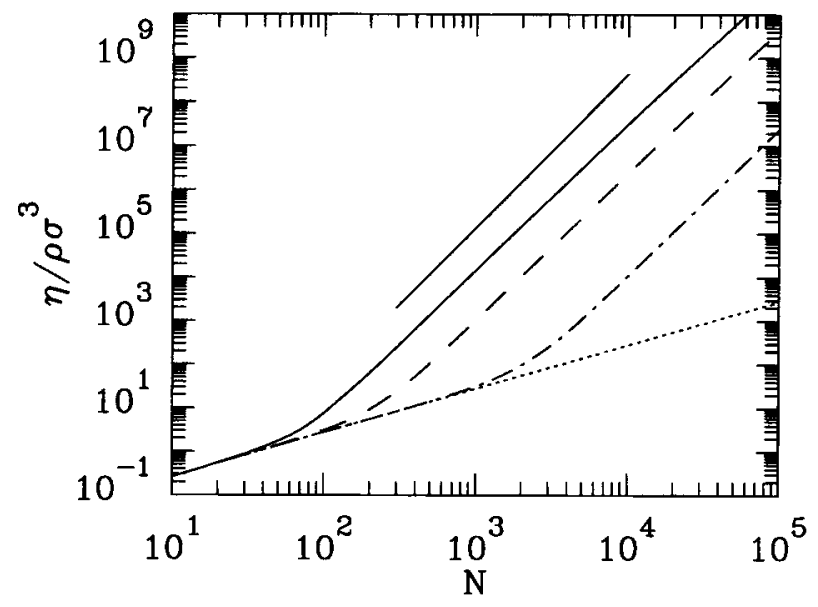

weights. Moreover, $\theta$ tends to weakly decrease upon dilution of the melt, contrary to experimental behavior. As expected, the deviations from asymptotic behavior are significantly less than the predictions for solution tracer diffusion obtained in section IV which accounted for entanglement mesh porosity and constraint release.

Out to $N / N_{\eta} \cong 300$, the viscosity follows a $N^{3.5}$ scaling law nearly independent of polymer density. Although in apparent accord with experiment, this agreement appears to be for incorrect physical reasons. The terminal relaxation time, defined from the maximum of $G^{\prime \prime}(\omega)$ as $\tau_{D}=2 \pi / \omega_{\max }$, is found to scale very nearly as $N^{3}$. This is expected based on the results of section IV since self-consistent constraint release is not taken into account in the numerical studies. Thus, the origin of the $N^{3.5}$ law for the viscosity in Fig. 19 is a spurious dependence of the overall amplitude of $G^{\prime}(\omega)$ and $G^{\prime \prime}(\omega)$, and hence the plateau modulus, on degree of polymeriza- 
tion. Over the experimentally relevant range of $N$ studied in Fig. 19, it is found that $G_{N} \sim N^{0.4}$ (and hence $J_{\mathrm{e}} \sim N^{-0.4}$ for the compliance) for $N / N_{\eta}=20-100$, although in the asymptotic $N \rightarrow \infty$ limit the plateau modulus and creep compliance do approach limiting, $N$-independent values ${ }^{77}$ ). As demonstrated below, this defect of the crossover model affects the storage and loss moduli in an identical manner, and only as an overall scale factor. Thus, predictions for the normalized moduli, their frequency dependence as a function of $N$, and their polymer density dependence at fixed $N$, are not perturbed by this defect of the numerical crossover model. The polymer density dependence of both $N_{D}$ and $N_{\eta}$, and the large $N$ values of $D$ and $\eta$, are all found to be in close agreement with the analytic scaling laws discussed in section IIIA (see Eqs. (3.5)).

Center-of-mass and (chain-averaged) segmental mean square displacements are shown in Fig. 20. Broad crossovers between the analytically predicted regimes are
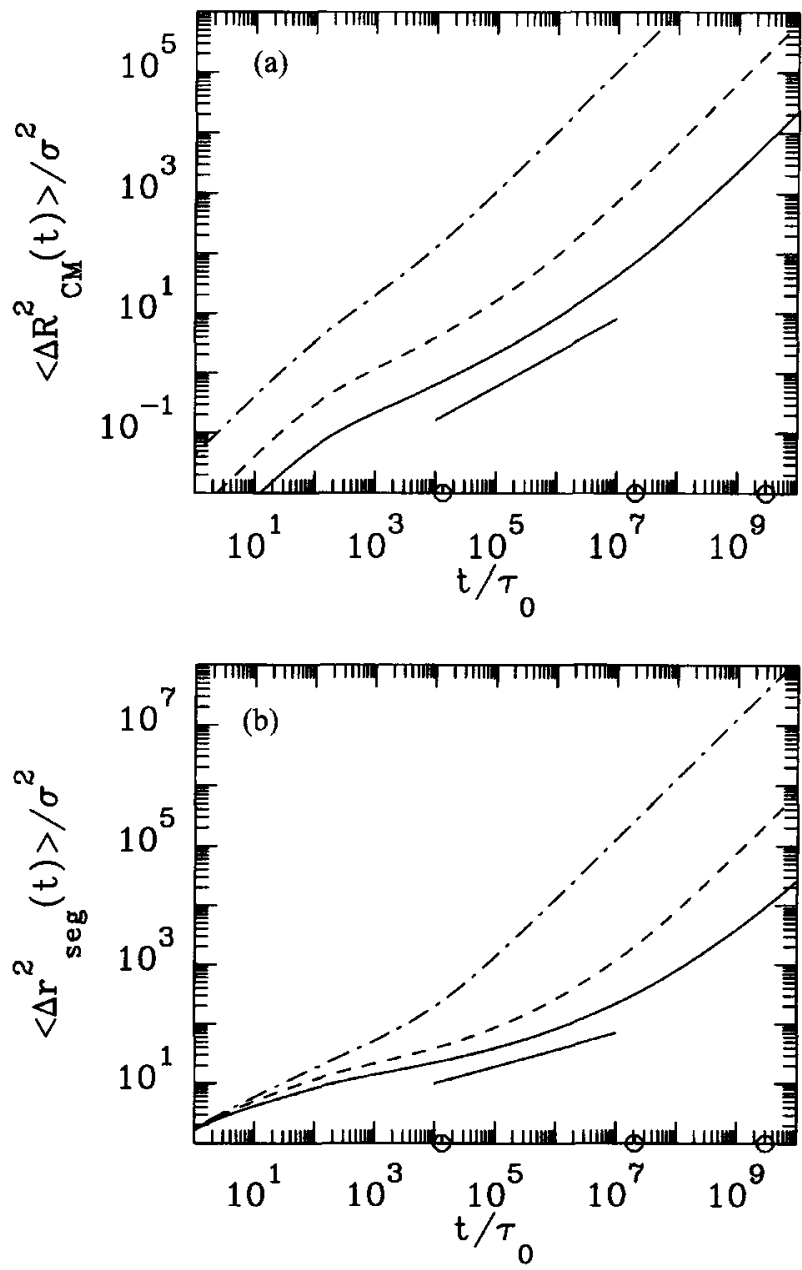

Fig. 20. Dimensionless center-of-mass (a) and chain-averaged segmental (b) displacements as a function of reduced time for three reduced values of chain length $N / N_{D}=2$ (dashdot), 20 (dashed), and 100 (solid) and the melt-like density. The circles on the lower time axis denote the times at which a mean square displacement of $R_{\mathrm{g}}^{2}$ occurs. A solid line of slope $9 / 16$ and $9 / 32$ is shown in part (a) and (b), respectively 
evident, and the large $N$ scaling exponents are approached from above with increasing entanglement. The finite size smearing effects seem more noticable for the center-of-mass dynamics. Enhanced diffusion of end segments relative to central parts of the chain is predicted by PMC theory (and also Rouse dynamics), but is a much smaller effect than for reptation/tube dynamics $3,34,77$ ).

Fig. 21 presents the segmental displacement results in an apparent time-dependent diffusion constant format following Eq. (3.16). As discussed in section IIID, this plot is relevant to recent PFG-NMR measurements ${ }^{74,75)}$. For the experimentally relevant cases $\left(N / N_{\mathrm{e}}<100\right)$, power law behavior over one or two orders of magnitude in time occurs, although the effective exponent depends on the specific time regime examined and degree of polymerization. Expressed in the form adopted by the experimental studies, $D_{\text {app }}(t) \sim t^{-\Delta}$, the effective exponent falls in the range $\Delta \cong 0.6$ \pm 0.1 . The latter values appear to be in agreement with experiments ${ }^{74)}$, which often seem to be larger than the pure reptation prediction of $A=0.5$. Moreover, PMC theory predicts systematic, although rather weak, trends: $\Delta$ tends to increase as shorter time regimes (displacements) are analyzed for fixed $N / N_{D} \gg 1$, or if larger $N / N_{D}$ melts are examined at fixed $t / \tau_{D}$. For the lightly entangled $N / N_{D}=2$ case the behavior is essentially Rouse-like with $\Delta=1 / 2$. New experiments on a single chemical system which systematically varies $N / N_{D}$ and the range of $t / \tau_{D}$ probed would be valuable to more quantitatively test the numerical PMC theory predictions for apparent time scaling exponents, and also the fundamental question of whether the incoherent scattering function depends on external wavevector in a Gaussian or power law fashion.

Numerical predictions for the stress relaxation loss modulus of melts at various degree of entanglement are shown in Fig. 22. A normalized plotting format is employed to emphasize the $N$-dependence of the shape of $G^{\prime \prime}(\omega)$. The high frequency side of the loss maximum $G^{\prime \prime}(\omega)$ follows an apparent power law with an

Fig. 21. Dimensionless, apparent timedependent diffusion constant as a function of reduced time under melt-like conditions and for $N / N_{D}=2$ (short dashed), 10 (dashed), 20 (long dashed) and 100 (solid). Here, $D_{0}=$ $\left(\beta \zeta_{0}\right)^{-1}$ is a free segment diffusion constant and $\tau_{\mathrm{d}}=R_{\mathrm{g}}^{2} / D$ is a characteristic diffusion relaxation time

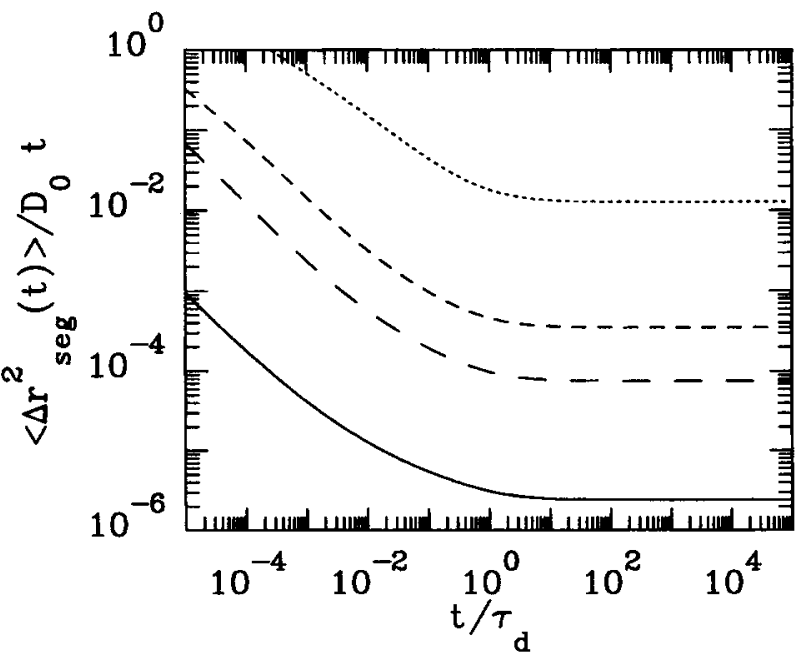


exponent which decreases rather remarkably weakly with $N$. Moreover, even for the most strongly entangled case $\left(N / N_{\eta}=300\right)$ the apparent scaling exponent is small, roughly $G^{\prime \prime}(\omega) \sim \omega^{-0.25}$, which is very far from the pure reptation law of $\omega^{-0.5}$. These PMC predictions are in excellent agreement with recent experimental studies of polybutadiene and polystyrene melts by Winter and coworkers ${ }^{22)}$, and the asymptotic PMC analytic results of section III. Thus, the numerical studies again emphasize that dynamic polymer shape fluctuations are very effective at relaxing entanglement constraints, i.e., $M(t)$, for the finite $N$ systems of experimental relevance, independent of the question of the non-asymptotic scaling of the longest relaxation time $\tau_{D}$ with $N$.

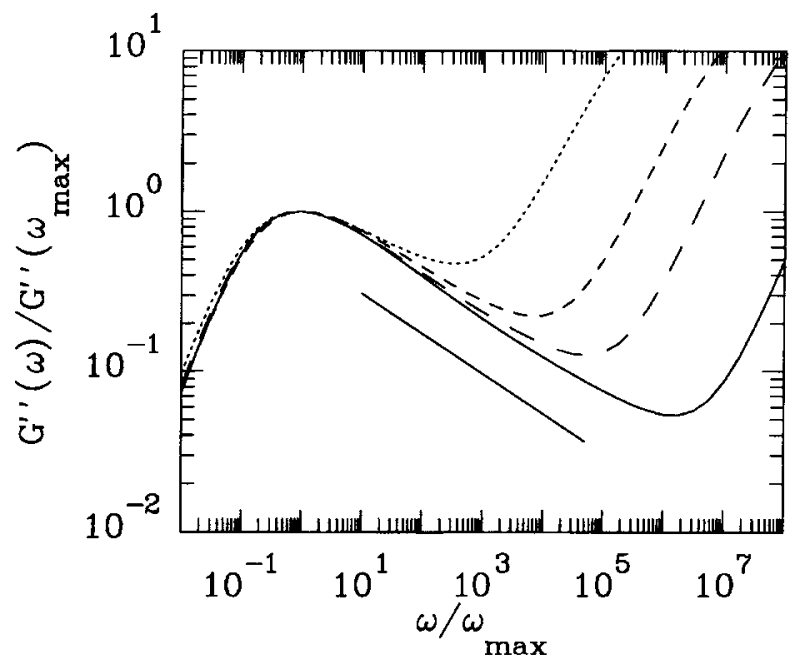

Fig. 22. Normalized stress relaxation loss moduli versus reduced frequency under meltlike conditions for $N / N_{n}$ $=20$ (short dashed), 50 (dashed), 100 (long dashed), and 300 (solid). A solid line of slope $-1 / 4$ is also shown

Model calculations ${ }^{77)}$ also reveal effective $N$-dependent scaling laws for other features of the stress moduli. For example, over the remarkably broad range $N / N_{\eta}=$ $20-1000$, nearly perfect power law scaling is predicted for: $\tan \delta_{\min }=G_{p} / G^{\prime \prime}\left(\omega_{\min }\right)$ $\sim G^{\prime \prime}\left(\omega_{\max }\right) / G^{\prime \prime}\left(\omega_{\min }\right) \sim N^{-0.78}$, and $G_{p} / G^{\prime \prime}\left(\omega_{\max }\right) \cong 4.5 \sim N^{0}$. Here, the plateau modulus is defined as the storage modulus evaluated at the frequency where the loss modulus goes through a minimum, $G_{p}=G^{\prime}\left(\omega_{\min }\right)$. These $N$-scaling laws are in excellent agreement with many old and recent experimental studies ${ }^{1,22)}$, thereby demonstrating that numerical PMC theory can accurately predict the relative $N$-dependence of moduli. The location of the maximum and mimimum of the loss modulus over the range $N / N_{\eta}=20-300$ follow the power laws: $\omega_{\max } \sim N^{-3.1}$ and $\omega_{\min } \sim N^{-1 / 4}$. The predicted exponents correspond to a significantly weaker dependence than found experimentally: $\omega_{\max } \sim N^{-3.5}$ and $\omega_{\min } \sim N^{-0.8 \pm 0.2}$, although the rather shallow frequency minimum is difficult to accurately measure ${ }^{1,22)}$. We believe the poorer agreement between the numerical PMC theory calculations and experiment for $\omega_{\max }$ is due to the neglect of the constraint release mechanism.

The dependence of the terminal relaxation time, plateau modulus, and other properties on reduced polymer density $\rho \sigma^{3}$ found from the numerical calculations are in 
good accord with the analytic results discussed in sections II and III. This is not surprising since Eq. (6.2) and the same PRISM determined equilibrium input was employed. Excellent superposition behavior was also found ${ }^{77)}$. That is, when compared at fixed $N / N_{\eta}$, and plotted in reduced variable format, the $G^{\prime \prime}(\omega)$ and $G^{\prime}(\omega)$ curves for different reduced polymer densities (solutions) collapse almost perfectly onto a master curve, consistent with experimental behavior ${ }^{1)}$.

After calibration of the crossover function in Eq. (6.1), a zero fit parameter comparison of theory and experiments for $G^{\prime}, G^{\prime \prime}$, and the loss tangent of a 2.54 million molecular weight polystyrene melt ${ }^{22)}$ is shown in Figs. 23 and 24. The calculations employ the experimentally relevant value of $N / N_{\eta}=80$. There are no adjustments

Fig. 23. Comparison of PMC predictions (curves) and experiment (open symbols) for the normalized loss and storage moduli as a function of reduced frequency for a high molecular weight polystyrene melt ${ }^{22)}$. The PMC curves are obtained based on the melt-like reduced density and $N /$ $N_{\eta}=80$ appropriate for a $M=2.54 \times 10^{6}$ polystyrene sample with a viscosity crossover mass of $M_{\mathrm{c}} \cong 32000$

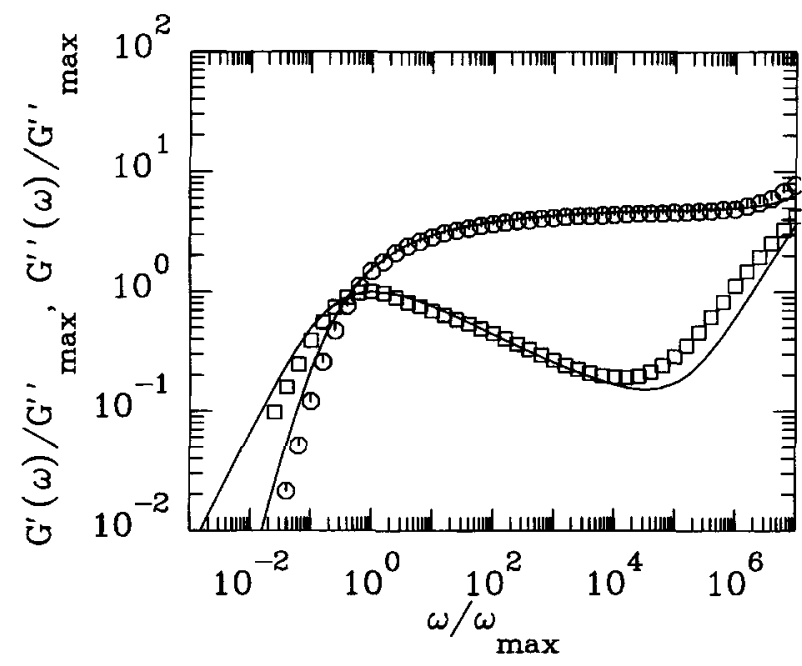

Fig. 24. Same as Fig. 24 but for the loss tangent

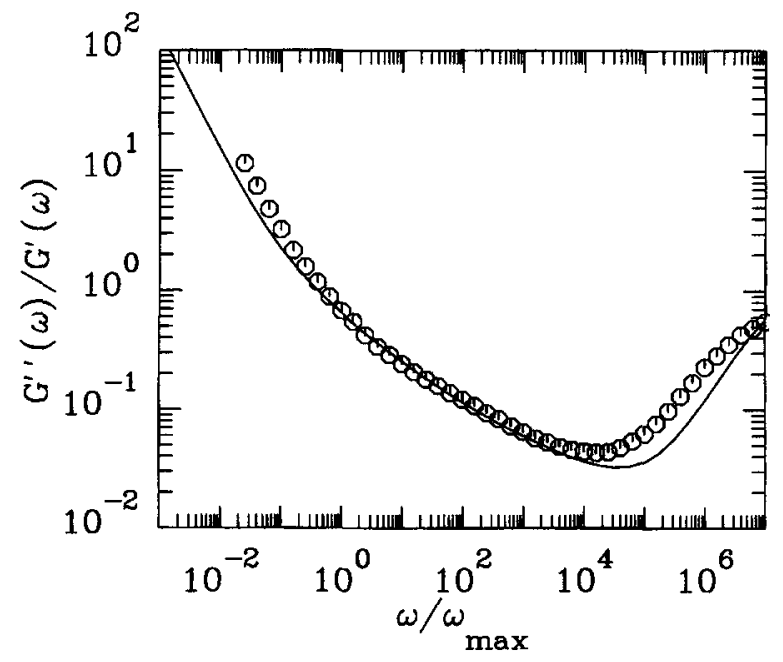


made in the comparisons except to use the reduced variable format. Overall, remarkable agreement between theory and experiment is found over $\cong 9$ orders of magnitude in frequency, which demonstrates the ability of the theory to correctly capture the moduli frequency dependence.

As recently emphasized by Winter ${ }^{22)}$, viscoelastic data at high frequencies $\omega>$ $\omega_{\min }$ show effective power law behavior but with an apparent exponent, $\omega^{0.67}$ roughly. This behavior is not in agreement with the unentangled Rouse law of $\omega^{0.5}$. Such a failure of Rouse theory to describe $G(t)$ in the "pre-entanglement" regime is ubiquitous, having been consistently observed over many years in many different materials. There appears to be a degree of nonuniversality, with exponents spanning the range of $\cong 0.55-0.8$ being reported ${ }^{1)}$. Clearly, Rouse dynamics is significantly perturbed by the "entanglement formation" processes. Qualitatively, our numerical PMC calculations appear to capture this phenomenon, and for the case in Fig. 23, an apparent scaling law of $\cong \omega^{0.8}$ is found. We also caution that our predictions are expected to be less reliable for this shorter time feature due to its enhanced sensitivity to the crossover model employed. Nevertheless, the trend of a considerably larger exponent than the Rouse value is consistently found, and presumably reflects an accelerated self-similiar approach to an elastic state.

Dielectric relaxation has also been numerically studied ${ }^{41,77)}$ based on the assumption it probes the end-to-end vector time correlation function $R(t)$. Fig. 25 shows the dielectric loss function in the terminal regime is much narrower than its viscoelastic analog, in qualitative agreement with experiments ${ }^{20)}$ and the analytic PMC results in section III.

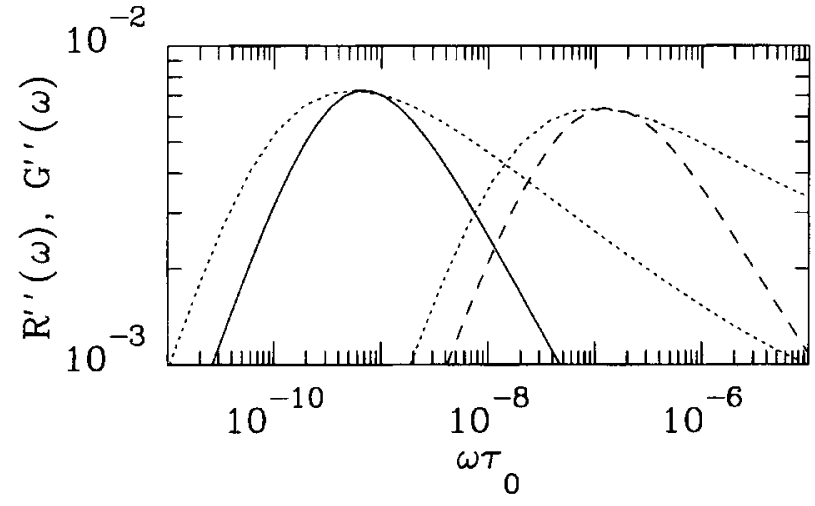

Fig. 25. Comparison of the viscoelastic (short-dashed), "dielectric" (solid and longdashed) loss moduli for $N / N_{\eta}=20$ and 100 and the melt-like reduced density. The dielectric moduli have been shifted to agree with the viscoelastic counterpart at the peak in order to compare spectral shapes

There remain several problems with numerical PMC theory for viscoelastic (and possibly dielectric) properties. (1) As discussed above, a spurious, common $\mathrm{N}$ dependence of the absolute magnitude of moduli amplitudes is predicted for experimentally accessible degrees of polymerization. (2) The terminal relaxation time incorrectly scales very nearly as the $N^{3}$ law. (3) The storage and loss moduli are too broad at low frequencies $\omega<\omega_{\max }$ (see Fig. 23), in a manner which appears to wor- 
sen as $N$ decreases. For example, the overall breadth of the relaxation spectrum is often characterized by the product $G_{p} J_{\mathrm{e}}$, where $J_{\mathrm{e}}$ is the zero frequency limit of $\omega^{-2}$ $G^{\prime}(\omega)$. Values of $G_{p} J_{\mathrm{e}} \cong 4.5$ are found numerically, which significantly exceed typical experimental values ${ }^{1,4,5)}$ of $\cong 2.4-3$.

Problems (2) and (3) clearly arise from the neglect of self-consistent constraint release which, as demonstrated in section IV, decreases the terminal time in an $\mathrm{N}$ dependent manner such that a $N^{3.4}$ law emerges, and cuts off the long time tail of the viscoelastic memory function $M(t)$ which is the origin of the excessive broadening of the low frequency response (see Eq. (4.4)). At the expense of considerable additional effort, self-consistent constraint release can be incorporated in the numerical PMC approach, and this represents an important future direction. The origin of problem (1) is less clear, but may be associated with our crude p-space crossover model, and/or the fact that emergence of an $N$-independent plateau modulus requires $M(t=0) \sim\left(N / N_{\mathrm{e}}\right)^{1 / 2} \gg 1$. Future work is required to attain a clear understanding of this issue.

Other dynamic properties can be investigated using numerical PMC theory ${ }^{77)}$. These include the nonexponential decay of the normal mode autocorrelations $C_{p}(t)$ and their effective relaxation times $\tau_{p}$, coherent and incoherent dynamic structure factors, the frequency-dependent spin-lattice relaxation time $T_{1}(\omega)$, and creep compliance moduli $J_{\mathrm{e}}(\omega)$.

\section{Entangled fractal macromolecules}

Sufficiently dense fluids of large, interpenetrating fractal-like macromolecules invariably display slow dynamics. Global architectures such as chains, rings, starbranched, combs, rods, and even non-fractal microgels or "rubber balls" all display characteristic "entanglement" effects. Recent work by Antonietti and coworkers" has emphasized that observable entanglement features are often surprisingly weakly sensitive to global architecture and the details of connectivity. In particular, melts of chains, rings, and microgel rubber balls display striking similiar entangled viscoelastic behavior. Such observations may suggest there exist rather generic aspects of the entangled dynamics phenomena, contrary to the case-by-case architecture-specific spirit of tube-based phenomenological approaches ${ }^{2,3)}$. Such a possibility has motivated Fuchs and Schweizer ${ }^{47)}$ to generalize and apply the PMC approach to a class of simple Gaussian fractal macromolecules which have been thoroughly studied in their unentangled, Rouse limit by Muthukumar ${ }^{99)}$ and Cates ${ }^{100)}$. Additional motivation is purely theoretical, and in the spirit of renormalization group and mode-coupling studies of dynamic critical phenomena and fracton dynamics ${ }^{53-55)}$. Elucidation of the predictions of PMC theory as a general function of spatial, $d$, and polymer mass fractal, $d_{F}$, dimensions may perhaps shed light on the question of what an entanglement is and how it is relaxed.

The Gaussian fractal model captures only the self-similiar nature of macromolecular density distribution, and not the details of connectivity. Special architectures such as stars and rods display distinctive entanglement behavior due to branching, or chain rigidity and translation-rotation coupling, respectively. Such systems are 
presumably not amenable to a treatment based on a Gaussian fractal macromolecule composed of statistically equivalent sites, and the isotropic cage-averaged PMC dynamical theory ${ }^{47)}$.

\section{A. Basic theoretical aspects}

The intramolecular structure of a single, ideal Gaussian fractal is specified by its size-mass scaling relation and structure factor

$$
\begin{aligned}
& R_{\mathrm{g}} \sim N^{v}, \quad v=1 / d_{\mathrm{F}} \\
& \omega(k)=N^{-1} \sum_{a, \gamma=1}^{N} \exp \left(-k^{2} \sigma^{2}|\alpha-\gamma|^{2 v} / 6\right) \cong \frac{N}{\left(1+c_{v} k^{2} \sigma^{2} N^{2 v}\right)^{1 /(2 v)}}
\end{aligned}
$$

where the constant $c_{v}$ is the same as discussed below Eq. (5.2). The approximate equality in Eq. (7.2) is a convenient interpolation formula which retains all important aspects of fractal macromolecular structure. The Markovian dynamical properties of unentangled fluids of such Gaussian fractals at the Rouse theory level $\operatorname{are}^{99,100)}$

$$
D=\left(\beta \zeta_{0} N\right)^{-1}, \quad \tau_{\mathrm{R}}-N^{1+2 v} \tau_{0}, \quad \eta \sim \zeta_{0} N^{2 v}
$$

Frequency scaling laws for response functions have also been worked out ${ }^{99,100)}$. The Rouse coherent dynamic structure factor can be adequately described with the simple form of Eq. (2.12).

The RR, PMC, and equilibrium PRISM theories at the thread model level have all been generalized to treat Gaussian fractals ${ }^{47)}$. The nontrivial mathematical analysis required to generalize the effective continuum GLE's of Eq. (2.25), and their (approximate) diagonalization by normal modes, has been carefully worked out. The questions of slow bilinear variable choice and memory functions have been treated at the simplest, original level of PMC theory ${ }^{36)}$ which ignores self-consistent constraint release and constraint porosity corrections. Thus, only the large $N$, asymptotic analytic behavior can be reliably investigated. Although predictions have been derived for the polymer solution density dependence in good and theta solvents ${ }^{47 \text { ), }}$ here we focus only on results for melt-like conditions.

In qualitative terms, modifications of the PMC predictions for entangled dynamics due to variable fractal architecture and spatial dimension arise from two distinct physical effects, one global and one local. (i) The number of contacts between a pair of interpenetrating fractals is proportional to the geometric factor $N^{\theta}$, where $\theta=2-d v$. For $d_{\mathrm{F}}<d / 2$ this number is not significant and PMC theory predicts entanglements effects are irrelevant and Rouse dynamics remains valid. (ii) The strength of interchain excluded volume forces is controlled by local segmental scale packing in the dense fluid which depends on dimensionalities and is predicted by PRISM theory. For the $d v<2$ entangled case, effects (i) and (ii) strongly compete. That is, as the fractal dimension increases and/or spatial dimension decreases, 
the number of global contacts between a pair of interpenetrating macromolecules, and hence the geometric aspects of entanglement coupling, are enhanced. However, for these same conditions the densification of the macromolecule causes the intermolecular pair correlation function at the contact, $g(\sigma)$, to strongly decrease even under the experimentally relevant constant pressure or isothermal compressibility condition $^{47)}$. Since the former geometric effect is $N$-dependent and controls the long range intermolecular correlation hole, but $g(\sigma)$ is an intrinsic $N$-independent measure of the strength of intermolecular excluded volume forces, consideration (i) will dominate or "win" in the (hypothetical) $N \rightarrow \infty$ limit for interpenetrating macromolecules. However, for experimentally accessible values of $N$, subtle behavior can emerge due to this competition. For finite frequency response functions, power law scaling exponents will also be sensitive to the precise self-similiar nature of the macromolecule and the intermediate scale packing correlations.

\section{B. Asymptotic predictions and model calculations}

Fig. 26 presents model calculations of the crossover degree of polymerization signaling the breakdown of Rouse theory and entanglement onset, as a function of polymer fractal dimension in $d=3$. The fractal macromolecular analogs of the two RR crossover criteria of Eqs. (2.16) are utilized ${ }^{47)}$. A crossover $N_{\mathrm{c}}^{\prime}$ is calculated from the Markovian condition that $\Delta \zeta=a \zeta_{0}$, with the numerical prefactor " $a$ " chosen so that the value obtained for $d_{\mathrm{F}}=2$ linear chains is $N_{\mathrm{c}}^{\prime} \cong 35$, as found in melt computer simulation studies ${ }^{31,78)}$. A second measure of crossover, $N_{\mathrm{c}}$, is computed using the time domain relation for $\tau_{\mathrm{c}}$ and the fractal-generalized Rouse connection between time and displacement. Analytic string PRISM theory ${ }^{60)}$ is employed to compute the interaction parameter $\psi=\rho \sigma^{3} g^{2}(\sigma) S_{0}$ which is proportional to the segmental scale mean square excluded volume force exerted by the matrix polymers on a tagged segment. To make a "fair" comparison, a calibration procedure was adopted whereby the coarse-grained density $\rho \sigma^{3}$ is chosen to ensure a value of dimensionless liquid

Fig. 26. Crossover degrees of polymerization under the conditions described in the text. From the RR crossover criterion of Eq. (2.16) in the time domain, $N_{\mathrm{c}}$, and Markovian limit, $N_{\mathrm{c}}^{\prime}$. From the PMC theory for self-diffusion, $N_{D}$, and viscosity, $N_{\eta}$, based on Eq. (7.6)

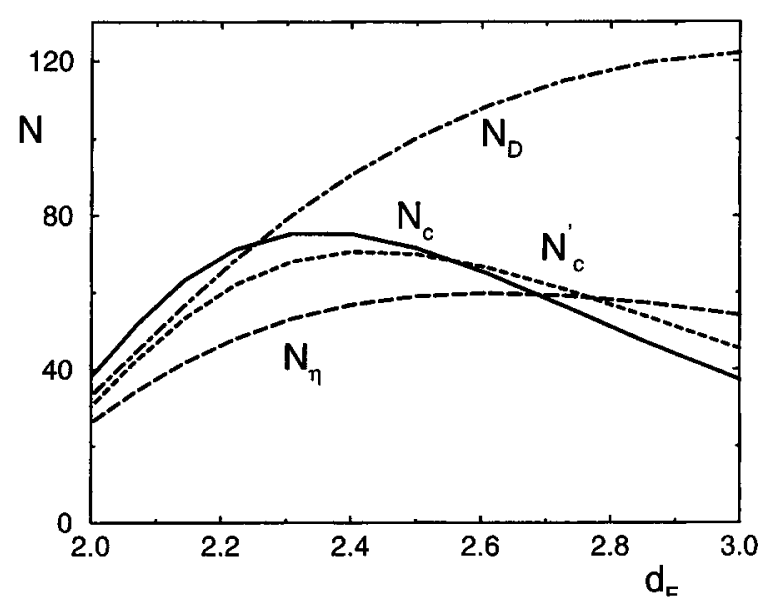


density fluctuations, $S(0)=S_{0}$, which is independent of fractal dimension, thereby crudely mimicking the expected constant pressure equilibrium behavior ${ }^{47)}$.

Nonmonotonic variation of the crossover $N$ 's with fractal dimension is predicted arising from the monotonic variation of the equilibrium parameters for different $d_{\mathrm{F}}$. However, both estimates of a crossover degree of polymerization are rather close to each other, and vary by less than a factor of 3 as a function of fractal dimension. This behavior reflects the subtle competition of the two physical factors discussed above. Moreover, this prediction seems in qualitative accord with experiments ${ }^{6,9)}$ on melts of polystyrene chains $\left(d_{\mathrm{F}}=2\right)$, rings $\left(d_{\mathrm{F}} \cong 2.5\right.$ has been suggested based on heuristic theoretical arguments ${ }^{101)}$ and lattice model simulations ${ }^{102}$ ), and dense "microgel-like" systems $\left(d_{\mathrm{F}}=3\right)$, in the sense that entangled behavior emerges for degrees of polymerization which vary only by roughly a factor of 2 or 3 . Note that a $d_{\mathrm{F}}=3=d$ interpenetrating fractal model is not a faithful description of the non-fractal crosslinked "rubber balls"9), but is the best one can do based on the simple fractal macromolecular model adopted. Although both "spherical microgels" are dense in the sense $R_{\mathrm{g}} \sim N^{1 / 3}$, the fractal macromolecule is a porous structure with a fuzzy surface since $\omega(k) \sim k^{-3}$ for intermediate scales $\sigma^{-1} \ll k \ll R_{\mathrm{g}}^{-1}$, while the nonfractal "rubber balls" are not porous and show a $\omega(k) \sim k^{-4}$ Porod scattering regime indicative of a sharply defined surface ${ }^{9)}$.

In the $N \rightarrow \infty$ asymptotic limit, PMC theory scaling predictions for the terminal relaxation time and transport coefficients for $d v<2$ are given by ${ }^{47)}$

$$
\tau_{D} \sim g^{2}(\sigma) N^{5-2 v(d-1)}, \quad D \sim\left(g^{2}(\sigma) N^{5-2 v d}\right)^{-1}, \quad \eta=\frac{\rho k_{\mathrm{B}} T}{N_{\mathrm{e}}(v, d)} \tau_{D}
$$

where $N_{\mathrm{e}}$ is determined from the RR crossover theory, and $g(\sigma)$ is also a function of fractal and spatial dimensionalities. The entanglement enhancement of the effective friction constant per segment again is proportional to $\left[g(\sigma) N^{2-d \nu}\right]^{2}$, the square of the product of the local strength of intermolecular hard core forces times the geometric factor quantifying the number of contacts between a pair of interpenetrating macromolecules. Note that larger exponents are predicted for more compact fractal architectures: $D \sim N^{-2}, N^{-2.6}, N^{-3}$ and $\eta \sim N^{3}, N^{3.4}, N^{3.67}$ as $d_{\mathrm{F}}$ increases from 2 (chains) to 2.5 (rings) to 3 . However, the "Stokes-Einstein" ratio, $D \tau_{D} / R_{\mathrm{g}}^{2}$, is found to be nearly constant in 3-dimensions ${ }^{47)}$, increasing monotonically by a factor of less than three as the macromolecule fractal dimension increases from 2 to 3 . This is a nontrivial result which originates from the common mechanism for entanglement force relaxation at the memory function level which controls both center-of-mass diffusion and conformational dynamics and stress relaxation.

It is interesting to contrast the PMC predictions of Eq. (7.4) with the analogs based on a reptation/tube approach. If the Gaussian fractals can be crudely thought of as linear flexible polymers, then the most naive generalization of the tube model ansatz predicts for all spatial dimensions

$$
\tau_{D}^{\text {rep }} \sim \frac{N^{2}}{D^{\mathrm{R}}} \sim N^{3} \tau_{0} \Rightarrow D^{\text {rep }} \sim \frac{R_{\mathrm{g}}^{2}}{\tau_{D}} \sim N^{-3+2 v}
$$


Curiously, this result (accidentally) agrees with Eq. (7.4) in $d=3$ only for the $d_{\mathrm{F}}=2$ ideal chain case. PMC theory also predicts a significant dependence of the entanglement friction on spatial dimension. For example, in a $d=2$ melt of self-avoiding random coils $\left(d_{\mathrm{F}}=4 / 3\right)$, PMC predicts $\tau_{D} \sim N^{3.5}$ and $D \sim N^{-2}$, the latter in accidental agreement with the $d=3$ reptation result. This case is directly relevant to recent simulations of self-avoiding chains in a dense 2 -d obstacle net ${ }^{96)}$.

To address real systems, the Rouse-entangled crossover and possible strong finite $N$ corrections must be taken into account. The latter analysis has not yet been carried out for the general fractal polymer problem. However, as a crude approach a naive crossover model can be adopted where the bare and large $N$ results are simply added $^{47)}$

$$
\frac{D^{\mathrm{R}}}{D}=1+\left(\frac{N}{N_{D}}\right)^{4-2 d v}, \quad \frac{\eta}{\eta^{\mathrm{R}}}=1+\left(\frac{N}{N_{\eta}}\right)^{5-2 d v}
$$

The crossover degrees of polymerization can be computed based on the RR, PMC, and PRISM generalizations to the fractal macromolecule case ${ }^{47)}$. The resulting values for $d=3$ are included in Fig. 26. More compact fractal macromolecules follow Rouse-like self-diffusion up to higher $N$ values again reflecting the subtle competition between "prefactor", $g(\sigma)$, and "geometric" (number of contacts $N^{2-3 v}$ ) contributions to the center-of-mass friction. On the other hand, the viscosity crossover and $N_{\mathrm{c}}$ are similiar.

Model calculations for the $N$-dependence of $D$ and $\eta$ for the chain, ring, and microgel-like values of fractal dimension are shown in Figs. 27 and 28. For self-diffusion, in the experimentally accessible window of $N / N_{D}<100$, the differences in reduced diffusion constant is less than half a decade and "curve crossings" occur. Only for very high molecular weights do the enhanced large $N$-scaling exponents for the more compact macromolecules dominate over the reduced prefactor $g(\sigma)$. Similar trends are also found for the shear viscosity, with dominance of the geometric $N$ -

Fig. 27. Logarithmic plot of the reduced selfdiffusion coeffients versus degree of polymerization for several fractal dimensions in $d=3$. The vertical lines mark the crossover degrees of polymerization, $N_{D}$, from Fig. 26

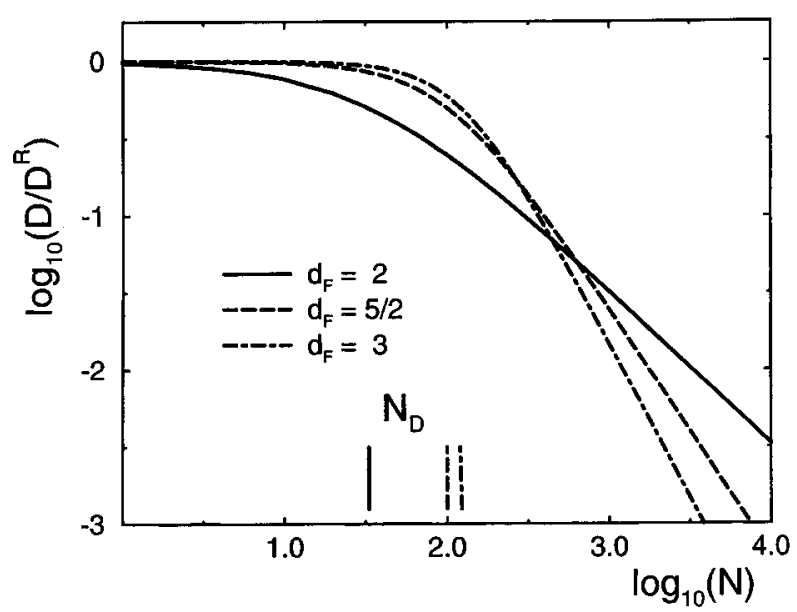




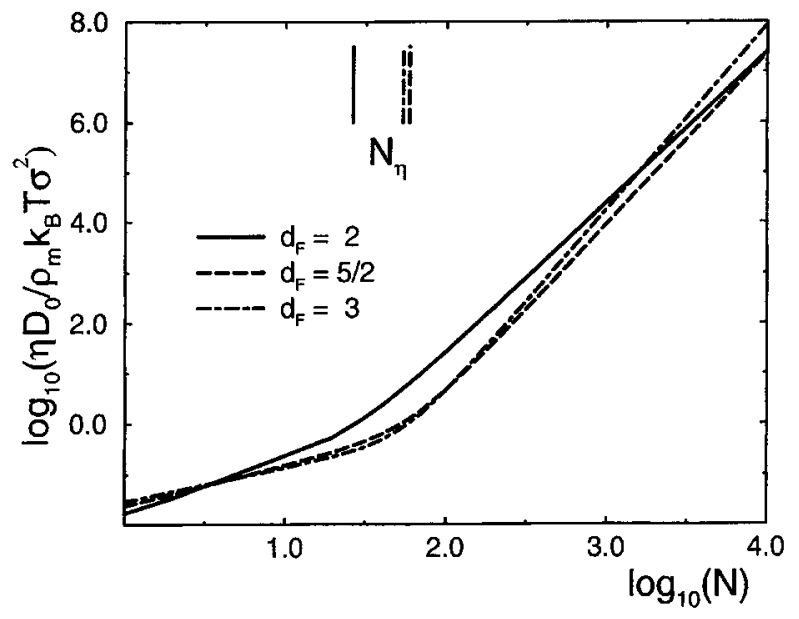

Fig. 28. Logarithmic plot of the reduced shear viscosities versus degree of polymerization for several fractal dimensions in $d=3$. The vertical lines mark the crossover degrees of polymerization, $N_{\eta}$, from Fig. 26

scaling factor being delayed until even larger degrees of polymerization than found for translational diffusion. These model calculations would seem to be in rough qualitative agreement with shear viscosity measurements on polystyrene chain, ring, and microgel melts ${ }^{6,9)}$. The results in Fig. 27 also suggest there is a regime (where viscosity scales approximately as $N^{3.5}$ ) where the self-diffusion constant of $d_{\mathrm{F}}=3$ entangled macromolecules can be an order of magnitude smaller than its linear chain counterpart, even though their shear viscosities are nearly identical.

Predictions for a host of intermediate frequency and time properties as a general function of space and fractal dimensions have been derived ${ }^{47)}$. Here, we summarize a few of these results, emphasizing the dependence on fractal dimension in the most experimentally relevant range $d_{\mathrm{F}}=2-3$ in $d=3$.

Anomalous mean square segmental diffusion in the first (broadest) intermediate time regime is predicted to follow the power law

$$
\left\langle(\boldsymbol{r}-\boldsymbol{r}(t))^{2}\right\rangle \sim t^{2 v(v(d+2)-1)^{2} /(1+2 v)^{3}}, \quad \tau_{\mathrm{C}} \ll t \ll \tau_{\mathrm{R}}
$$

The intermediate time segmental (and center-of-mass) diffusion is increasingly slowed as the macromolecule becomes more compact. For example, in 3-dimensions Eq. (7.7) yields: $t^{9 / 32}\left(d_{\mathrm{F}}=2\right), t^{20 / 81}\left(d_{\mathrm{F}}=2.5\right)$, and $t^{8 / 125}\left(d_{\mathrm{F}}=3\right)$.

Predictions for the viscoelastic loss modulus in the pre-terminal intermediate frequency regime are

$$
\begin{array}{rlrl}
G^{\prime \prime}(\omega) & \sim \omega^{-x}, & x & =(2-d v)(v(d+2)-1) /(1+2 v)^{2} \quad \tau_{\mathrm{R}}^{-1}<\omega<\omega_{\min } \\
\sim \omega^{-x}, & x & =(2-d v) /(1+2 v) \quad \tau_{\mathrm{RR}}^{-1}<\omega<\tau_{\mathrm{R}}^{-1}
\end{array}
$$

Interestingly, in the broadest (first) intermediate regime a nearly universal power law exponent is predicted, $x=0.2 \pm 0.05$, for $2<d_{\mathrm{F}}<3$. In the final intermediate regime, the apparent exponent increases significantly (less dissipation) as the macro- 
molecules become more compact: $x=1 / 4\left(d_{\mathrm{F}}=2\right), x=4 / 9\left(d_{\mathrm{F}}=2.5\right)$, and $3 / 5\left(d_{\mathrm{F}}=\right.$ 3 ). The $N$-dependence of the viscoelastic response of the minimum dissipation state follow the scaling laws

$$
\begin{aligned}
\omega_{\min } \sim N^{-y}, \quad G^{\prime \prime}\left(\omega_{\min }\right) \sim \tan \delta_{\min } \sim N^{-y /(1+2 v)} \\
y=\frac{2(2-d v)(1+2 v)^{2}}{(1+2 v)+(2-d v)(v(d+2)-1)}
\end{aligned}
$$

Enhanced $N$-dependence of the loss tangent and frequency minimum are predicted with increasing fractal dimension. For example, in $d=3$ one obtains for ideal Gaussian coils $G^{\prime \prime}\left(\omega_{\min }\right) \sim \omega_{\min }^{1 / 2} \sim N^{-8 / 11}$, whereas $G^{\prime \prime}\left(\omega_{\min }\right) \sim \omega_{\min }^{3 / 5} \sim N^{-10 / 7}$ is found for $d_{\mathrm{F}}=3$. These predictions for the viscoelastic response are possibly testable by systematic experiments on melts of rings, or perhaps (more speculatively) on entangled microgels ${ }^{9)}$. Model calculations of finite frequency viscoelastic moduli have been presented elsewhere ${ }^{47)}$.

We believe the predictions of PMC theory for entangled fractal macromolecular fluids do reveal a generality to the entanglement phenomenon for certain architectures which supports recent suggestions of Antonietti and coworkers ${ }^{9)}$. More precise comparison with experiments may require the proper treatment of the finite size corrections and/or the numerical aspects discussed in sections IV and VI. A thorough experimental study of the viscoelastic, diffusion, and equilibrium structural properties of highly entangled polymer ring melts would seem the best experimental system to test the theory. Finally, neglect of certain connectivity aspects is no doubt inadequate for some architectures such as highly branched polymers and rigid rods, although a promising microscopic statistical dynamical approach for rods has been recently developed by Szamel ${ }^{103)}$. Finally, we note that a convincing PMC theory treatment of the non-fractal, microgel "rubber balls" also remains to be achieved.

\section{Blends and block copolymers}

The collective dynamics of any binary $\mathrm{AB}$ mixture, atomic or macromolecular, undergoes critical slowing down as a second order phase transition is approached ${ }^{54)}$. The reason is the emergence of low amplitude, long wavelength concentration fluctuations, which diverge at a critical point. The real space picture is physical clustering of like species, corresponding to the formation of diffuse interfaces between $\mathrm{A}$ and B species and domains or droplets of like species. Properties such as the collective diffusion constant, shear viscosity, and low frequency viscoelastic response all show anomalous critical behavior. Rheological anomalies and slowing down of collective dynamics are also expected and observed in self-assembling block copolymer fluids ${ }^{12)}$, where much larger amplitude concentration fluctuations emerge near the order-disorder transition (ODT) corresponding to microphase separation of A and B species on a finite length scale commensurate with macromolecular size, $R_{\mathrm{g}}$.

There is another question. Is there an influence of long wavelength concentration fluctuations and thermodynamically-driven physical clustering on the single polymer translational diffusion and conformational relaxation? For polymers the answer 
may depend on whether the chains are entangled or not. Since PMC theory envisions entanglement dynamics as having a structural origin (long range correlation hole), one might anticipate that entangled polymer dynamics will be strongly perturbed by long wavelength concentration fluctuations, but unentangled polymers may not, or at least be much less, affected. Since the polymer size and microdomain length scales are nearly identical for diblock copolymers, one expects much larger effects in entangled copolymers than for the corresponding blends. The highly segregated diblock copolymer problem may also be related to slow dynamics in confined polymer fluids, and tethered chain systems such as polymer brushes.

Recent diffusion and viscoelastic experiments on symmetric diblock copolymers $^{12,104-106)}$ and binary blends ${ }^{107,108)}$ seem to qualitatively agree with the above scenario. Theoretical work by Tang, Schweizer and Guenza ${ }^{44-46)}$ for entangled fluids based on the simplest original version of the PMC approach ${ }^{36)}$, and by Genz and Vilgis ${ }^{109)}$ using PMC theory for the unentangled case, are also in accord with this scenario. The basic physical idea is schematically illustrated in Fig. 29 for the case of a diblock copolymer fluid. Effective forces, correlated in space and time, are exerted by the surrounding multicomponent matrix on two tagged chain segments which are mediated by intrachain and collective (density and concentration) fluctuations. The polymer-matrix interactions can be of two origins: the hard core excluded volume force which dominates entanglement in one-component melts, and the "tail" (generally attractive) forces between species which control the enthalpy of mixing and are traditionally quantified as a single net $\mathrm{AB}$ interaction or $\chi$ parameter. Anticipating the conclusions deduced below, since $\chi$ is constrained to be of order $N^{-1}$ in the homogeneous phase of interest its direct contribution to friction is generally small for high molecular weight entangled fluids, and to a good approximation can be ignored entirely. This conclusion is supported by the PMC analysis of Genz and Vilgis for unentangled blends and diblock fluids which was based on a bare Rouse description of memory function dynamics ${ }^{109}$. The only caveat appears to be for blends extremely close to the critical point where the $k=0$ divergence of concentration fluctuations can induce nonintegrable, long time tails in the viscoelastic mem-

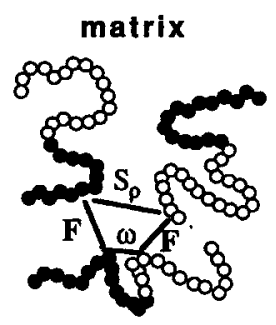

tracer

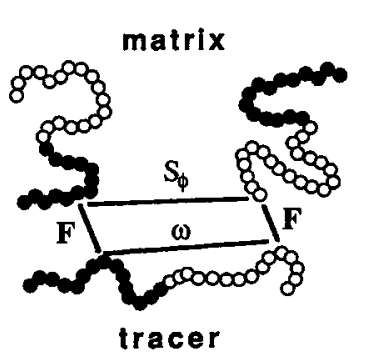

Fig. 29. Illustration of the force correlations in RR and PMC theory for multicomponent systems. The case of an $\mathrm{AB}$ diblock copolymer fluid is shown, where $F$ denotes an effective repulsive force, $\omega$ is the intrachain correlation, and $S_{\rho}$ and $S_{\phi}$ are the matrix collective density (short range) and concentration (long range) fluctuations, respectively 
ory function associated with the $\chi$ forces. Such long time tails may (or may not) have observable consequences for shear viscosity and low frequency moduli extremely close to a critical point ${ }^{109}$ ).

Despite its direct dynamical insignificance for entangled polymers, $\chi$ controls the local and macromolecular scale equilibrium physical clustering and (micro)domain formation. The latter provides constraints on the ability of the hard core entanglement forces to relax. Since PMC theory describes entanglement force relaxation using the RR model, generalization of the Renormalized Rouse approach is the crucial technical task required to treat entangled blends and diblock fluids.

Although algebraically complicated, generalizations to binary AB polymer fluids (blends or diblocks) of the RR and PMC approaches can be achieved ${ }^{44-46)}$. Published work has considered mainly the simplest possible model with the maximum number of symmetries. The A and B chains are taken to obey ideal Gaussian statistics, with $\mathrm{A}$ and $\mathrm{B}$ sites of identical hard core diameter and segment length $\sigma$, bare friction constant $\zeta_{0}$, and equimolar composition of $\phi=0.5$ (blends) or $f=0.5$ (diblocks). The $A$ and $B$ species are distinguished only by a weak interchain tail potential associated with an effective $\chi$ parameter.

In this section the central novel features which emerge from RR and PMC theory for symmetric models of entangled blends and block copolymers in the dense melt state are sketched. We focus primarily on the mathematically simplest property, the center-of-mass self-diffusion constant $D$. The technical simplifications appropriate for large $N$ and melt density conditions, i.e. neglect of constraint release and porosity corrections within the naive frozen matrix framework of the original PMC formulation ${ }^{36)}$, are employed. Generalizations to treat tracer diffusion, chain dynamics, the unentangled-entangled crossover, matrix constraint release and porosity, friction constant and chain length asymmetries, and the effects of solvent dilution have been partially worked out $\left.{ }^{44} 46,109\right)$ and represent directions of work in progress ${ }^{110}$. We believe that generalization of PMC theory to multicomponent, phase separating fluids presents an exciting opportunity to test the unique ability of a microscopic theory to provide precise connections between structure and dynamics. Liquid-state integral equation theories (PRISM) of the structure, thermodynamics, and phase behavior of blends and block copolymers provide crucial input, and have been extensively reviewed recently ${ }^{57}$.

\section{A. Theory of self-diffusion}

Consider first the composition-dependent self diffusion constant, $D^{\mathrm{p}}$, of species " $p$ " in a binary $A B$ homopolymer mixture ${ }^{45)}$. For entangled systems the effective friction coefficient, $\zeta^{\mathrm{p}}$, is the bare monomeric contribution plus the time integral of the correlation of the segmental scale intermolecular excluded forces exerted on the probe center-of-mass. The latter is computed based on the PMC approach, where there are two slow bilinear variables associated with the product of the single probe polymer density and the collective density of species $\mathrm{M}=\mathrm{A}, \mathrm{B}$ of the surrounding matrix. For polymer alloys close to a phase separation transition, the latter collective variables are expected to be particularly slow, and neglect of matrix constraint 
release is especially appropriate. For simplicity, constraint porosity corrections are also ignored. The resulting PMC theory expressions based on these simplifications $\operatorname{are}^{45)}$

$$
\begin{aligned}
D^{\mathrm{p}} & =\left(\beta \zeta^{\mathrm{p}} N_{\mathrm{p}}\right)^{-1} \\
\zeta^{\mathrm{p}} & =\zeta_{0}^{\mathrm{p}}+\left(\rho /\left(6 \pi^{2} \beta\right)\right) \int_{0}^{\infty} \mathrm{d} t \int_{0}^{\infty} \mathrm{d} k k^{4}\left\{\sum_{\mathrm{M}, \mathrm{M}^{\prime}} C_{\mathrm{pM}}(k) S_{\mathrm{MM}^{\prime}}(k) C_{\mathrm{M}^{\prime} \mathrm{p}}(k)\right\} \omega_{\mathrm{p}}^{\mathrm{RR}}(k, t) \\
& =\zeta_{0}^{\mathrm{p}}+\left(-C_{0} /\left(6 \pi^{2} \beta\right)\right) \int_{0}^{\infty} \mathrm{d} t \int_{0}^{\infty} \mathrm{d} k k^{4} \omega_{\mathrm{p}}^{\mathrm{RR}}(k, t)
\end{aligned}
$$

In the first line of Eq. (8.2) the subscripts refer to the species type (A or B), and the terms in large braces represent the "medium-induced potential of mean force" between segments on the probe chain due to interactions with the surrounding blend matrix. The second line follows from the weakness of the $\chi$ parameter forces, and the reduction of equilibrium structural properties to a homopolymer reference melt (denoted by subscript " 0 ") in the $\chi \rightarrow 0$ limit. It is identical to the corresponding homopolymer expression except the probe RR dynamics must be evaluated in the presence of both total density and concentration fluctuations.

The single chain RR propagator is evaluated using Eq. (2.33). The appropriate $R R$ effective friction constant is the blend generalization of Eq. (2.11)-(2.13) and is given by ${ }^{45)}$

$$
\begin{aligned}
\zeta_{\mathrm{RR}}^{\mathrm{p}} & =\zeta_{0}^{\mathrm{p}}+\Delta \zeta_{\mathrm{p}} \\
\Delta \zeta_{\mathrm{p}} & =\frac{8}{3} \beta^{-1} \sigma^{4} \rho \int_{0}^{\infty} \mathrm{d} t \int_{0}^{\infty} \mathrm{d} k k^{2} j_{1}^{2}(k \sigma)\left\{\sum_{\mathrm{P}, \mathrm{P}} g_{\mathrm{PP}}(\sigma) S_{\mathrm{PP}}(k) g_{\mathrm{P} \mathrm{P}}(\sigma)\right\} \omega_{\mathrm{p}}^{\mathrm{R}}(k, t) \\
& =\frac{8}{3} \beta^{-1} \sigma^{4} \rho \int_{0}^{\infty} \mathrm{d} t \int_{0}^{\infty} \mathrm{d} k k^{2} j_{1}^{2}(k \sigma)\left\{4 g_{0}^{2}(\sigma) S_{0}+\left[\Delta g_{\mathrm{p}}(\sigma)\right]^{2} S_{\phi}(k)\right\} \omega^{\mathrm{R}}(k, t) \\
& \sim \zeta_{0} \rho g_{0}^{2}(\sigma) S_{0} \sqrt{N}\left\{1+\lambda J_{\mathrm{p}}\right\}
\end{aligned}
$$

In the second line of Eq. (8.4), the two terms in braces describe the contributions of density (short range) and concentration (long range) fluctuation mediated excluded volume forces, respectively. The former are identical to the pure one-component entangled melt case and are blend composition independent. As indicated in Fig. 29, concentration fluctuations are spatially correlated over large distances, and hence can induce dynamical correlations of the tagged polymer segments which are widely separated in space. Droplet formation enters the force time correlations via local physical clustering of like segments, as quantified by the positive contact value of $\Delta g_{\mathrm{A}}=g_{\mathrm{AA}}-g_{\mathrm{AB}}$ or $\Delta g_{\mathrm{B}}=g_{\mathrm{BB}}-g_{\mathrm{AB}}$, and the concentration fluctuation structure factor $S_{\phi}(k)$. For compositionally symmetric blends $\Delta g_{\mathrm{A}}=\Delta g_{\mathrm{B}}=\Delta g$. However, for compositionally asymmetric mixtures there are distinct measures of minority and majority species local clustering. In PRISM theory for symmetric chain models, the random phase approximation (RPA) form applies: $1 / S_{\phi}(k)=\{\phi(1-\phi) \omega(k)\}^{-1}-2 \chi$, 
where $\phi$ is the volume-fraction of A segments. $\chi$ is fluctuation-renormalized, increases nearly linearly with $T^{-1}$, and depends parabolically (in an $N$-dependent manner) on blend composition ${ }^{57,111)}$.

The final proportionality in Eq. (8.4) implicitly defines the species-dependent function $J_{\mathrm{p}}=J_{\mathrm{p}}(T, N, \phi, p)$ as the ratio of the concentration-fluctuation-mediated to density-fluctuation-mediated friction constants. By symmetry, $J_{\mathrm{A}}(\phi)=J_{\mathrm{B}}(1-\phi)$. A numerical (fudge) factor " $\lambda$ " has been introduced to emphasize the inability to a priori compute the absolute magnitude of the friction. In practice, such a $(N$-independent) numerical factor is fixed by comparison with experimental data. The leading factor in Eq. (8.4) is the pure entangled melt contribution. Using Eqs. (8.1)(8.4) yields for entangled blends

$$
D^{\mathrm{p}}=\frac{N_{D}}{\beta \zeta_{0} N_{\mathrm{p}}^{2}\left(1+\lambda J_{\mathrm{p}}\right)} \equiv \frac{D_{0}}{1+\lambda J_{\mathrm{p}}}, \quad N>N_{D}
$$

where $D_{0}$ is defined as the self-diffusion constant of a reference homopolymer melt unaffected by concentration fluctuations $\left(\chi \rightarrow 0\right.$ limit). The factor $\left(1+\lambda J_{\mathrm{p}}\right)$ also represents the enhancement of the longest single chain terminal relaxation time of species $\mathrm{p}, \tau_{D, \mathrm{p}}$, due to slowing down of the entanglement force time correlations associated with concentration fluctuations. For the symmetric model, this connection follows since PMC theory predicts a "Fick's law" type relation ${ }^{47,109)}, \tau_{D, p} \sim R_{\mathrm{g}}^{2} / D^{\mathrm{p}}$.

The analysis for diblock copolymers proceeds in a formally identical manner ${ }^{44,46)}$. Since the A and B segments are chemically bonded in a diblock, there is only a single self-diffusion constant, $D$. For the highly symmetric $\mathrm{AB}$ model of present interest, Eqs. (8.1)-(8.5) again apply for a $f=1 / 2$ compositionally symmetric diblock copolymer fluid where $2 \Delta g=g_{\mathrm{AA}}+g_{\mathrm{BB}}-2 g_{\mathrm{AB}}$. The only difference with respect to the blend case is that the equilibrium quantities describing concentration fluctuations and physical clustering, $\Delta g$ and $S_{\phi}(k)$, are strongly modified. This is because microdomain scale phase separation occurs on a length scale $D^{*}=2 \pi / k^{*} \cong 3 R_{\mathrm{g}}$, in contrast to macroscale phase separation at $k=0 . k^{*}$ represents the wavevector of maximum instability where $S_{\phi}(k)$ has a sharp peak. The RPA form is $S_{\phi}(k)=(F(k)-$ $2 \chi)^{-1}$, where $F(k)$ is a known ${ }^{12,113)}$ combination of single copolymer partial structure factors and obeys $F\left(k^{*}\right) \sim N^{-1}$.

In contrast with blends where $S_{\phi}(k=0) \rightarrow \infty$ at the critical point or along a spinodal, in diblock fluids the finite wavevector concentration fluctuations destroy all mean field spinodal and critical divergences ${ }^{112,113)}$. The analytic PRISM theory of David and Schweizer ${ }^{114)}$ has been employed to describe the fluctuation renormalization effect on $\chi$ and $S_{\phi}(k)$. Guenza and Schweizer have recently demonstrated that this microscopic liquid state theory provides predictions in excellent agreement with disordered phase experiments in melts and solutions ${ }^{115)}$. In addition, for symmetric diblock melts near and above the order-disorder transition (ODT), PRISM theory predictions are (accidentally) in good qualitative accord with the field theoretic approach of Fredrickson and Helfand ${ }^{113)}$. Empirical estimation of the ODT within the globally isotropic PRISM description can be done rather accurately as discussed elsewhere ${ }^{115)}$. 


\section{B. Analytic predictions and model calculations for blends}

Analytic predictions have been derived by Tang and Schweizer ${ }^{45}$ for the two blend self-diffusion constants based on Eqs. (8.1)-(8.4) plus the known dependences of $\Delta g$ and $S_{\phi}(k)$ on $T, N$, and $\phi$. Here we consider the chain length symmetric case $N_{\mathrm{A}}=N_{\mathrm{B}}=N$, although generalization is straightforward ${ }^{45)}$. Only two results are required for a qualitative understanding of the basic predictions of PMC theory for self-diffusion in blends. (i) On the macromolecular scale, the concentration fluctuation structure factor is proportional to $N$, and tends to a (integrable) $k^{-2}$ divergence at $k=0$ as the blend approaches its spinodal at $2 \phi(1-\phi) \chi_{s} N=1$, where $\chi_{\mathrm{s}}$ is the value of the chi-parameter at the spinodal. Explicitly one has:

$$
S_{\phi}(k)=\frac{2 N \phi(1-\phi)}{\left(k R_{\mathrm{g}}\right)^{2}+2 \phi(1-\phi) N\left(\chi_{\mathrm{s}}-\chi\right)}, \quad \text { where } \quad 2 \phi(1-\phi) \chi_{\mathrm{s}} N=1
$$

(ii) The local clustering functions are identically zero in the high temperature limit where $\chi=0$. As the blend is cooled, clustering of like segments grows monotonically, and in compositionally asymmetric mixtures is much larger for the minority species than the majority species. The qualitative form, for blend compositions $\phi_{\mathrm{p}}$ far above the semidilute crossover concentration and reduced total densities of order $N^{0}$, is ${ }^{45)}$

$$
\Delta g_{\mathrm{p}} \sim \frac{1}{\sqrt{N} \rho \sigma^{3} \phi_{\mathrm{p}}}\left[1-\sqrt{1-\frac{\chi}{\chi_{\mathrm{s}}}}\right]
$$

where $\phi_{\mathrm{A}}=\phi$ and $\phi_{\mathrm{B}}=1-\phi$. Note that the degree of local clustering is a finite size effect proportional to the ratio of the density screening length, $\xi_{\rho} \sim\left(\rho \sigma^{3}\right)^{-1}$, to radius-of-gyration length scales. Moreover, clustering of species $p$ increases monotonically as its volume fraction decreases.

Based on the above results, one can conclude that on the $R_{\mathrm{g}}$ length scale which controls the $N$-dependent entanglement friction contributions, the product $\left(\Delta g_{\mathrm{p}}\right)^{2} S_{\phi}$ and the function $J_{\mathrm{p}}$ in Eq. (8.4) are of order $N^{0}$, and quantitatively increase as the blend approaches phase separation. Thus, concentration fluctuations decrease the self-diffusion constant as the entangled blend approaches its spinodal. However, at fixed thermodynamic state the $\mathrm{N}$-scaling law is not modified. This prediction is consistent with experimental observation ${ }^{107,108)}$ of the $D \sim N^{-2}$ scaling law in some polymer blends far from phase separation. However, since $J$ quantitatively increases as $\chi \rightarrow \chi_{s}$, experiments which isothermally approach phase separation by increasing degree of polymerization may detect apparent $N$-scaling laws for $D$ with effective exponents larger than the pure homopolymer value.

Model calculations of $J_{\mathrm{A}}(\phi)=J_{\mathrm{B}}(1-\phi)$ as a function of blend composition at fixed thermodynamic state along the spinodal and binodal curves, and also at fixed values of positive and negative values of $\chi N$ corresponding to a constant temperature experiment, are shown in Fig. 30. The vertical scale is not absolute; estimates of the required nonuniversal prefactors suggest the maximum enhancement of the entanglement friction due to concentration fluctuations may be quite modest ${ }^{45)}$. This 
Fig. 30. Concentration-fluctuation friction contribution for species A as a function of its volume fraction at the spinodal, binodal, and three fixed values of $\chi N$. The vertical scale is not in absolute units

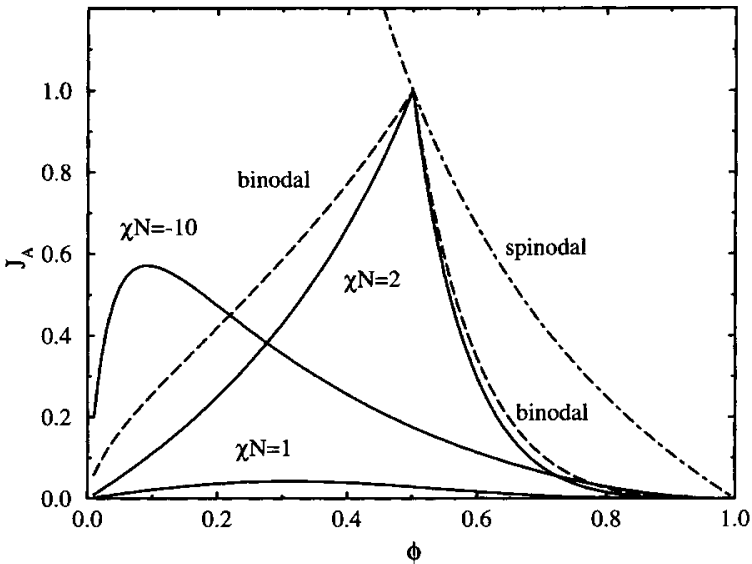

conclusion also implies negligible effects for unentangled blends may be common, in apparent accord with experimental ${ }^{116)}$ and computer simulation ${ }^{17)}$ studies of short chain blends near a critical point.

There are several other notable trends deducable from Fig. 30. (i) For experiments carried out at the critical point $(\chi N=2)$, the reduced diffusion constant $D / D_{0}$ is a minimum at the critical composition, and is an asymmetrically concave upward function of $\phi$. (ii) Even at fixed thermodynamic state the reduction of $D$ by concentration fluctuations still decreases upon moving away from the critical composition. (iii) As expected based on the local clustering asymmetry in Eq. (8.7), suppression of the minority species diffusion constant by concentration fluctuations is larger than for the majority species. (iv) Diffusion suppression turns on rapidly as the phase boundary is approached, e.g., $J$ increases by roughly an order of magnitude as $\chi N$ increases from 1 to 2 . (v) Substantial diffusion constant suppression is possible in negative $\chi$, miscible or "specific interaction" mixtures. For the idealized symmetric model considered, all the above trends also apply to the conformational relaxation time and single polymer contribution to the stress.

Self-diffusion experiments in entangled blends are typically carried out on chemically complex materials characterized by strong structural asymmetries, composition-dependent bare monomeric friction constants and glass transition temperatures, and asymmetries in degree of polymerization ${ }^{107,108)}$. These features make direct comparisons with the theoretical predictions difficult, although some observations concerning the composition and $N$-dependence of $D$ in blends are in qualitative accord with the predicted theoretical trends ${ }^{45}$. Experiments employing isotopic blends would be the optimum systems to test the theoretical predictions not only because of their chemical simplicity, but their very low value of $\chi$ would allow the most heavily entangled miscible mixtures to be studied. Computer simulation of entangled, symmetric binary blends close to the critical point would also be very valuable for testing the PMC theory. 


\section{Analytic predictions, model calculations, and comparison with experiments for symmetric diblock copolymers}

For diblock copolymers near and below the ODT, the scattering function $S_{\phi}(k)$ is strongly peaked at $S^{*}=S_{\phi}\left(k^{*}\right)$, thereby allowing a dominant one-wavevector approximation $^{113,114)} S_{\phi}(k) \sim\left(S^{*}\right)^{1 / 2} \delta\left(k-k^{*}\right)$, to be employed for semiquantitative analysis. Based on this, and Eqs. (8.4), the key factor $J \sim\left[\Delta g(\sigma) / \xi_{\rho}\right]^{2}(S * N)^{1 / 2}$. Analytic scaling law predictions based on PMC theory then follow from the known dependence of $S^{*}$ and $\Delta g$ on $N$ and thermodynamic state variables. The general equilibrium calculations based on PRISM theory with fluctuation corrections, analytic results for limiting cases, and determination of an effective ODT temperature, $T_{\mathrm{ODT}}$, are discussed elsewhere ${ }^{114,115)}$. A representative example of theory/small angle scattering experiment comparison ${ }^{15)}$ is shown in Fig. 31. Analytic analysis yields the results ${ }^{114)}: S^{*} \sim N\left(T \gg T_{\mathrm{ODT}}\right), S^{*} \sim N^{4 / 3}\left(T=T_{\mathrm{ODT}}\right)$, and $S^{*} \sim N^{2}\left(T \ll T_{\mathrm{ODT}}\right)$. The latter fluctuation-saturation, or low $T$ ground state, prediction is a novel consequence of PRISM theory and its enthalpically-driven feedback mechanism for concentration-fluctuation stabilization of a globally isotropic low temperature phase ${ }^{114)}$. The local clustering function is given by ${ }^{44,46)}$

$$
\begin{aligned}
\Delta g & =g_{\mathrm{AA}}+g_{\mathrm{BB}}-2 g_{\mathrm{AB}} \\
& \cong \frac{3}{\pi \rho \sigma^{3} \sqrt{N}}\left[12 \sqrt{\frac{S^{*}}{N}}-\sqrt{\frac{3 N}{S^{*}}}+\sqrt{6 \chi_{\mathrm{s}} N}-\frac{12}{\sqrt{2 \chi_{\mathrm{s}} N}}\right]
\end{aligned}
$$

which properly vanishes as $\chi \rightarrow 0$, and where $\chi=\chi_{\mathrm{s}}-\left(2 S^{*}\right)^{-1}$ with $\chi_{\mathrm{s}}$ equal to the mean field critical value ( 10.495 for $f=1 / 2$ ). Combining these results yields: $\Delta g \sim$ $N^{-1 / 2}\left(T \gg T_{\mathrm{ODT}}\right)$ in agreement with the blend case, $\Delta g \sim N^{-1 / 3}\left(T=T_{\mathrm{ODT}}\right)$, and $\Delta g$ $\sim N^{0}\left(T \ll T_{\mathrm{ODT}}\right)$. The low temperature "intrinsic" result is physically sensible for a highly segregated liquid composed of nearly pure A and B microdomains. The cor-

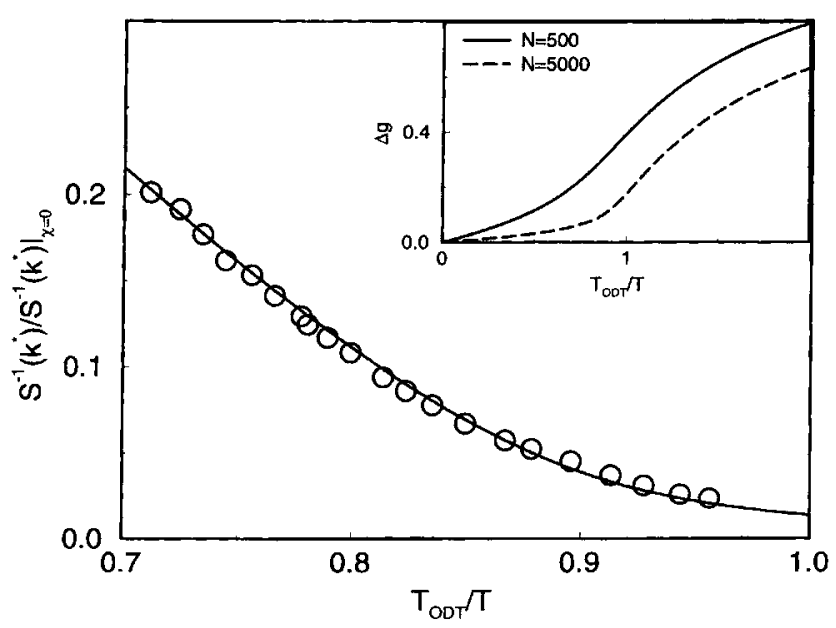

Fig. 31. Best fit ${ }^{115)}$ $\left(\xi_{\rho} / \sigma=0.14\right)$ of PRISM theory (full line) to experimental inverse peak scattering intensity as a function of reduced inverse temperature for a symmetric PEPPEE (polyethylenepropylene-polyethylethylene) polyolefin melt of $N=791$. The inset shows PRISM model calculations for the local clustering function for $f=$ $0.5, \xi_{p} / \sigma=0.3$ 
responding frictional enhancement factor $J$ grows as the diblock is cooled and obeys the qualitative power laws: $J \sim(S * / N)^{3 / 2} \sim N^{0}\left(T \gg T_{\mathrm{ODT}}\right), N^{1 / 2}\left(T=T_{\mathrm{ODT}}\right)$, and $N^{3 / 2}$ ( $T \ll T_{\text {ODT }}$ ). Thus, the entangled homopolymer and blend law of $D \sim N^{-2}$ is predicted far from the ODT. However, new scaling laws emerge at and below the ODT: $D \sim N^{-5 / 2}$ at $T=T_{\mathrm{ODT}}$, and $D \sim N^{-7 / 2}$ for $T \ll T_{\mathrm{ODT}}$.

Following Lodge and coworkers ${ }^{105,106)}$, the ratio $D / D_{0}$ in Eq. (8.5) is employed to quantify diffusion suppression by microdomain formation. For viscoelastic or dielectric relaxation experiments, the relevant conformational relaxation time ratio is $\tau / \tau_{0} \cong\left(D_{0} / D\right)\left(R_{\mathrm{g}} / R_{\mathrm{g}, 0}\right)^{2}$. The fundamental prediction of PMC theory based on PRISM input is the existence of three distinct dynamical regimes ${ }^{46)}$. (1) Far from the ODT the concentration fluctuations have little or no effect, i.e., $D / D_{0} \cong 1$ and nearly $T$-independent. This regime is qualitatively similiar to the entangled blend case. (2) As the ODT is approached, and somewhat below it, $D / D_{0}$ decreases rapidly due to enhanced physical clustering and microdomain formation, and a strong thermal dependence of diffusion suppression emerges. The precise magnitude and form of $D / D_{0}$ as a function of reduced temperature $T_{\mathrm{ODT}} / T$ is not expected to be universal since it is influenced by system-specific equilibrium structure and entanglement characteristics of the blocks. (3) Well below the ODT, $D / D_{0}$ approaches its minimum (nonuniversal) value proportional to $N^{-3 / 2}$, which is $T$-independent due to the saturation of concentration fluctuations and microdomain coarsening at low $T$.

The predicted three regime scenario described above is in excellent qualitative accord with recent experiments on lamallae-forming diblock melts ${ }^{106)}$. It also seems at least consistent with the "thermally activated" (reptative) and $T$-independent "entropic" (arm-retraction) interpretation of Lodge and coworkers ${ }^{106)}$. Of course, PMC theory focuses on the ensemble-averaged friction, and not the precise nature of copolymer trajectories, which severely limits the deduction of an intuitive real space picture. Within PMC theory the fundamental physical effect is the thermodynamic driving force to keep the A and B segments segregated prolongs relaxation of the entanglement friction forces, thereby slowing down copolymer diffusion and conformational relaxation.

Model calculations of $J$ and $D / D_{0}$ which exhibit the three limiting regimes, and crossovers between them, are shown in Fig. 32. A melt-like screening length of $\xi_{\rho} / \sigma$ $=0.3$ and a numerical prefactor of $\lambda=0.07$ (see below) were employed. At intermediate temperatures the reduced diffusion constant decreases in an approximately exponential manner, consistent with the "thermally-activated" interpretation ${ }^{106)}$.

The PMC theory has also been quantitatively applied by Guenza, Tang and Schweizer ${ }^{44,46)}$ to several tracer and self-diffusion experiments of Lodge and coworkers $^{105,106)}$. Consider first the self-diffusion case for four well entangled, $f \cong 1 / 2$ PEP-PEE (polyethylenepropylene-polyethylethylene) polyolefin melts. The first comparison is for two quenched (globally isotropic) materials ${ }^{105}$ ): PEP-PEE1 $(N=$ 562 based on $\left.M_{\text {segment }}=56 \mathrm{~g} / \mathrm{mol}\right)$ and PEP-PEE $2(N=895)$. PEP-PEE 1 is far above its ODT of $-41^{\circ} \mathrm{C}$, and thus presumed to obey simple homopolymer dynamics unaffected by long range concentration fluctuations. PEP-PEE2 has a $T_{\mathrm{ODT}}=96^{\circ} \mathrm{C}$. The data is shown in the top panel of Fig. 33, along with an estimate of what $D$ for PEPPEE2 would be if concentration fluctuation effects were negligible. The latter esti- 


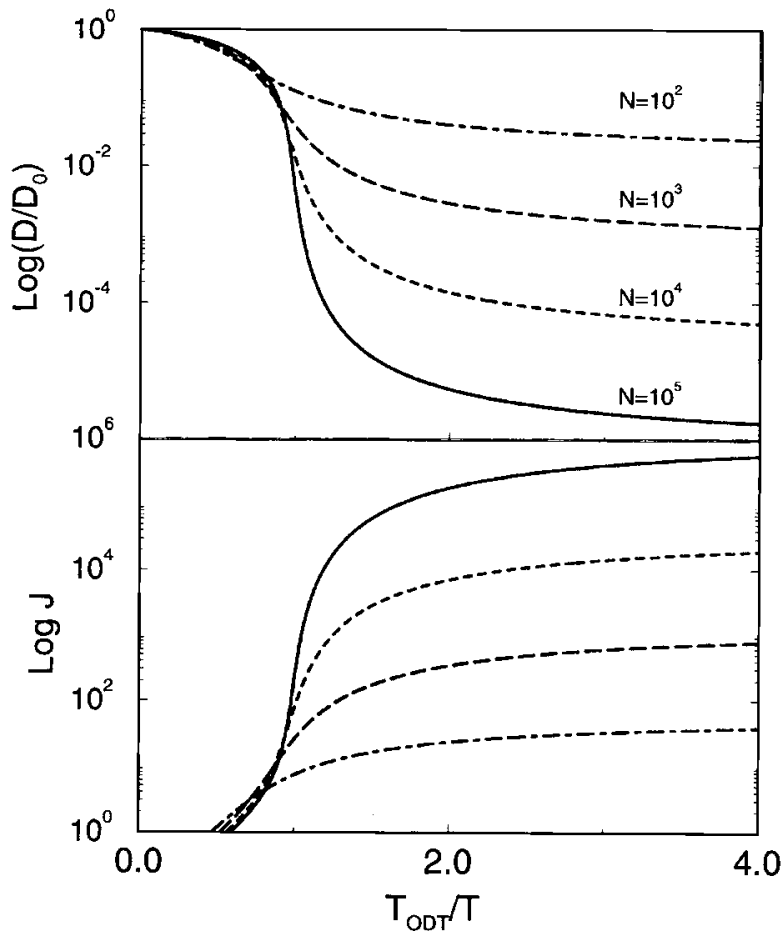

Fig. 32. Log-linear plot of the normalized diffusion constant and the factor $J$ as a function of normalized inverse temperature for $f=0.5$, $\xi_{\rho} / \sigma=0.3, \lambda=0.07$, and several values of $N$

mate falls above the data, and increasingly so (up to nearly a factor of 10) as the material becomes more segregated. A best fit of the theory to the PEP-PEE2 data is also shown, where the numerical factor $\lambda$ and screening length were varied. The resultant value of $\xi_{\rho} / \sigma=0.3$ is sensible for a melt, and the predicted temperature dependence is in excellent agreement with the data ${ }^{105}$.

A much more severe test of the theory is the most recent experimental data for shear oriented PEP-PEE materials ${ }^{106}$. This study included PEP-PEE2 plus two higher molecular weight samples, PEP-PEE3 $(N=1450)$ and PEP-PEE4 $(N=$ 1970). The "parallel" self-diffusion constant data versus reduced temperature is shown in the lower panel of Fig. 33. The measured anisotropy of the diffusion constants were surprisingly small for these lamallae forming systems, and is ignored in our PMC theory based on isotropic dynamics. PMC predictions are also shown based on exactly the same values of $\lambda$ and $\xi_{\rho} / \sigma$ extracted from the analysis of quenched PEP-PEE2 data. We emphasize these results are not a fit. The agreement of experiment and theory is very good ${ }^{46)}$.

Recent experiments have also probed entangled tracer PEP-PEE diffusion in the highly segregated $\left(T_{\mathrm{ODT}} \cong 500^{\circ} \mathrm{C}\right)$ PEP-PEE4 matrix ${ }^{106)}$. The corresponding tracer values of $D / D_{0}$ were found to be temperature-independent, and $\left(D / D_{0}\right)_{\mathrm{tr}} \cong 0.012 \pm$ 0.004 (PEP-PEE4), $0.020 \pm 0.05$ (PEP-PEE2), and $0.6 \pm 0.1$ (PEP-PEE1). Although quantitative generalization of PMC theory for tracer diffusion is technically involved $^{110)}$, semiquantitative analysis is very simple ${ }^{46)}$. Since the $T$-dependence of 
Fig. 33. Top panel: Comparison of the best fit theory (full lines) and experimental data ${ }^{105}$ ) for the quenched sample of PEP-PEE2 discussed in the text. Data for PEP-PEE1, and the estimated entangled result in the absence of concentration fluctuations (dashed curve), are also shown. Bottom panel: Comparison of theory (with same $\lambda$ and $\xi_{\rho} / \sigma$ values as in top panel) with the normalized parallel diffusion constant data of the shear-aligned PEP-PEE samples ${ }^{106)}$ discussed in the text
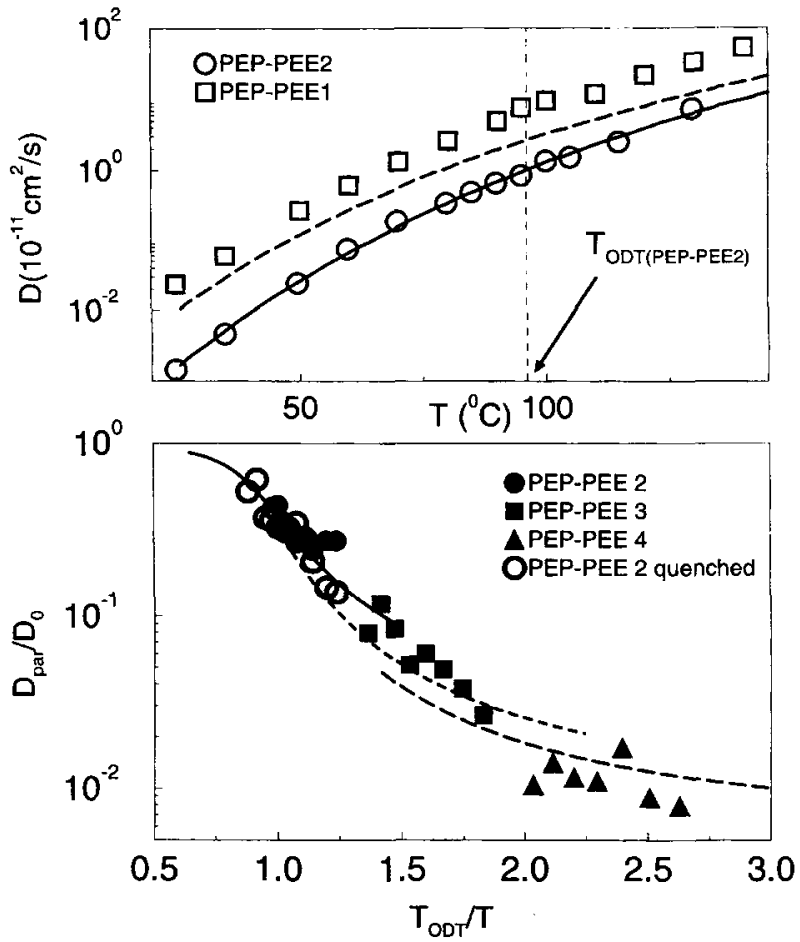

$D / D_{0}$ arises solely from the matrix concentration fluctuations, $S^{*}$, one can immediately conclude $T$-independence is predicted for $D / D_{0}$ in PEP-PEE4 where $T \ll T_{\text {ODT }}$. Moreover, for entangled diblock tracers shorter $\left(N_{t}\right)$ than the chemically and compositionally identical matrix diblocks $(N)$, the qualitative dependence of $D / D_{0}$ on tracer degree of polymerization follows from a simple power counting analysis of the known dependence on $N_{\mathrm{t}}$ of $\Delta g(\sigma)$ and $\omega(k)$ in Eq. (8.4). The result is $J \sim\left(N_{\mathrm{t}} / N\right)^{7 / 2}$. Equivalently, the reduced tracer diffusion constant can be expressed in terms of the corresponding self-diffusion ratio as ${ }^{46)}$

$$
\left(\frac{D}{D_{0}}\right)_{\mathrm{tr}} \cong\left(1+\left[\left(D / D_{0}\right)_{\mathrm{self}}-1\right]\left(\frac{N_{\mathrm{t}}}{N}\right)^{7 / 2}\right)^{-1}
$$

Thus, based on this result and the experimental value of $\left(D_{0} / D\right)_{\text {self }}=90 \pm 30$ for PEP-PEE4, one obtains the predictions: $\left(D / D_{0}\right)_{\mathrm{tr}} \cong 0.17 \pm 0.05$ (PEP-PEE2) and $(D /$ $\left.D_{0}\right)_{\mathrm{tr}} \cong 0.5 \pm 0.1$ (PEP-PEE1), which are in remarkable agreement with the experimental values quoted above. For a PEP-PEE3 tracer, not studied experimentally, the theory predicts $\left(D / D_{0}\right)_{\mathrm{tr}} \cong 0.035 \pm 0.012$. Physically, the reduced diffusion constant suppression for shorter tracers is due to the smaller frictional penalty required to dynamically transport $A(B)$ tracer segments through the $B$ (A) matrix microdomains. 
The PMC theory of diblock copolymer diffusion can be extended to treat the consequences of copolymer composition [anisotropic diffusion is of far less concern (irrelevant) for cylindrical (spherical) microphases], block bare friction constant asymmetry, chain stretching, dilution by neutral solvent, and matrix constraint release $^{110)}$. Homopolymer and diblock tracer diffusion can also be addressed $44,46,110)$. The full PMC theory can be generalized to finite frequen$\operatorname{cies}^{109,110)}$, thereby allowing treatment of concentration fluctuation effects on early time (anomalous) diffusion, the unentangled-entangled crossover, and internal normal mode dynamics and shear stress moduli. All these extensions are presently underway. We do caution, however, that the cage-averaged isotropic dynamics approach is expected to eventually fail, perhaps at very high degrees of segregation and/or for situations of strongly spatially-dependent local friction.

\section{Future directions and open problems}

This article has summarized the significant progress made over the last eight years in formulating and applying the first microscopic liquid-state theory of entangled polymer dynamics in solutions, melts, gels, and multicomponent materials. Recent theoretical generalizations, and detailed applications to experiments, in areas such as (i) finite $N$ corrections to transport coefficients and frequency-dependent response functions in linear chain solutions, melts and gels ${ }^{40,41,48,77)}$, (ii) fractal macromolecular architectures ${ }^{47)}$, and (iii) self and tracer diffusion in polymer blends and diblock copolymers ${ }^{44-46)}$, are particularly noteworthy. These novel developments establish the power and promise of the PMC approach for: (i) providing a microscopic understanding of long-standing experimental puzzles, (ii) establishing universal and system-specific links between the entanglement forces, polymer and liquid structure, and intermediate and long time dynamics, (iii) for making new predictions testable by experiment and/or simulation.

Treatment of a number of new systems and phenomena seem within reach in the near future based on extensions of the existing PMC theory. These include: (i) analytic and numerical finite $N$ corrections for entanglement dynamics of non-chain architectures, (ii) tracer diffusion in mixed systems of chains, rings, and/or microgels ${ }^{118)}$, (iii) chain dynamics, and friction constant asymmetry and collective constraint release effects, in multicomponent entangled fluids, and (iv) the influence of polydispersity on dynamics. In the somewhat longer term, progress based on the PMC approach, possibly in conjunction with field theoretic and/or other statistical dynamical methods, should be possible for problems such as: anisotropic diffusion in block copolymer microphases, entangled alloy collective rheological response, local and glassy polymer dynamics, mixtures of colloids and polymers, semidilute and concentrated polyelectrolyte solutions including excluded volume and dielectric friction, associating entangled polymer fluids such as ionomers and hydrogenbonded materials, and hydrodynamic effects in solution.

On the other hand, there remain many systems and physical phenomena which may require fundamental theoretical advances and/or significant modifications of the existing PMC approach. Open problems in this category include: (i) entangle- 
ment dynamics of rigid rods, branched polymers (with their exponentially slow dynamics), non-fractal microgels or "rubber balls", and dendrimers, (ii) the influence of quenched physical or chemical disorder, (iii) spatially resolved, nonlinear time-correlation functions such as the incoherent and coherent single polymer dynamic structure factors, and (iv) nonlinear rheological phenomena. Technical simplifications of the present effective-medium-like PMC theory which may require strong modification in order to treat some of these problems include: the isotropic cage-averaged description, static and dynamic equivalent site approximation, neglect of explicitly anisotropic motions such as reptation and arm retraction, an effective linear GLE approach which retains the integrity of Rouse modes, and neglect of strong coupling "self-consistent" feedback effects at the level of the memory functions. We remain optimistic that progress can be made on these difficult theoretical issues.

Finally, a more fundamental understanding of entangled dynamics would emerge from a theory formulated at the level of two interacting chains. Such 2-chain dynamical information is not explicitly contained in the present PMC approach, nor the Rouse or reptation/tube theories, which all represent single chain "mean-field-like" approaches. The latter level of description is entirely adequate for predicting all dynamical properties of a single macromolecule dissolved in a condensed phase. However, knowledge of the effective two chain dynamics in the liquid is required to address questions such as the collective intermolecular contributions to stress, dielectric relaxation, and other dynamical response functions in solutions, melts and multicomponent polymer alloys. For simple atomic, colloidal and molecular fluids, or any liquid near a critical point, such collective contributions are well known to be of central importance.

Formally, the statistical dynamical approach described here and in the original PMC paper ${ }^{36)}$ can be generalized to derive nonlinear GLE's for two tagged polymers in a condensed phase. Results of the form of Eqs. (2.1) and (2.2) are obtained, but with two essential modifications ${ }^{119)}$. First, the time-delayed memory function contributions for each chain involve the positions of all the segments of both tagged macromolecules. Second, the 2-chain potential of mean force contains the standard intramolecular (Gaussian spring) part, plus an effective interchain pair interaction given by $-k T \ln \{g(r)\}$. Due to the universal correlation hole effect, the latter contains a spatially long range contribution of a screened Coulomb form, and hence is of a highly nonlinear mathematical form. Physically, the existence of Coulombic-like forces on intermediate length scales $\left(\sigma \ll r<R_{\mathrm{g}}\right.$ ) between all pairs of segments on the two tagged polymers is expected to induce strong spatial correlations of their relative dynamical motions ${ }^{119}$ ). The development of a tractable microscopic theory at the two chain level which simultaneously takes into account both the long range interchain potential of mean force, and the non-Markovian, nonlocal influences of the surrounding matrix polymers on the two tagged chains, remains a challenging theoretical task. 
Acknowledgement: Research at Illinois was supported by the Department of Energy, Basic Energy Science Division in cooperation with Martin Marietta Corporation and Oak Ridge National Laboratories, and the UIUC-MRL DOE-BES contract DEFG0291ER45439 and DEFG02-96ER45439. DOE support from Sandia National Laboratory via CRADA \#1078, and the U.S. National Science Foundation via the UIUC-MRL grant NSF-DMR-89-20538, are also acknowledged. Partial support for M.F. was from the Deutsche Forschungsgemeinschaft under grant number Fu309/1-1. M.G. thanks the $I M A G-C N R$ of Italy for the opportunity to carry out this research at Illinois.

1) J. D. Ferry, "Viscoelastic Properties of Polymers", Wiley, New York 1980

2) P. G. deGennes, "Scaling Concepts in Polymer Physics", Cornell U. Press 1979

3) M. Doi, S. F. Edwards, "The Theory of Polymer Dynamics", Oxford University Press, Oxford 1986

4) W. W. Graessley, Adv. Polym. Sci. 47, 68 (1982); D. Pearson, Rubber Chem. Technol. 60, 437 (1987)

5) T. P. Lodge, N. Rotstein, S. Prager, Adv. Chem. Phys. 79, 1 (1990); A. L. Kholodenko, Macromol. Theory Simul. 5, 1031 (1996)

6) J. Roovers, Macromolecules 21, 1517 (1988); G. B. McKenna, B. J. Hofstetter, N. Hadjichristidis, L. J. Fetters, D. J. Plazek, Macromolecules 22, 1834 (1989)

7) W. W. Graessley, J. Roovers, Macromolecules 12, 959 (1984); L. J. Fetters, A. D. Kiss, D. S. Pearson, G. F. Quack, F. J. Vitus, Macromolecules 26, 647 (1993); K. Shull, E. J. Kramer, L. J. Fetters, Nature 345, 790 (1990)

8) J. Roovers, Macromolecules 17, 1196 (1984); J. Roovers, P. M. Toporowski, Macromolecules 20, 2300 (1987)

9) M. Antonietti, T. Pakula, W. Bremser, Macromolecules 28, 4227 (1995)

10) "Polyelectrolytes", M. Hara, Ed., Marcel Dekker, New York 1993

11) L. A. Utracki, "Polymer Alloys Blends: Thermodynamics Rheology", Hanser Publishers, Munich 1989

12) G. H. Fredrickson, F. S. Bates, Annu. Rev. Mater. Sci. 26, 503 (1996); R. H. Colby, Curr. Opin. Coll. Inter. Sci. 1, 454 (1996)

13) R. A. Register, R. K. Prud'homme, in "Ionomers: Synthesis, Structure, Properties and Applications", Chapman \& Hall, New York 1996

14) J. Skolnick, A. Kolinski, Adv. Chem. Phys. 78, 223 (1990)

15) G. Ronca, J. Chem. Phys. 79, 1031 (1983)

16) M. Fixman, J. Chem. Phys. 89, 3892 (1988)

17) M. F. Herman, J. Chem. Phys. 92, 2043 (1990); M. F. Herman, B. Panajotova, K. T. Lorenz, J. Chem. Phys. 105, 1153 (1996), and refs. cited therein

18) J. F. Douglas, J. B. Hubbard, Macromolecules 24, 3163 (1991)

19) A. P. Chatterjee, R. F. Loring, J. Chem. Phys. 103, 4711 (1995)

20) K. Adachi, T. Wada, T. Kawamoto, T. Kotaka, Macromolecules 28, 3588 (1995); B. T. Poh, K. Adachi, T. Kotaka, Macromolecules 29, 6317 (1996), and refs. cited therein; K. Adachi, H. Yoshida, F. Fukai, T. Kotaka, Macromolecules 23, 3138 (1990)

21) N. Nemoto, in "Polymer Rheology, Processing", London, New York, A. Collyer, L. Utracki, Eds., Elsevier LTD, UK 1990, p. 3; N. A. Rotstein, T. P. Lodge, Macromolecules 25, 1316 (1992)

22) J. Jackson, M. E. Rosa, H. H. Winter, Macromolecules 27, 2426 (1994); H. H. Winter, J. Noncryst. Sol. 172-174, 1158 (1994)

23) M. Doi, J. Polym. Sci., Polym. Phys. Ed. 21, 667 (1983)

24) M. Rubinstein, Phys. Rev. Lett. 591946 (1987) 
25) J. des Cloizeaux, Europhys. Lett. 5, 437 (1988)

26) G. Marruci, J. Polym. Sci., Polym. Phys. Ed. 23, 159 (1985)

27) H. Watanable, M. Tirrell, Macromolecules 22, 927 (1989); M. Doi, D. Pearson, J. Kornfield, G. Fuller, Macromolecules 22, 1488 (1989)

28) W. Hess, Macromolecules 19, 1395 (1986); 20, 2587 (1987); 21, 2620 (1988)

29) K. Kawasaki, Mod. Phys. Lett. B 4, 913 (1990)

30) V. G. Rostiashvili, Sov. Phys. JETP 70, 563 (1990)

31) K. Binder, W. Paul, J. Polym. Sci., Part B: Polym. Phys. 35, 1 (1997); K. Kremer, G. S. Grest, J. Chem. Phys. 92, 5057 (1990); J. Chem. Soc., Faraday Trans. 88, 1707 (1992)

32) C. M. Ylitalo, G. G. Fuller, V. Abetz, R. Stadler, D. Pearson, Rheol. Acta 29, 543 (1990), refs. cited therein

33) G. Agrawal, R. P. Wool, W. D. Dozier, G. P. Felcher, T. P. Russell, J. W. Mays, Macromolecules 27, 4407 (1994); J. Polym. Sci., Part B: Polym. Phys. 34, 2916 (1996)

34) C. J. Grayce, G. Szamel, K. S. Schweizer, J. Chem. Phys. 102, 2222 (1995)

${ }^{35)}$ D. E. Smith, T. T. Perkins, S. Chu, Phys. Rev. Lett. 75, 4146 (1995)

36) K. S. Schweizer, J. Chem. Phys. 91, 5802 (1989)

37) K. S. Schweizer, J. Chem. Phys. 91, 5822 (1989)

38) K. S. Schweizer, J. Non-Cryst. Solids, 131-133, 643 (1991)

39) K. S. Schweizer, Phys. Scripta T49, 99 (1993)

40) K. S. Schweizer, G. Szamel, Phil. Mag. B 71, 783 (1995)

41) K. S. Schweizer, G. Szamel, Trans. Theor. Stat. Phys. 24, 947 (1995)

42) K. S. Schweizer, G. Szamel, J. Chem. Phys. 103, 1934 (1995)

43) K. S. Schweizer, G. Szamel, Macromolecules 28, 7543 (1995)

44) H. Tang, K. S. Schweizer, J. Chem. Phys. 103, 6296 (1995)

45) H. Tang, K. S. Schweizer, J. Chem. Phys. 105, 779 (1996)

46) M. Guenza, H. Tang, K. S. Schweizer, Macromolecules 30, 3423 (1997)

47) M. Fuchs, K. S. Schweizer, J. Chem. Phys. 106, 347 (1997)

48) M. Fuchs, K. S. Schweizer, Macromolecules, in press

49) K. S. Schweizer, J. G. Curro, Phys. Rev. Lett. 58, 246 (1987)

50) J. P. Hansen, I. R. McDonald, "Theory of Simple Liquids", $2^{\text {nd }}$ edition, Academic, London 1986; J. P. Boon, S. Yip, "Molecular Hydrodynamics", McGraw-Hill, New York 1980

51) W. Hess, R. Klein, Adv. Phys. 32, 173 (1983)

52) W. Götze, L. Sjögren, Rep. Prog. Phys. 55, 241 (1992)

53) K. Kawasaki, Ann. Phys. 61, 1 (1970)

54) P. C. Hohenberg, B. Halperin, Rev. Mod. Phys. 49, 435 (1977)

55) S. Havlin, D. BenAvraham, Adv. Phys. 36, 695, and refs. cited therein; T. Nakayama, K. Yakubo, R. Orbach, Rev. Mod. Phys. 66, 381 (1994)

56) M. Guenza, K. F. Freed, J. Chem. Phys. 105, 3823 (1996), and refs. therein

57) For recent reviews see: K. S. Schweizer, J. G. Curro, Adv. Polym. Sci. 116, 319 (1994); Adv. Chem. Phys. 98, 1 (1997)

58) D. Chandler, H. C. Andersen, J. Chem. Phys. 57, 1930 (1972)

59) K. S. Schweizer, E. F. David, C. Singh, J. G. Curro, J. J. Rajasekaran, Macromolecules 28, 1528 (1995)

60) K. S. Schweizer, J. G. Curro, Chem. Phys. 149, 105 (1990)

61) M. Bixon, J. Chem. Phys. 58, 1459 (1973); R. Zwanzig, J. Chem. Phys. 60, 2717 (1974); M. Bixon, R. Zwanzig, J. Chem. Phys. 68, 1896 (1978)

62) For a discussion of chemical dynamics problems see: G. Goodyear, R. M. Stratt, J. Chem. Phys. 105, 10050 (1996), and refs. cited therein; J. M. Ziman, "Models of Disorder", Cambridge Press, London 1979

63) A. Z. Akcasu, M. Benmouna, C. C. Han, Polymer 21, 866 (1980)

64) N. Fatkullin, R. Kimmich, J. Chem. Phys. 101, 822 (1994) 
${ }^{65)}$ L. J. Fetters, D. J. Lohse, D. Richter, T. A. Witten, A. Zirkel, Macromolecules 27, 4639 (1994)

${ }^{66)}$ R. Cerf, J. Phys. Radium 19, 122 (1958); V. N. Pokrovskii, Sov. Phys. Usp. 35, 384 (1992); V. N. Pokrovskii, V. S. Volkov, Polym. Sci. USSR 20, 3029 (1979); A. I. Leonov, J. Rheol. 38, 1 (1994); D. A. McInnes, A. M. North, Polymer 18, 505 (1977)

${ }^{67)}$ R. Verbeg, I. M. deSchepper, E. G. D. Cohen, Phys. Rev. E 55, 3143 (1997)

${ }^{68)}$ A. N. Semenov, Physica A 166, 263 (1990); U. Genz, Macromolecules 27, 3501 (1994)

69) P. G. deGennes, J. Phys. (Paris) 42, 735 (1981); J. des Cloizeaux, J. Phys. I (France) 3,1523 (1993)

${ }^{70)}$ E. Raspaud, D. Lairez, M. Adam, Macromolecules 28, 927 (1995)

71) N. P. T. O'Connor, R. C. Ball, Macromolecules 25, 5677 (1992)

72) J. des Cloizeaux, Macromolecules 23, 4678 (1990); 25, 835 (1992)

${ }^{73)}$ D. Richter, B. Farago, R. Butera, L. J. Fetters, J. S. Huang, B. Ewen, Macromolecules 26, 795 (1993); D. Richter, L. Willner, A. Zirkel, B. Farago, L. J. Fetters, J. S. Huang, Macromolecules 27, 7437 (1994)

${ }^{74)}$ M. Appel, G. Fleischer, J. Karger, F. Fujara, I. Chang, Macromolecules 27, 4274 (1994), and refs. cited therein

75) N. Fatkullin, R. Kimmich, Phys. Rev. E 52, 3273 (1995)

76) A. P. Chatterjee, R. F. Loring, J. Chem. Phys. 101, 1595 (1994)

77) G. Szamel, K. S. Schweizer, in preparation

${ }^{78)}$ W. Paul, K. Binder, D. W. Heermann, K. Kremer, J. Phys. II (Paris) 1, 37 (1991); J. Chem. Phys. 95, 7726 (1991)

79) Y. Termonia, Macromolecules 29, 2025 (1996)

${ }^{80)}$ P. F. Green, E. J. Kramer, Macromolecules 19, 1108 (1986)

${ }^{81)}$ N. Nemoto, M. Kishine, T. Inoue, K. Osaki, Macromolecules 24, 1648 (1991); 23, 659 (1990); 22, 3793 (1989)

${ }^{82)}$ M. Muthukumar, A. Baumgärtner, Macromolecules 22, 1937, 1941 (1989)

${ }^{83)}$ M. Muthukumar, A. Baumgärtner, Adv. Chem. Phys. 94, 625 (1996)

${ }^{84)}$ M. Rubinstein, S. P. Obukhov, Phys. Rev. Lett. 71, 1856 (1993)

${ }^{85)}$ J. Klein, Macromolecules 11, 852 (1978); 19, 105 (1986)

${ }^{86)}$ R. H. Colby, L. J. Fetters, W. W. Graessley, Macromolecules 20, 2226 (1987)

87) J. Roovers, Polymer 26, 1091 (1985)

${ }^{88)}$ D. S. Pearson, L. J. Fetters, W. W. Graessley, G. V. Strate, E. von Meerwall, Macromolecules 27, 711 (1994)

89) G. Fleischer, M. Appel, Macromolecules 28, 7281 (1995)

${ }^{90)}$ M. Appel, G. Fleischer, Macromolecules 25, 5520 (1993)

91) M. Antonietti, K. J. Folsch, H. Sillescu, Makromol. Chem. 188, 2317 (1987)

${ }^{92)}$ A. T. Andrews, "Electrophoresis: Theory, Techniques, and Biochemical, and Clinical Applications", Oxford University Press, Oxford 1986

${ }^{93)}$ F. Horkay, A. M. Hecht, E. Geissler, Faraday Disc. 101, 159 (1995)

${ }^{94)}$ F. Ferri, B. J. Frisken, D. S. Cannell, Phys. Rev. Lett. 67, 3626 (1991)

${ }^{95)}$ N. A. Rotstein, T. P. Lodge, Macromolecules 25, 1316 (1992)

${ }^{96)}$ G. W. Slater, S. Y. Wu, Phys. Rev. Lett. 75, 164 (1995)

97) B. Zimm, O. Lumpkin, Macromolecules 26, 226 (1993)

${ }^{98)}$ E. Arvanitidou, D. Hoagland, Phys. Rev. Lett. 67, 1464 (1991)

${ }^{99)}$ M. Muthukumar, J. Chem. Phys. 83, 3161 (1985)

${ }_{100)}$ M. E. Cates, Phys. Rev. Lett. 53, 926 (1984); J. Phys. (Paris) 46, 1059 (1985)

${ }^{101)}$ M. E. Cates, J. M. Deutsch, J. Phys. (Paris) 47, 2121 (1986)

${ }^{102)}$ M. Müller, J. P. Wittmer, M. E. Cates, Phys. Rev. E 53, 5063 (1996)

${ }^{103)}$ G. Szamel, Phys. Rev. Lett. 70, 3744 (1993); G. Szamel, K. S. Schweizer, J. Chem. Phys. 100, 3127 (1994) 
${ }^{104)}$ K. R. Shull, E. J. Kramer, F. S. Bates, J. H. Rosedale, Macromolecules 24, 1383 (1991)

${ }^{105)}$ M. C. Dalvi, C. E. Eastman, T. P. Lodge, Phys. Rev. Lett. 71, 2591 (1993); M. C. Dalvi, T. P. Lodge, Macromolecules 27, 3487 (1994); C. E. Eastman, T. P. Lodge, Macromolecules 27, 5591 (1994)

106) T. P. Lodge, M. C. Dalvi, Phys. Rev. Lett. 75, 657 (1995)

${ }^{107)}$ R. J. Composto, E. J. Kramer, D. M. White, Polymer 31, 2320 (1990); E. Kim, E. J. Kramer, W. C. Wu, P. D. Garrett, Polymer 35, 5706 (1994); E. Kim, E. J. Kramer, J. O. Osby, Macromolecules 28, 1979 (1995)

${ }^{108)}$ P. F. Green, D. B. Adolf, L. R. Gilliom, Macromolecules 24, 3377 (1991); P. F. Green, Macromolecules 24, 3373 (1991)

109) U. Genz, T. A. Vilgis, J. Chem. Phys. 101, 7101, 7111 (1994)

${ }^{110)}$ M. Guenza, K. S. Schweizer, J. Chem. Phys., submitted

${ }^{111)}$ K. S. Schweizer, A. Yethiraj, J. Chem. Phys. 98, 9053 (1993); C. Singh, K. S. Schweizer, A. Yethiraj, J. Chem. Phys. 102, 2187 (1995)

${ }^{112)}$ F. S. Bates, G. H. Fredrickson, Annu. Rev. Phys. Chem. 41, 525 (1990)

${ }^{113)}$ L. Leibler, Macromolecules 13, 1602 (1980); S. A. Brazovski, Sov. Phys. JETP, 41, 85 (1975); G. H. Fredrickson, E. Helfand, J. Chem. Phys. 87, 697 (1987)

${ }^{114)}$ E. F. David, K. S. Schweizer, J. Chem. Phys. 100, 7767 (1994)

${ }^{115)}$ M. Guenza, K. S. Schweizer, J. Chem. Phys. 106, 7391 (1997)

${ }^{116)}$ G. Fleischer, Prog. Colloid. Polym. Sci. 91, 55 (1993); S. Lang, H. Sillescu, Makromol. Chem. 190, 797 (1989)

${ }^{117)}$ M. Müller, K. Binder, J. Phys. II 6, 187 (1996)

${ }^{118)}$ K. Shull, K. Dai, E. J. Kramer, L. J. Fetters, M. Antonietti, H. Sillescu, Macromolecules 24, 505 (1991); S. F. Tead, E. J. Kramer, G. Hadziioannou, M. Antonietti, H. Sillescu, Macromolecules 25, 3942 (1992)

${ }^{119)}$ M. Guenza, K. S. Schweizer, in preparation 